TITLE:

\title{
Direct Imaging of Molecular Chains in a Poly(p-Xylylene) Single Crystal( Dissertation_全文)
}

\section{$\operatorname{AUTHOR}(S)$ :}

Tsuji, Masaki

\section{CITATION:}

Tsuji, Masaki. Direct Imaging of Molecular Chains in a Poly(p-Xylylene) Single Crystal. 京都 大学, 1981, 工学博士

\section{ISSUE DATE:}

1981-05-23

URL:

https://doi.org/10.14989/doctor.k2589

RIGHT: 
Direct Imaging of Molecular Chains

in a Poly [p-Xylylene] Single Crystal

Masaki Tsuji

1981 



\section{Direct Imaging of Molecular Chains \\ in a Poly(p-Xylylene) Single Crystal}

\section{Masaki Tsuji}

Laboratory of Polymer Crystals

Institute for Chemical Research

Kyoto University

1981 



\section{Contents}

Introduction

References

Chapter 1: Some aspects of high resolution electron microscopy 11

1-1 Scattering of electrons through the objects

1-2 Image formation in high resolution electron microscopy

1-3 Some problems in taking high resolution electron micrographs

References

Chapter 2: Morphology of PPX single crystals

2-1 Morphology of PPX single crystals

2-2 Effects of electron irradiation on PPX single crystals

References

Chapter 3: High resolution electron microscopy of $\mathrm{PPX}$ single crystals

3-1 The effect due to $500 \mathrm{KV}$ electron irradiation

3-2 Selection of the conditions for taking high resolution electron micrographs

3-3 Procedures for taking high resolution electron micrographs and the method for judgement of the fidelity of an image 
Chapter 4: Image processing for electron microscopy

4-1 Construction of image processing systems

4-2 Theoretical treatment on image processing

4-3 Optical filtering of the high resolution electron micrograph of the PPX $\beta$-form single crystal

4-4 Other examples of image processing

References

Chapter 5: Crystal structure analysis of PPX $\beta$-form crystal 106

5-1 Determination of the PPX $\beta$-form crystal structure in the projection on the ab-plane

5-2 Crystal structure analysis of the PPX $\beta$-form by $x$-ray diffraction

References

Chapter 6: Direct observation of the lattice defects in PPX single crystals

6-1 Models of dislocation in a polymer crystal

6-2 High resolution electron microscopy for observing dislocations in PPX single crystals

References

Summary

List of publications

Acknowledgments 
Introduction

"Single crystals" (in reality, mosaic crystals) of polymers 1

were first observed by Yundt on optical and electron microscopy of hemicelluloses (xylan), and subsequently by Schlesinger \& Leeper and Keller \& Waring on optical microscopy of gutta percha. In 1955 Jaccodine reported the crystallization of a low-molecular-weight linear polyethylene (PE) from dilute benzene and xylene solutions in the form of thin, spirally grown, lozenge-shaped lamellae. This work was extended to high molecular weights independently by Till, Keller, and Fischer. These authors crystallized PE single crystals from dilute solutions, and observed their morphology under the electron microscope (EM). Especially, Keller clarified that the molecular chains much longer than lamellar thickness ( $\sim 100 \AA)$ must fold back and forth. A selected-area electron diffraction pattern 
from a PE single crystal has revealed that the long chains lie mainly along the shortest crystal direction, i.e., they are normal (or at least approximately normal) to the end-surface of the lamella. As the contour length of a molecule is more than thousands of $\AA$, the molecule can not settle within a distance of $100 \AA$ or so in an extended form. The only possible alternative is the folding back of a molecule at the surface of the lamella. This suggestion had already been made as early as 1938 by storks but then it passed unheeded.

The epochal folding concept was squarely opposed to the traditional, so-called "fringed micelle" model by Herrmann et al 10 which had been a working hypothesis to explain the structure of gelatin. However, since Fischer and Kobayashi et al. independently discovered with EM that the melt-grown spherulites which had been regarded to have the fringed micelle structure were also composed of single-crystal-like lamellae, the lamellar crystal with

chain-folding has been considered as the basic structural constituent of crystalline polymer solids.

After the discovery of PE single crystal, single crystals of various polymers have been studied with 13.14 However, the application of EM was limited to morphological investigation of polymer crystals and the molecular arrangement in a crystal has never been observed with EM, not only because EM did not have a sufficient resolving power to resolve the individual molecular 
chains composing polymer crystals, but also because polymer crystals are easily destroyed by electron irradiation. Consequently one had to observe the specimens at low magnifications and/or to investigate them by selected-area electron diffraction, where a small amount of irradiation ought to suffice. Using the replica method or staining method (negative or positive), radiation-resistivity can be improved considerably. However the high resolution images of $2 \sim 3 \AA$ resolution can not be expected with these methods. In 1974, Kobayashi et al. constructed a high resolution EM (JEM-500) with a liquid helium specimen stage, in the Institute for Chemical Research, Kyoto University. This EM has a resolution of $1.4 \AA$ and is to be able to give molecular or atomic images directly, only if the specimens are less radiation-sensitive.

Most of polymer crystals are vulnerable to electron irradiation, and are brought to an amorphous state by a small irradiation dose. Direct imaging of polymer molecules is thus practically difficult with EM, and only two works have been so far done on l-dimensional lattice images of poly (p-phenylene terephthalamide) [PPTA] and poly(p-xylylene) $\left[+\mathrm{CH}_{2}^{16-18}\right.$ PPX ] crystals which are less radiation-sensitive. Both lattice images are cited in Fig.I for PPTA and Fig.2 for PPX. In the case of PPTA, specimens were prepared by fragmentation, where fibers were dispersed by ultrasonic irradiation in water. Niegisch reported both $\alpha$ - and $\beta$-form single crystals of PPX grown from solutions, 
and Bassett \& Keller obtained the dark-field electron micrograph showing the direct image of the $18 \AA$ (10.0) lattice spacing of such a PPX $\beta$-form single crystal. However, it has never been realized to resolve the locations of individual polymer-chains composing polymer single crystals.

We quantitatively studied the damage of PPX crystals under the influence of electron irradiation and found that PPX crystals are rather strong against electron irradiation. ( Their "total end point dose" is about 0.4 Coulombs $/ \mathrm{cm}^{2}$ for $500 \mathrm{KV}$ electrons and about 10 times as large as that of polyethylene crystals. See Chapter 3. ) Thus we have attempted to resolve individual chains of $\mathrm{PPX}$ with JEM-500, and succeeded in direct imaging of the molecular chains of PPX ( see Chapter 3 ).

The crystal structure of the PPX $\alpha$-form has already been analyzed. As regards the PPX $\beta$-form crystal, it was proposed that it belongs to the hexagonal system ( $a=20.52 \AA$, $c$ (fiber axis) $=6.58 \AA$ ), and the molecules are all paralell to the c-axis which is perpendicular to the single crystal end-surface. The detailed crystal structure is not yet known.

We have succeeded in taking high resolution electron micrographs of both PPX $\alpha$-and $\beta$-form single crystals, where we are able to resolve the individual chains composing the $\beta$-form single crystal (projection on the ab-plane). A similarity between the electron diffraction pattern and the optical diffractogram of 


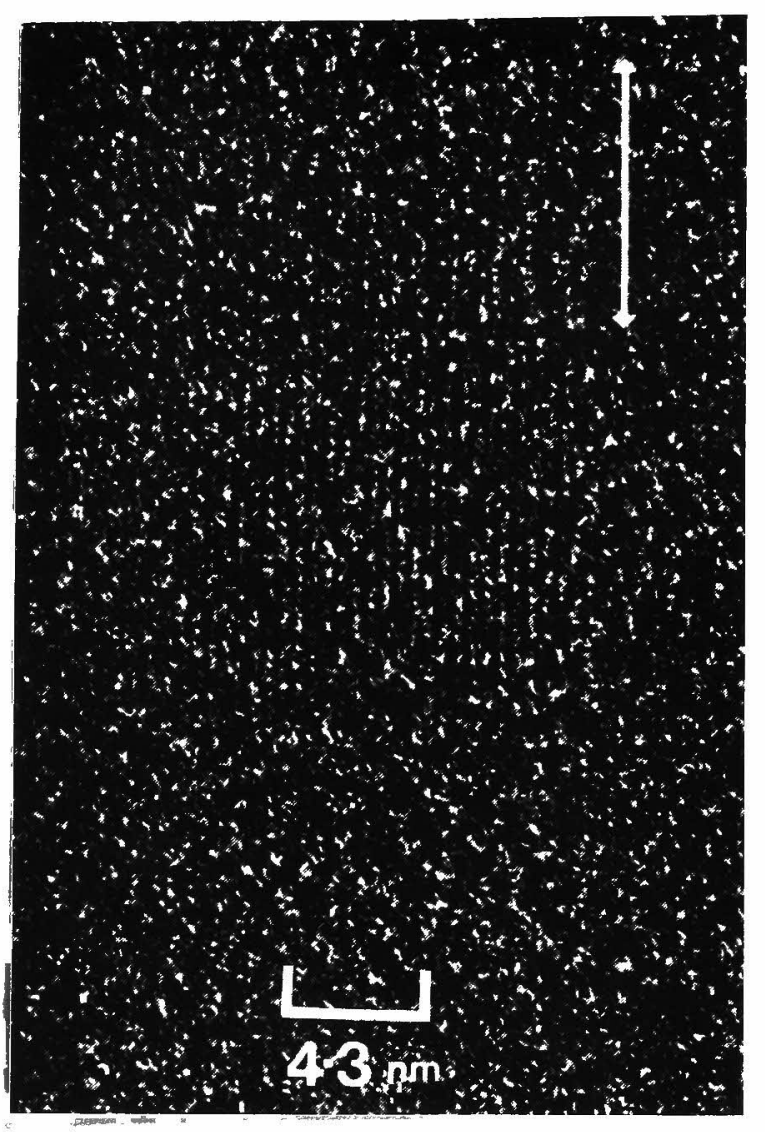

Fig.l Electron micrograph showing $4.33 \AA$ lattice fringes in PPTA (Kevlar 49).

$$
\text { (Dobb et al.) }{ }^{16}
$$

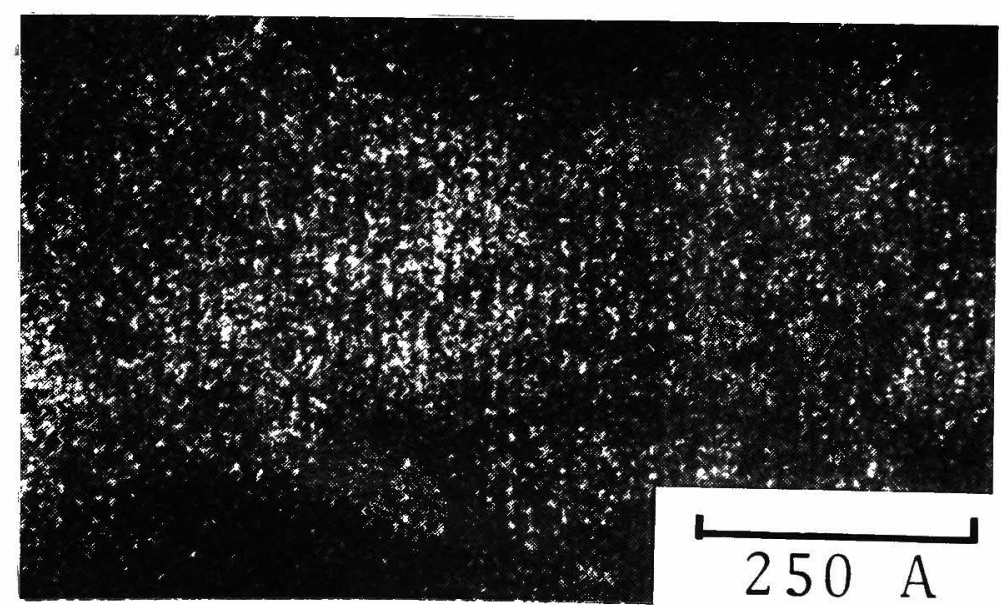

Fig.2 Dark-field micrograph of a PPX $\beta$-form single crystal showing direct image of the $18 \AA$ (10.0) lattice spacing. 19

(Bassett \& Keller) 
the obtained micrograph suggests that the micrograph reflects the crystal structure of the $B$-form with sufficiency. However, because of the low signal-to-noise ratio $(\mathrm{S} / \mathrm{N})$ due to the photographic graininess, the details expected from the diffractogram were not clear. Therefore optical filtering was performed and the periodic structure was accentuated ( see Chapter 4 ).

Ever since Bragg reported the optical analogy of $\mathrm{x}$-ray diffraction patterns in 1939, the optical transform method has been much developed in the field of structure analysis using $x$-ray or electron diffraction ${ }^{23-24}$ on the basis of this principle, Klug et ${ }^{25}$ established the so-called optical filtering method for image processing of electron micrographs, after the method already used in the field of information science. On the other hand, with the advent of the space age, digital image processing for pictures which are transmitted over great distances from far ranging space probes has been rapidly advanced. Such image processing techniques have become indispensable for the high resolving electron microscopy, and image processing systems (both optical and digital) were constructed as a part of our study ( see Chapter 4 ).

A high resolution electron micrograph of a PPX $\beta$-form single crystal was image-processed with the optical filtering system mentioned above. This processed image showed mutual positions of chains in a unit cell in the ab-plane projection. It is concluded from its density and lattice dimensions that a unit cell (hexagonal 
he or trigonal) of the PPX $\beta$-form crystal contains 16 identical 20

luss chain-segments (the segmental length is the fiber period). In

thic polymer crystals with hexagonal or trigonal symmetry, each chain

not should have 3- or 6-fold axis or screw axis ( pseudo-cylindrical

dif symmetry is also permitted, , or otherwise a unit cell must contain an integral multiple of 3 identical chain-segments. The PPX $B$-form ay crystal which does not satisfy this prerequisite is an unusual een example, and the crystal structure has not been analyzed. The high resolution micrograph of the $\beta$-form single crystal indicates the arrangement of molecules in the projection on the ab-plane and gives the starting point of crystal structure analysis of the crystal using $\mathrm{x}$-ray diffraction ( see Chapter 5 ).

While the crystal structure (average periodical structure) can be analyzed by $x$-ray diffraction without causing irradiation problems, non-periodic/irregular structures such as crystal defects ideal structure. All the diffraction methods including $\mathrm{x}$-ray diffraction have such a weak-point. On the other hand, the high resolution $\mathrm{EM}$ which has been developed to have the $2 \sim 3 \AA$ resolution radiation-resisting specimens. However, the defects in polymer crystals have been observed only through moiré fringes and/or 
dislocation networks. The interpretation of them is rather difficult.

We have succeeded in obtaining the molecular images of the $\beta$-form including lattice defects (edge dislocation), and the direct observation of the defects in polymer crystals has become possible for the first time. In our micrograph of the $\beta$-form, the arrangement of molecules in the vicinity of the dislocation core is not distinct, but the appearance of the dislocation is similar to the bubble model of the edge dislocation ( see Chapter 6). A higher resolution image of the dislocation will be able to reveal the manner of the molecular folding as well as the dislocation structure in the near future. 
References

1) A.P.Yundt, TAPPI, 34, 89(1951).

2) W.Schlesinger \& H.M.Leeper, J.Polymer Sci., 11, 203(1953).

3) A.Keller \& J.R.S.Waring, J.Polymer Sci., 17, 447 (1955).

4) R.Jaccodine, Nature, 176, 306 (1955).

5) P.H.Till, J.Polymer Sci., 24, 301 (1957).

6) A.Keller, Phil.Mag., 2, 1171 (1957).

7) E.W.Fischer, Z.Naturforsch., 12a, 753(1957).

8) K.H.Storks, J.Amer.Chem.Soc., 60, 1753(1938).

9) K.H.Storks, Bell Laboratories Record, 21, 390 (1943).

10) K.Herrmann, 0.Gerngross \& W.Abitz, Z.Phys.Chem, B-10, $371(1930)$.

11) E.W.Fischer, Kolloid-Z., 159, 108 (1958).

12) K.Kobayashi, Y.Nishijima, S.Goto \& M.Kurokawa, Proc.4-th Int.Conf.EM, Berlin 1958, Springer-Verlag, 728 (1960).

13) P.H.Geil, "Polymer Single Crystals", Interscience Pub. (1963).

14) B.Wunderlich, "Macromolecular Physics", vol.1, Academic-Press, (1973) .

15) K.Kobayashi, E.Suito, N.Uyeda, M.Watanabe, T.Yanaka, T.Etoh, H.Watanabe \& M.Moriguchi, Proc.8-th Int.Congr.EM, Canberra, vol.1, $30(1974)$

16) M.G.Dobb, A.M.Hindeleh, D.J.Johnson \& B.P.Saville, Nature, 253, $189(1975)$. 
17) S.C.Bennett, M.G.Dobb, D.J.Johnson, R.Murray \& B.P.Saville, Proc.EMAG-75, Bristol, $329(1976)$.

18) M.G.Dobb, D.J.Johnson \& B.P.Saville, J.Polymer Sci.:Polymer Symp., 58, 237 (1977).

19) G.A.Bassett \& A.Keller; cited by A.Keller, Kolloid-Z., 231, 386 (1969) .

20) W.D.Niegisch, J.Appl.Phys., 37, 4041(1966).

21) R.Iwamoto \& B.Wunderlich, J.Polymer Sci.-phys., 11, 2403(1973).

22) W.L.Bragg, Nature, 143, 678(1939).

23) C.A.Taylor \& H.Lipson, "Optical Transforms", G.Bell \& Sons, (1964).

24) H.Lipson ed., "Optical Transforms", Academic-Press, (1972).

25) A.Klug \& D.J.DeRosier, Nature, 212, 29 (1966).

26) E.L.O'Neill, I.R.E.Trans.Int.Theory, IT-2, 56 (1956).

27) R.Nathan, in "Advances in Optical and Electron Microscopy", R.Barer \& V.E.Cosslett Ed., vol.4, Academic-Press, 85 (1971).

28) M.Tsuji, S.Isoda, M.O'hara, K.Katayama \& K.Kobayashi, Bull.Inst.Chem.Res., Kyoto Univ., 55, 237 (1977).

29) for example, see "Direct Imaging of Atoms in Crystals and Molecules", Ed. by L.Kihlborg, (Proc. 47-th Nobel Symposium, Lidingö), Royal Swedish Academy of Sciences, (1979).

30) V.F.Holland, J.Appl.Phys., 35, 3235 (1964).

31) P.H.Lindenmeyer, J.Polymer Sci., C-15, 109 (1966).

32) W.L.Bragg \& J.F.Nye, Proc.Roy.Soc., A-190 ,474 (1947). 
Chapter 1 : Some aspects of high resolution electron microscopy

Provided that a light microscope has an ideal objective lens without any kinds of aberrations, the resolution limit of a light microscope with illumination parallel to the optic axis is given according to Abbe's theory by

$$
\mathrm{d}_{\mathrm{D}}=\mathrm{k} \lambda / \sin \alpha_{\max },
$$

where $\mathrm{d}_{\mathrm{D}}$ is the minimum distance between two light-absorbing particles that can be recognized as separate in the image, $\lambda$ is the wavelength of light, $\alpha_{\max }$ is the maximum aperture angle of the objective lens, and $\mathrm{K}$ is the constant; $\mathrm{K}=0.61$ for incoherent illumination, or $\mathrm{K}=0.77$ for coherent illumination. Since $\alpha_{\max }<\pi / 2$, then $d_{D}>k \lambda$. Therefore the resolution of a microscope never exceeds about half a wavelength of the wave which we may use. In electron optics the relation between aperture angle and 
resolving power is more complicated. Electron lenses used as an objective lens cannot be corrected spherically, though they can be almost stigmated by stigmator. Electron waves passing through the outer zones of the lens miss the Gaussian image point. The resolution limit only due to spherical aberration is given by,

$$
d_{s}=c_{s} \alpha^{3}
$$

where $C_{S}$ is a spherical aberration coefficient and $\alpha$ is the aperture angle. The intensity distribution on the image plane corresponding to a point object in the object plane may be considered as a Gaussian distribution whose half-breadth corresponds to the "resolution limit". Therefore the resolving power $d$ of an electron microscope (EM) may be approximately estimated from both Eqs.(1-1) and (1-2), using the following equation:

$$
d^{2}=d^{2}+d_{s}^{2} \text {. }
$$

Since $\alpha$ is considered sufficiently small, the optimum aperture angle $\alpha_{\text {opt }}$ and the resolution limit $\mathrm{d}_{\text {opt }}$ are given as follows (for $\mathrm{K}=0.61$ ):

$$
\begin{aligned}
& \alpha_{\text {opt }}=0.77\left(\lambda / \mathrm{C}_{\mathrm{s}}\right)^{1 / 4}, \\
& \mathrm{~d}_{\text {opt }}=0.91\left(\lambda^{3} \mathrm{C}_{\mathrm{s}}\right)^{1 / 4} .
\end{aligned}
$$

For $100 \mathrm{KV}$ electrons $(\lambda=0.037 \AA)$ and $\mathrm{C}_{\mathrm{s}}=1.4 \mathrm{~mm}$,

$$
\mathrm{d}_{\text {opt }} \cong 4.7(\AA) \text {. }
$$

This simple estimation is applicable to the amplitude contrast due to so-called "absorption" effect of a specimen. In high resolution electron microscopy, however, very thin specimens which 
can be considered as phase objects, are observed using high accelerating voltage. In this case, we must estimate the resolution limit from the standpoint of the phase contrast based on the Scherzer's treatment. The image contrast of phase objects ought to be very weak on Gaussian image plane. Therefore, as Scherzer described, a small amount of defocusing is beneficial to the contrast, and the optimum defocus is related to the spherical aberration. The true resolving power is inherently limited by the wavelength, maximum aperture angle, illuminating angle, and energy spread as shown later.

In this chapter, we will describe the imaging theory in high resolution electron microscopy and then some problems in taking high resolution electron micrographs.

1-1 Scattering of electrons through the objects

If a periodic object (thickness: R) consists of stacked thin-slices each of which is expressed by an 1-dimensional transmission function $\cos (2 \pi x / d)$ with the period of $d$, the phase difference between the wave $\psi_{1}$ which was scattered by the first slice and propagated the distance $R$ and the wave $\psi_{2}$ scattered for the first time by the last slice is given by $\pi R \lambda / d^{2}{ }^{42}$ This phase difference must be within $\pi / 2$ according to Cowley's criterion to 
avoid negative interference of the waves. Then in order to resolve a distance $d_{\min }$, the specimen thickness must not exceed, approximately

$$
\mathrm{R}_{\max }=\mathrm{d}_{\min }^{2} / 2 \lambda
$$

This equation is derived considering the effect of the Fresnel diffraction. For $\lambda=0.0142 \AA$ (500KV electrons), Eq.(1-6) gives $\mathrm{d}_{\min }=2 \AA$ for $\mathrm{R}_{\max }=282 \AA$, and $\mathrm{d}_{\min }=4 \AA$ for $\mathrm{R}_{\max }=1127 \AA$. When $\mathrm{a}$ specimen is sufficiently thin in relation to the EM resolution, then the specimen may be considered as a 2-dimensional object according to the criterion mentioned above. Its effects on the electron beam will be represented by changes of phase and amplitude which may be considered to take place on a single plane. Thus the scattering from this object can be treated kinematically. The wave function of the incident wave, assumed to be coherent and of amplitude unity, will be multiplied by the 2-dimensional transmission function of this planar object, which may be written:

$$
q\left(x_{0}\right)=\exp \left\{-i \sigma \phi\left(x_{0}\right)-\mu\left(x_{0}\right)\right\} \quad \text {. }
$$

Here $\sigma$ is the interaction constant $(\sigma=\pi / \lambda \mathrm{E}$ and $\mathrm{E}$ is the accelerating voltage $). \phi\left(x_{0}\right)$ and $\mu\left(x_{0}\right)$ are the projections in the beam direction of the 3-dimensional potential distribution $\phi\left(x_{0}, z\right)$ and effective absorption function $\mu\left(x_{0}, z\right)$ of the specimen, respectively, so that

and

$$
\phi\left(x_{0}\right)=\int \phi\left(x_{0}, z\right) d z \text {, }
$$

$$
\mu\left(x_{0}\right)=\int \mu\left(x_{0}, z\right) d z
$$


For a particularly thin object or sufficiently high accelerating voltage, $\mu\left(x_{0}\right)$ can be neglected and the specimen can be treated as a pure phase object. If the specimen is composed of light atoms, $\phi\left(x_{0}\right)$ is sufficiently small, and only the first order term is significant in the expansion of the exponential in Eq.(1-7):

$$
q\left(x_{0}\right) \cong 1-i \sigma \phi\left(x_{0}\right)
$$

In this case, the object is called a "weak phase object". The first term of Eq. (1-8) represents the unscattered incident wave and the second one the scattered wave. The Fourier transform $Q$, of this function $q$, is given by:

$$
\begin{aligned}
Q(u) & =\int q\left(x_{0}\right) \exp \left\{2 \pi i u x_{0}\right\} d x_{0} \\
& =\delta(u)-i \sigma \Phi(u),
\end{aligned}
$$

where $\Phi(u)=\int \phi\left(x_{0}\right) \exp \left\{2 \pi i u x_{0}\right\} \quad d x_{0}$, or for periodic objects,

$$
\Phi(u) \equiv(\lambda / \sigma) F(h, k)=(\lambda / \sigma) \sum_{j} f_{j}(h, k) \exp \left\{2 \pi i\left(h x_{0}+k y_{0}\right)\right\},
$$

$\left(\mathrm{h}, \mathrm{k}\right.$ : Miller indices, $f_{j}(h, k)$ : atomic scattering factor of $j$-th atom for electrons, $x_{0}, y_{0}$ : fractional coordinates ).

1-2 Image formation in high resolution electron microscopy

The objective lens of EM transfers the electron waves, which have just transmitted through an object, to form its diffraction pattern (viz. spatial frequency distribution spectrum of the object) 
at the back focal plane and its inverted real image at the Gaussian image plane, as is shown in the textbooks of ordinary physical optics. The total process can be interpreted in terms of two successive Fourier transforms. The amplitude transmittance of an object corresponds to the wave transmitted through the object, as described in the previous section. With the coordinates assigned as in Fig.1-1, the amplitude $E\left(X_{f}\right)$ ( at the back focal plane of the objective lens, of the electron wave transmitted through an object will be given by the following equation

$$
\begin{gathered}
E\left(x_{f}\right)=(i / \lambda f) \exp \left\{-i k\left(f+d_{1}-\Delta f\right)-i k\left(x_{f}^{2} / 2 f\right) \cdot\left(1-d_{1} / f\right)\right\} \cdot Q\left(x_{f} / \lambda f\right) \\
\quad x \exp \left\{-i k \Delta f x_{f}^{2} / 2 f^{2}\right\}
\end{gathered}
$$

where $\Delta f$ is the amount of defocusing and positive for under focusing, and $k=2 \pi / \lambda$. The last factor in Eq. (1-10) is the wave aberration due to the defocusing $\Delta f\left(\right.$ distances $d_{2}$ and $\left(d_{1}-\Delta f\right)$ in Fig.1-1 are fixed in EM, where $d_{1}$ and $d_{2}$ are a pair of conjugate distances with respect to the objective lens). The intensity of the diffracted wave, namely the diffraction intensity at the back focal plane, is given by $\left|E\left(x_{f}\right)\right|^{2}$. Then from Eq. (1-10),

$$
\left|E\left(x_{f}\right)\right|^{2}=\left(1 / \lambda^{2} f^{2}\right) \cdot\left|Q\left(x_{f} / \lambda f\right)\right|^{2} \text {. }
$$

Including both spherical and defocusing aberration, the aberration function $\chi$ is defined as follows:

$$
x\left(x_{f} / \lambda f\right) \equiv \pi \lambda \cdot \Delta f \cdot\left(x_{f}^{2} / \lambda^{2} f^{2}\right)-(\pi / 2) c_{s} \lambda^{3} \cdot\left(x_{f}^{2} / \lambda^{2} f^{2}\right)^{2},
$$

where $C_{S}$ is the spherical aberration coefficient.

Thus Eq. (1-10) can be modified as follows: 


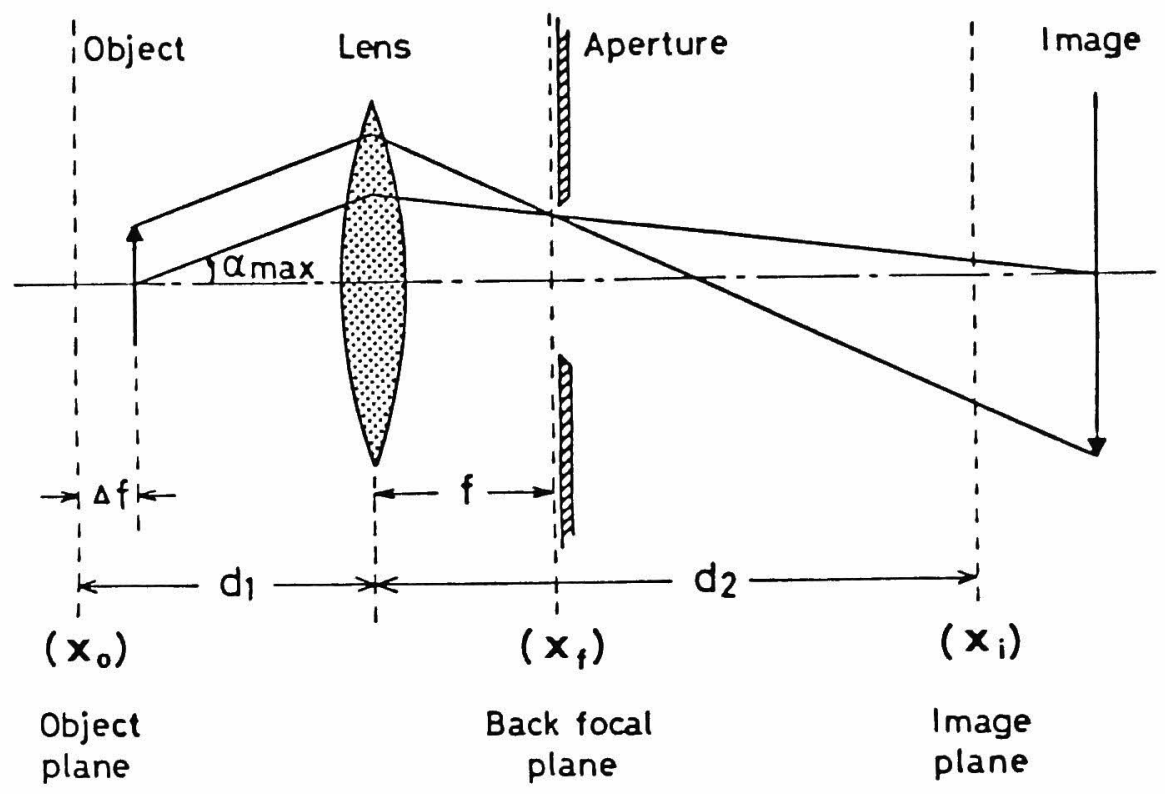

Fig.1-1 Image formation by an objective lens in EM, $\alpha_{\max }:$ maximum aperture-angle, $f$ : focal length of the lens,

$\Delta \mathrm{f}$ : amount of defocusing (positive for under-focus). 


$$
\begin{gathered}
E\left(x_{f}\right)=(i / \lambda f) \exp \left\{-i k\left(f+d_{1}-\Delta f\right)-i k\left(x_{f}^{2} / 2 f\right) \cdot\left(1-d_{1} / f\right)\right\} \cdot Q\left(x_{f} / \lambda f\right) \\
\quad \times \exp \left\{-i x\left(x_{f} / \lambda f\right)\right\} .
\end{gathered}
$$

Then the amplitude $\Psi\left(x_{i}\right)$ at the image plane is given by the diffraction integral, namely the Fresnel diffraction, of $E\left(x_{f}\right) \cdot A\left(x_{f} / \lambda f\right)$, where $A$ is the aperture function. Therefore

$$
\begin{aligned}
\Psi\left(x_{i}\right) & =(-I / M) \exp \left\{-i k\left(f+d_{1}-\Delta f\right)-i k x_{f}^{2} / 2\left(d_{2}-f\right)\right\} \\
& \times \int Q(u) \exp \{-i x(u)\} A(u) \exp \left\{2 \pi i x_{i} u / M\right\} d u, \quad(1-14)
\end{aligned}
$$

where $M$ is the magnification ( $f f . M=d_{2} / d_{1}$ ) and $u\left(=x_{f} / \lambda f\right.$ ) is the spatial frequency. The intensity $I\left(x_{i}\right)$ of the image is given by

$$
\begin{aligned}
I\left(x_{i}\right) & =\left|\Psi\left(x_{i}\right)\right|^{2} \\
& =\left|\psi\left(x_{i}\right)\right|^{2} / M^{2},
\end{aligned}
$$

where

$$
\psi\left(x_{i}\right) \equiv \int Q(u) \exp \{-i x(u)\} A(u) \exp \left\{2 \pi i x_{i} u / M\right\} d u \quad .
$$

If we use a circular objective aperture and an aberration function with cylindrical symmetry, both functions for $u=|u|$ are as follows :

$$
A(u)= \begin{cases}1 ; & \text { for } u \leq u_{\max } \\ 0 ; & \text { for } u_{>} u_{\max },\end{cases}
$$

and

$$
X(u)=\pi \lambda \cdot \Lambda f u^{2}-(\pi / 2) c_{n} \lambda^{3} u^{4},
$$

where $u_{\text {max }} \cong \alpha_{\text {max }} / \lambda ; \alpha_{\max }$ is the maximum aperture angle of the objective lens. Noting that the potential function $\phi(x)$ is real, we obtain from Eqs. (1-9) and $(1-15)$,

$$
I\left(x_{i}\right) \cong\left(1 / M^{2}\right)\left[1-2 \int \sigma \Phi(u) \sin x(u) A(u) \exp \left\{2 \pi i x_{i} u / M\right\} d u\right],(1-18)
$$


where $\sin X(u)$ is called the "phase contrast" transfer function. This equation indicates that the image intensity $I\left(x_{i}\right)$ is greatly affected by defocusing through $\sin x(u)$ in the case of phase contrast. If $\sin \chi(u)= \pm l$ and $A(u)=l$ for all $u$, then

$$
I\left(x_{i}\right)=\left(I / M^{2}\right)\left[I \mp 2 \sigma \phi\left(-x_{i} / M\right)\right] \text {, }
$$

and we thus obtain the magnified and inverted real image which is to reflect the potential distribution $\phi$, viz. the true structure of the object. Under actual circumstances, $\sin \chi(u)$ oscillates and here we define $u_{1}$ as the smallest value of $u$ (except $u=0$ ) given by $\sin X(u)=0$. For high resolution electron microscopy, we must operate EM at the "optimum defocus" which gives $|\sin X(u)|$ its maximum value 1 over a range of $u$ as wide as possible. Figure $1-2$ shows several curves of $\sin \chi(u)$ for $\mathrm{C}_{\mathrm{s}}=1.06 \mathrm{~mm}$ and $\lambda=0.0142 \AA$ (500KV electrons). The aperture size $u_{\max }$ should be selected under the optimum-defocus condition so as to cut-off all the scattering waves in the higher spatial frequency range where $\sin \chi(u)$ oscillates violently. It may be the best to select $u_{1}$ at the optimum defocus as $u_{\max }$ - Then $1 / u_{\max }$ corresponds to the resolving power. Scherzer reported the resolution limit with his optimum-defocus criterion:

$$
d=0.60\left(C_{s} \lambda^{3}\right)^{1 / 4},
$$

for axial illumination. Though various criterions have been proposed to determine the optimum-defocus condition, the resulting resolution limits little differ. For JEM-500 $\left(C_{S}=1.06 \mathrm{~mm}\right.$, 
$\lambda=0.0(42 \AA)$, in the Institute for Chemical Research, Kyoto University, the resolution limit is estimated at $1.41 \AA$ from Eq. $(1-20)$.

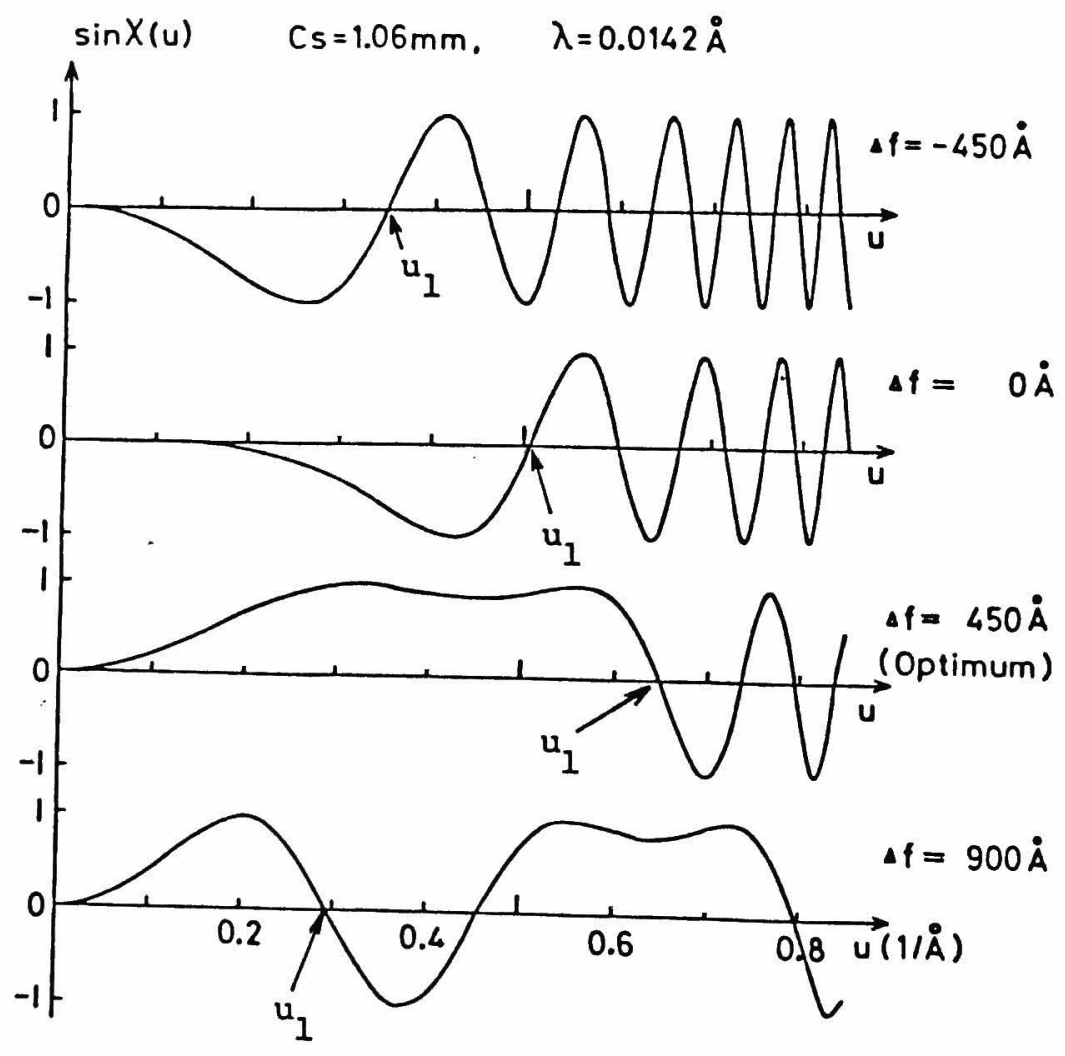
Fig.1-2 Phase contrast transfer function $\sin \chi(u)$ of JEM-500
$\left(C_{s}=1.06 \mathrm{~mm} ; \lambda=0.0142 \AA\right)$. 
1-3 Some problems in taking high resolution electron micrographs

In the previous section, we described the phase contrast image formation theory and the resolution limit in high resolution electron microscopy. In this section, some problems in obtaining high resolution micrographs of PPX single crystals with JEM-500 will be discussed.

There are many factors which restrict the resolution limit of EM. The distance between polymer chains in PPX crystals is about $5 \AA$ as shown in Chapter 2. However, $2 \AA$ resolution will be needed at least to obtain the information on molecular arrangement in the crystal. Principal factors affecting the resolution of individual chains composing a polymer single crystal are summarized as follows:

(1) Specimen

1) Thickness

2) Orientation

3) Radiation damage

4) Specimen drift

(2) EM

5) Spherical aberration of objective lens

6) Energy spread and Illuminating angle

7) Astigmatism of objective lens

8) Recording systems 


\section{1-3-1 Specimen thickness}

Both $\alpha$ - and $\beta$-form PPX single crystals are about $100 \AA$ thick as shown in Chapter 2. From Eq. $(1-6), R_{\max }=282 \AA$ when $d_{\min }=2 \AA$, that is, $R_{\max }$ is larger than the thickness of the specimen. Therefore the resolution higher than $2 \AA$, namely the resolution of individual polymer chains composing PPX single crystals, can be expected from the standpoint of the Fresnel diffraction effect ( see section 1-1).

According to the two-beam dynamical theory, the extinction distance is approximately given by

$$
\xi_{\mathrm{g}}=\pi \mathrm{V}_{\mathrm{c}} \cdot \cos \theta / \lambda \mathrm{F}_{\mathrm{g}} \quad \text {, }
$$

where $V_{c}$ is the unit cell volume; $\theta$ is the Bragg angle; and $F_{g}$ is the structure factor corresponding to the diffracting direction $g$. For example, in the case of the PPX $\beta$-form crystal (see Chapter 5 ), $V_{C} \cong 2400 \AA, F_{40.0} \cong 340 \AA$, and $\cos \theta \cong 1.0$. It follows from Eq. (1-21) that $\xi_{40.0} \cong 1600 \AA$ for $500 \mathrm{KV}$ electrons $(\lambda=0.0142 \AA)$. The thickness of PPX single crystals is much smaller than $\xi_{40.0} / 2$, so that the scattering of electrons through them can be treated kinematically. If the specimen is rather thick, a dynamical scattering effect (multiple scattering, and so on ) will be remarkable. Then EM images do not directly reflect the crystal structure ( namely, the projected potential distribution ). 


\section{1-3-2 Specimen orientation}

As described in Chapter 2, a thin single-layer crystal of the PPX $\alpha$-form gives the selected area diffraction pattern corresponding to $\langle 102\rangle$ incidence of electrons. The molecular axis of this crystal is not perpendicular to the crystal end-surfaces. Therefore we cannot expect the resolution of individual chains in this crystal.

In a PPX $\beta$-form single crystal, all the molecules align perpendicular to the crystal end-surface, so that the individual chains composing this crystal may be resolved.

\section{1-3-3 Radiation damage of the specimen}

The total end point dose for $500 \mathrm{KV}$ electrons is about 0.4 Coulombs $/ \mathrm{cm}^{2}$ for both $\alpha$ - and $\beta$-form PPX crystals ( see Chapter 3). This value corresponds to the exposure to produce one or two micrographs at a magnification $M=10^{5} \mathrm{X}$. Though more micrographs can be taken from one crystal at a lower magnification, the granularity of the recording medium (photographic films) requires a magnification of $10^{5} \mathrm{x}$ to achieve $2 \AA$ resolution.

\section{1-3-4 Specimen drift}

We should avoid the mechanical stage drift after specimen translation as well as the focus drift due to electrical hysteresis of the intermediate lens after changing from a diffraction mode to 
an imaging mode. Photographing is postponed for $3 \sim 5$ minutes, at least, to avoid the above-mentioned drifts.

We should also suppress the specimen drift due to thermal deformation and charge-up of the specimen and/or support-film with applying electrons. A carbon- and gold-coated microgrid (holy film) is used, on which a very thin carbon supporting film is deposited, as described in Chapter 3.

If the energy from the incident beam is uniformly deposited throughout the cylindrically irradiated volume and the front and back of the thin specimen are at the same temperature, then the heat flow is considered purely radial. According to Isaacson, the temperature rise $\Delta T\left({ }^{\circ} \mathrm{C}\right)$ of the support-film in the center of the irradiated volume is given as follows:

$$
\Delta \mathrm{T} \cong 1.2 \times 10^{-14} \mathrm{~N}_{\mathrm{b}} / \beta^{2} \mathrm{~K}\left[\ln \left(r_{\mathrm{G}} / \mathrm{r}_{\mathrm{B}}\right)+1 / 2\right],
$$

where $\mathrm{N}_{\mathrm{b}}$ is the beam current in electrons/sec on the specimen, $\mathrm{K}$ is thermal conductivity in $\mathrm{cal} / \mathrm{cm} / \mathrm{sec} /{ }^{\circ} \mathrm{C}, \beta=\mathrm{v} / \mathrm{c}$ is the ratio of the electron velocity $v$ to the light velocity $c . r_{G}$ denotes the radius of hole openings in a metallic specimen-grid and $r_{B}$ the radius of a cylindrical electron beam which illuminates the specimen grid in the center of a grid hole. For ordinary illumination condition, $r_{G} / r_{B} \cong 1$. We use the following conditions: $\beta=0.8629$ for $500 \mathrm{KV}$ electrons, and $\mathrm{N}_{\mathrm{b}}=3 \times 10^{13}$ electrons/sec for a magnification of $10^{5} \mathrm{X}$ and the grid of $200 \mathrm{mesh} / \mathrm{inch}$. Since the thermal conductivity for gold is $0.708 \mathrm{cal} / \mathrm{cm} / \mathrm{sec} / \mathrm{deg}\left(20^{\circ} \mathrm{C}\right), \Delta \mathrm{T} \cong 0.3\left({ }^{\circ} \mathrm{C}\right)$ for very thin 
gold films. Thus the temperature rise is minimal and we are able to reduce the radiation damage of specimens with the gold-coated microgrid.

We are not willing to use a carbon support-film on the microgrid, because it gives an extra noise on EM images. However, it suppresses the electron charge-up on the specimen and other effects which are shown in Chapter 3.

1-3-5 Spherical aberration of objective lens

The effect of this aberration was described in section 1-2. An electron micrograph should be taken under the optimum-defocus condition in order to obtain an image which reflects the true structure of the object. About $450 \AA$ under-focus is the optimum defocus for JEM-500 according to Cowley's criterion:

$$
\Delta f_{\text {opt }}=\left\{(4 / 3) c_{s} \lambda\right\}^{1 / 2} \text {. }
$$

1-3-6 Energy spread and Illuminating angle

The illuminating angle and the parameter for energy spread are to modify the phase contrast transfer function $\sin X(u), i . e .$, this function should be multiplied by two envelope functions $S(u)$ and $E(u)$ which are expressed in terms of the illuminating angle and the energy spread as

$$
s(u)=\exp \left[-\left(\pi \beta_{i} / \lambda\right)\left(c_{s} \lambda^{3} u^{2}-\Delta f \lambda\right)\right],
$$

and 


$$
\begin{aligned}
E(u) & =\exp \left[-\left(\pi \lambda u^{2} / 2\right)\left(C_{C} \cdot \delta E / 2 E \sqrt{\ln 2}\right)\right] \\
& =\exp \left[-\left(\pi \lambda \Delta \cdot u^{2} / 2\right)\right]
\end{aligned}
$$

where $\beta_{i}$ is the illuminating angle; $\Delta f$ the defocus; $C_{C}$ the chromatic aberration constant; $\delta \mathrm{E} / \mathrm{E}$ the fluctuation of accelerating voltage; and $\Delta$ the parameter for energy spread. For JEM-500,

$\Delta \cong 74 \AA$ and $\beta_{i} \cong 2 \times 10^{-4} \mathrm{rad}$ under the best condition. Since two envelope functions damp $|\sin X(u)|$ to considerably small value when $u>u_{\max }\left(u_{\max }\right.$ is given from Scherzer's limit (Eq.(1-20)), practically we need not use the objective aperture.

\section{1-3-7 Astigmatism of objective lens}

In order to obtain high resolution electron micrographs with the resolution better than $2 \AA$, the astigmatism of the objective lens must be compensated within about $100 \AA$, because of the short depth of focus of JEM-500. Owing to a number of causes the resolution of the image seen on the viewing screen of the high voltage EM is too poor to permit compensation with such an accuracy. The method for accurate astigmatism-correction in the present study is as follows:

Astigmatism is most easily recognized from the optical diffraction pattern of a high resolution electron micrograph of a 15

thin amorphous film. As a perfectly stigmated micrograph would give a diffraction pattern consisting of concentric rings due to $\sin ^{2} x(u)^{17}$, departure from circularity indicates the presence of astigmatism. The usual pattern of contrast transfer zones and gaps 
is shown in Fig.1-3(a), which is an optical diffractogram from the micrograph of a thin carbon film before the astigmatism correction. The gaps appear at spatial frequencies u's which satisfy

$$
X(u)=n \pi \quad(n ; \text { integer }) .
$$

Here the aberration function $\chi(u)$ is given from Eq. (1-17) as

$$
\chi(u)=\pi \lambda \cdot \Delta E \cdot u^{2}-(\pi / 2) c_{s} \lambda^{3} u^{4} .
$$

In a high voltage $\mathrm{EM}$ with small $\mathrm{C}_{S}$, only the second term in Eq.(1-17) is significant for spatial frequencies higher than $0.3 \AA^{-1}$. When the first term dominates, the position of the transfer gaps are given by

$$
u_{n}=\sqrt{n /|\Delta f| \lambda}
$$

The defocus is then estimated from the following equation:

$$
|\Delta \mathrm{E}|=\mathrm{L}^{2} \mathrm{n} \Lambda^{2} / \mathrm{M}^{2} \mathrm{r}_{\mathrm{n}}^{2} \lambda
$$

where $M, \Lambda, r_{n}$ and $L$ are the electron optical magnification, the He-Ne gas laser wavelength $(\Lambda=6328 \AA)$, the radius of the $n$-th gap, and the camera length of the apparatus for optical transformation, respectively. The defocus is measured for each principal astigmatism direction to determine the astigmatism $\delta \mathrm{f}$ which is equal to the difference between two principal values. The astigmatism is given in terms of observable $r_{n s}, r_{n l}$, and $\theta$ ( see Fig.1-3(a), as:

$$
\delta \mathbf{f}=\left(\mathrm{n} \Lambda^{2} \mathrm{~L}^{2} / \mathrm{M}^{2} \lambda\right) \cdot\left(1 / \mathrm{x}_{\mathrm{ns}}^{2}-1 / \mathrm{x}_{\mathrm{nl}}^{2}\right) \text {. }
$$

When the $x$ - and $y$-axis of the stigmator make an angle of $\pi / 4$, the correction values of the stigmator currents are: 


$$
\begin{aligned}
& \Delta I_{x}=C \cdot \delta f \sin (|\theta-\alpha|-\pi / 4), \\
& \Delta I_{y}=C \cdot \delta f \sin (|\theta-\alpha+\pi / 4|-\pi / 4)
\end{aligned}
$$

Here $\alpha$ and $C$ are calibration constants to be adjusted for individual EM's. In our case, $C=1 / 137$, and $\alpha=101^{\bullet}$ for $M=10^{5} \mathrm{X}$. For other magnifications, the $\alpha$-value is as given in Table 1-l. Figure 1-3(b) shows the result of astigmatism correction according to the procedure mentioned above. The practical procedure is shown in Chapter 3. If the image intensity is recorded with other methods than photographic one, the real-time stigmating can be done with a similar scheme mentioned in this section.

\section{1-3-8 Recording systems}

In high resolution transmission electron microscopy, the best medium for image recording now available is the photographic emulsion. Although various recording devices have been proposed for radiation-sensitive specimens, the resolution of these devices is generally inferior to that of photographic emulsion.

Among various photographic films and plates analysed, KODAK electron image films and FUJI electron-microscopic films are available in Japan. Figure 1-4 shows the characteristic curves of both films for $500 \mathrm{KV}$ electrons where development condition is shown in the figure caption. The characteristic curve of KODAK electron image film SO-163 shows rather good linearlity. Though KODAK AA-5 $\mathrm{X}$-ray films are several times as sensitive as so-163 image films as 


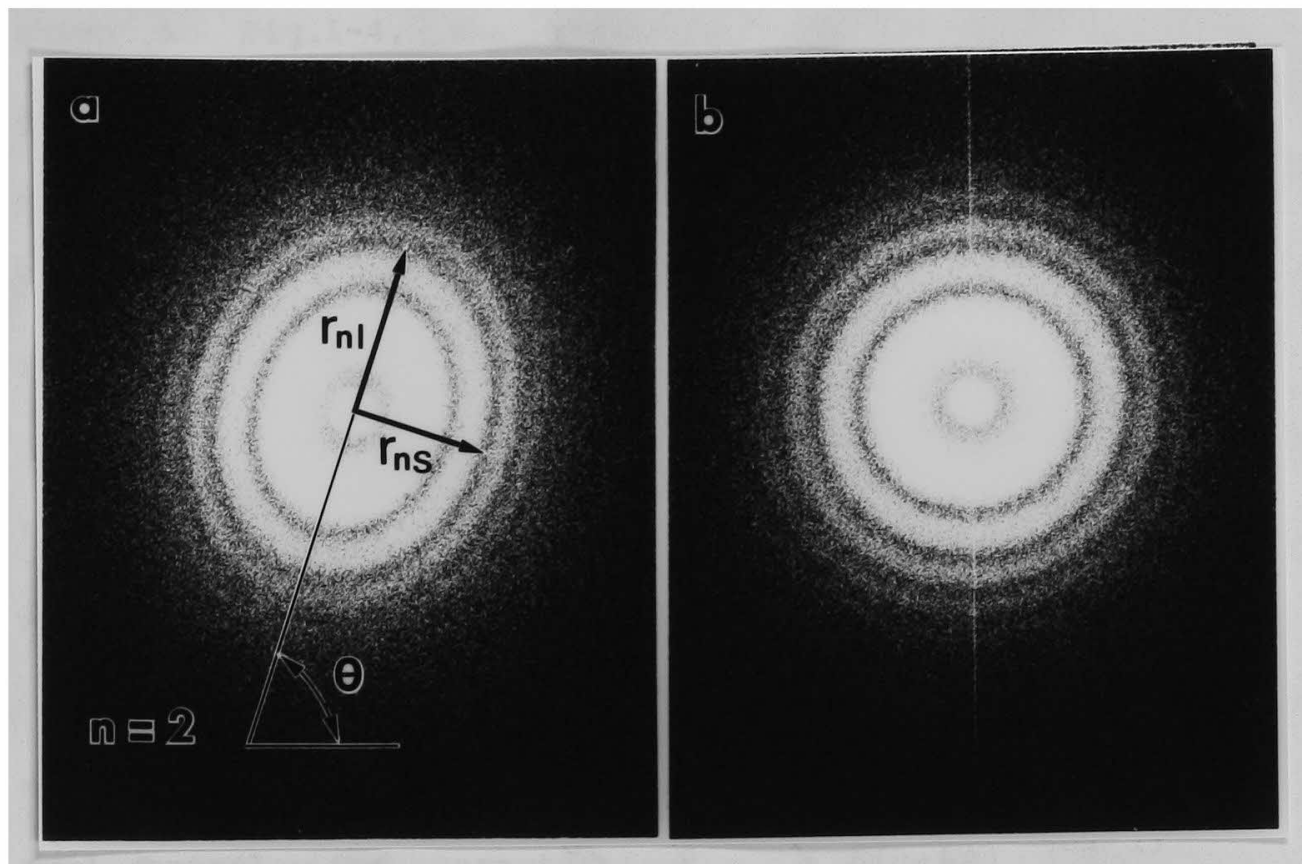

Fig.1-3 Optical diffractograms of images of a thin carbon film: (a) before and (b) after astigmatism correction.

Table 1-1

Calibration constant $\alpha$ of JEM-500 for accurate correction of objective lens astigmatism

\begin{tabular}{lcc}
$\begin{array}{c}\text { Magnification } \\
\text { mode }\end{array}$ & $\begin{array}{c}\text { Magnification } \\
(\times 10,000)\end{array}$ & $\begin{array}{c}\alpha \\
(\text { degree })\end{array}$ \\
\hline \multirow{2}{*}{ M3 } & 5 & 111 \\
& 10 & 101 \\
15 & 91 \\
20 & 82 \\
& 30 & 61 \\
& 40 & 45 \\
\hline \multirow{2}{*}{ M4 } & 12 & 121 \\
& 25 & 110 \\
& 48 & 103 \\
& 54 & 90 \\
& 80 & 70
\end{tabular}




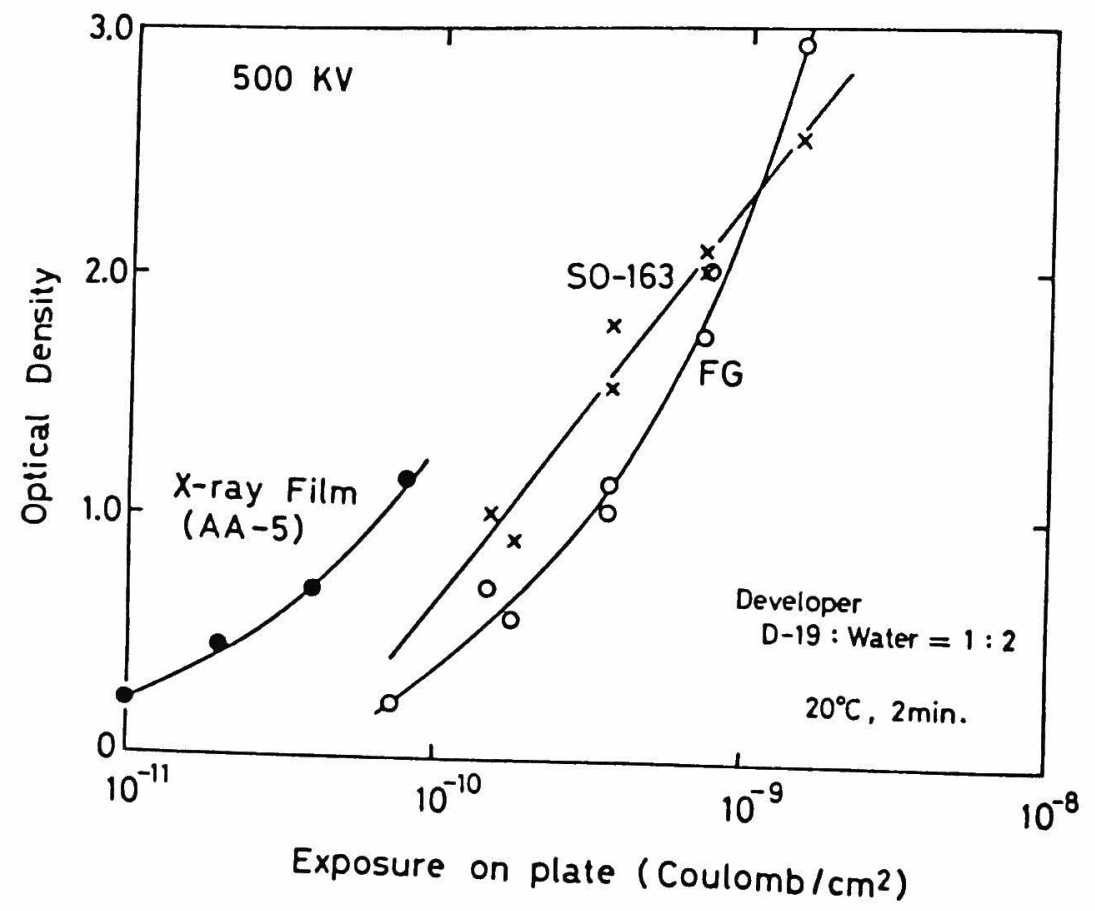

Fig.1-4 Characteristic curves of several photographic filmes for $500 \mathrm{KV}$ electrons

(developed at $20^{\circ} \mathrm{C}$ for 2 minutes with KODAK D-19 diluted $1: 2$ ).

$x$ : KODAK electron image film So-163,

- : FUJI electron-microscopic film FG,

- : KODAK X-ray film AA-5. 
shown in Fig.1-4, the resolution of these x-ray films is approximately $1 / 10$ of image films. The image quality of so-163 is more satisfactory than that of others. Therefore we employed the KODAK SO-163 electron image films to record high resolution images for the subsequent works. The films were developed at $20^{\circ} \mathrm{C}$ for 3 minutes with KODAK Developer D-19 diluted 1:1 ( see Chapter 3). Figure 1-5 shows the characteristic curve of so-163 under such a developing condition. The KODAK electron image films 4463 were also used. Here the film property of 4463 is very similar to so-163, except for the back of 4463 coated with a thin gelatin layer for curl control. The characteristic curve of this film is also plotted in Fig.1-5 under the same developing condition.

High resolution electron micrographs taken in this work had the averaged optical density of about 0.2. The optical density $D$ may be approximately proportional to the electron exposure $\mathrm{E}$ up to $\mathrm{D} \cong 1.0$. This density range is desirable for the improvement of image quality by image processing (Chapter 4 ). 


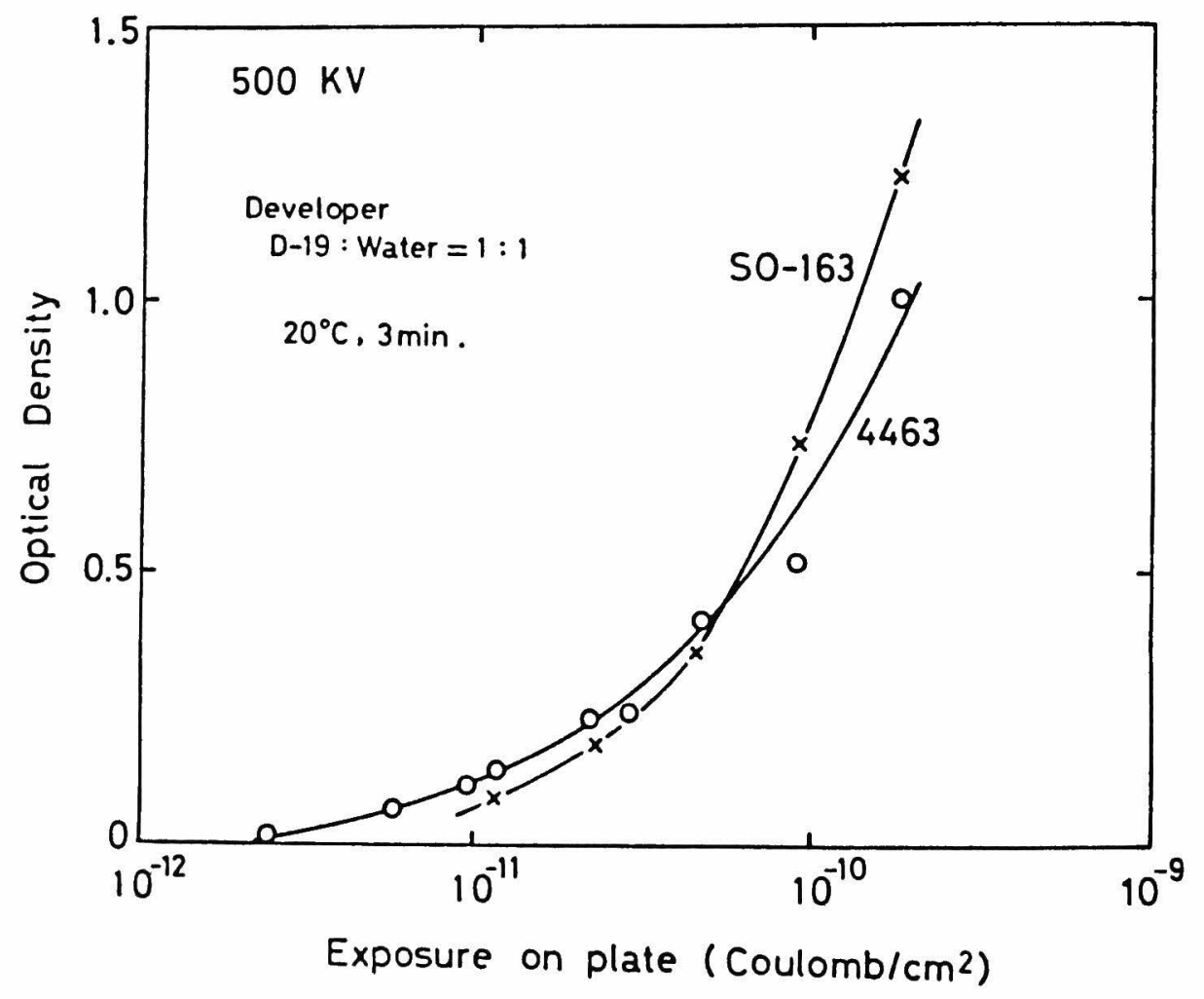

Fig.1-5 Characteristic curves of KODAK electron image films for $500 \mathrm{KV}$ electrons

(developed at $20^{\circ} \mathrm{C}$ for 3 minutes with KODAK D-19 diluted $1: 1$ ).

$\mathrm{x}:$ so-163, $\quad$ : $: 4463$. 
References

1) M.Born \& E.Wolf, "Principles of Optics", 5-th ed., Pergamon-Press, Chapter 8, (1975).

2) S.G.Lipson \& H.Lipson, "Optical Physics", Cambridge Univ.Press, Chapter 9, (1969).

3) V.E.Cosslett, "Practical Electron Microscopy", Butterworths, Chapter 5, (1951).

4) P.B.Hirsh, A.Howie, R.B.Nicholson, D.W.Pashley \& M.J.Whelan, "Electron Microscopy of Thin Crystals", Butterworths, Chapter 1, (1965).

5) O.Scherzer, J.Appl.Phys., 20, 20 (1949) .

6) E.Ruska, in "Advances in Optical and Electron Microscopy", vol.1, R.Barer \& V.E.Cosslett Ed., Academic Press, 115 (1966).

7) Y.Fujiyoshi, Denken(Electron-Microsc.), 15, 72 (1980).

8) G.R.Grinton \& J.M.Cowley, OPTIK, 34, 221(1971).

9) J.M.Cowley, "Diffraction Physics", North-Holland, Chapter 13, (1975) .

10) J.M.Cowley, ibid., Chapter 4, (1975).

11) J.W.Goodman, "Introduction to Fourier Optics", McGrow-Hill, Chapter 5, (1968).

12) W.T.Cathey, "Optical Information Processing and Holography", John Wiley \& Sons, Chapter 5, (1974).

13) N.Uyeda \& K.Ishizuka, J.Electron Microsc., 23, 79 (1974). 
14) K.Iizuka, "Hikari-Kogaku", Kyoritsu, Japan, Chapter 5, (1977).

15) F.Thon, in "Electron Microscopy in Material Science", Ed. by U.Valdré, Academic Press, $562(1971)$.

16) K.-J.Hanszen, in "Advances in Optical and Electron Microscopy", vol.4, R.Barer \& V.E.Cosslett Ed., Academic press, 1 (1971).

17) H.P.Erickson, in "Advances in Optical and Electron Microscopy", vol.5, R.Barer \& V.E.Cosslett Ed., Academic Press, 163(1973).

18) P.B.Hirsh, A.Howie, R.B.Nicholson, D.W.Pashley \& M.J.Whelan, "Electron Microscopy of Thin Crystals", Butterworths, Chapter 4, (1965).

19) M.S.Isaacson, in "Principles and Techniques of Electron Microscopy", vol.7, M.A.Hayat Ed., Van Nostrand Reinhold, 1 (1977).

20) R.H.Wade \& J.Frank, Optik, 49, 81 (1977).

21) O.L.Krivanek, S.Isoda \& K.Kobayashi, J.Microsc., 111, 279 (1977) .

22) E.L.Thomas \& O.G.Ast, Polymer, 15, 37(1974).

23) C.J.D.Catto \& K.C.A.Smith, J.Microsc., 105, 223(1975).

24) R.C.Valentine, Labo.Invest., 14, 1334 (1965).

25) R.C.Valentine, in "Advances in Optical and Electron Microscopy", vol.l, R.Barer \& V.E.Cosslett Ed., Academic Press, $180(1966)$.

26) G.L.Jones \& V.E.Cosslett, Proc.7-th Int.Congr.EM, Grenoble, 349 (1970). 
27) H.Hashimoto, A.Kumao, S.Suzuki \& H.Yotsumoto, ibid., 351 (1970).

28) A.Fukami, M.Katoh \& K.Fukushima, ibid., 353(1970).

29) J.A.Aznarez \& F.Catalina, ibid., $355(1970)$.

30) V.Matricardi, G.Wray \& D.F.Parsons, Micron, 3, 526(1972).

31) G.C.Farnell \& R.B.Flint, J.Microsc., 97, 271 (1973).

32) M.V.King \& D.F.Parsons, Proc.4-th Int.Congr.HVEM, Toulouse, $75(1975)$

33) V.E.Cosslett, G.L.Jones \& R.A.Camps, Proc.3-rd Int.Conf.HVEM, Oxford, 147 (1974).

34) G.C.Farnell \& R.B.Flint, J.Microsc., 103, 319(1975).

35) K.Kobayashi \& N.Uyeda, Proc.8-th Int.Congr.EM, Canberra, vol.1, $264(1974)$

36) N.Uyeda, K.Ishizuka, Y.Saito, Y.Murata, K.Kobayashi \& M.O'hara, ibid., vol.1, $266(1974)$.

37) K.Ishizuka \& N.Uyeda, Bull.Inst.Chem.Res., Kyoto Univ., 53, $200(1975)$.

38) A.Kawaguchi, Thesis, Kyoto University, Chapter 1, (1979).

39) K.Ishizuka \& N.Uyeda, Acta Cryst., A-33, 740 (1977).

40) K.Ishizuka \& N.Uyeda, Bull.Inst.Chem.Res., Kyoto Univ., 55, 260 (1977).

41) M.A.O'Keefe \& P.R.Buseck, Proc.Symp.Chem.Phys.Minerals, University of Hawaii, 27 (1979).

42) J.M.Cowley, "Diffraction Physics", North-Holland, Chapter I, $19(1975)$ 
Chapter 2 : Morphology of PPX single crystals

The crystal structure of PPX was first reported in 1953 by Brown \& Farthing who discovered two crystalline modifications of $\alpha$ and $B$, corresponding to the low and high temperature modifications, respectively. In 1966, Niegisch found the presence of a new transition at $270^{\circ} \mathrm{C}$ through differential thermal analysis. This transition appears uniquely in the polymer prepared from di-p-xylylene ( see Fig.2-1). He also studied the morphology of both $\alpha$ and $\beta$ single crystals of PPX grown from $\alpha$-chloronaphthalene solution. Kajiura et al ${ }^{3}$. has confirmed by $x$-ray measurements that these two single crystals correspond to $\alpha$ - and $\beta$-forms of bulk materials observed by Brown \& Farthing. Kajiura et al. also investigated the anealing effects (the temperature dependence of the long periods) of $B$-form single crystals by x-ray diffraction. 


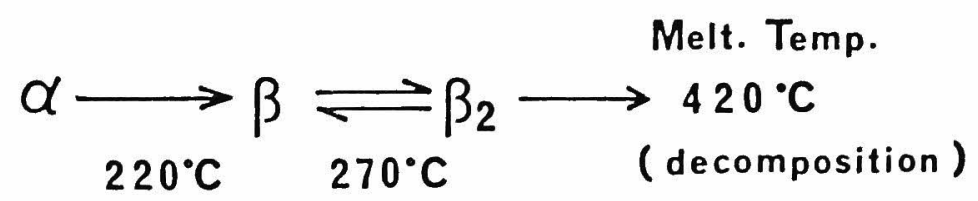

Fig.2-1 Transition temperatures of PPX crystals.

$(\text { Niegisch })^{2}$

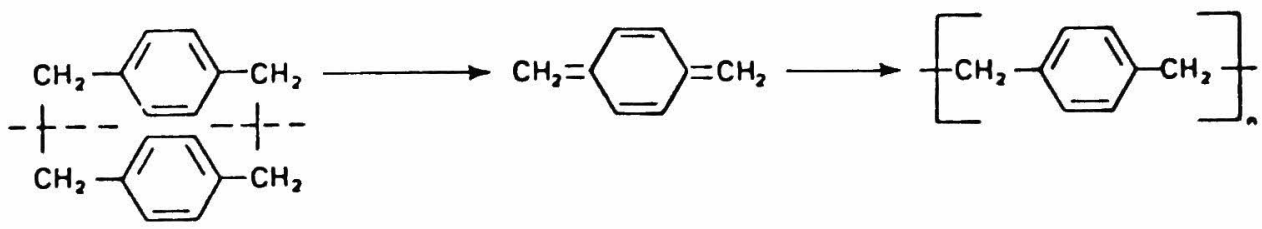

Di-para-xylylene

(Dimer)

1) Vaporize

$\sim 175^{\circ} \mathrm{C}$.

$\sim 1$ TORR para-xylylene

(Monomer)

2) Pyrolize

$-680^{\circ} \mathrm{C}$.

-0.5 TORR
Poly(para-xylylene)

(Polymer)

3) Deposition

$25^{\circ} \mathrm{C}$.

0.1 TORR

Fig.2-2 Diagram of the Parylene process. 


\begin{abstract}
Miles \& Gleiter studied the molecular mechanism of the $\alpha \rightarrow \beta$ phast transition of PPX single crystal by transmission electron microscopy using the heating specimen holder.
\end{abstract}

2-1 Morphology of PPX single crystals

The PPX sample used is Parylene N (molecular weight is approximately 500,000); the product of Union Carbide Coxporation ir U.S.A. and provided through Tomoe Engineering Co. Ltd. (Tokyo, Japan). Parylene N ( a linear high polymer of PPX) is produced by vacuum vapor-phase pyrolysis of di-p-xylylene according to the Gorham's method. The schematic diagram of the preparation process is shown in Fig.2-2. The end products of PPX film are transparent and colorless, coherent, and pinhole-free in the thickness range from a few hundred \& to many hundred $\mu \mathrm{m}$. These are some of the characteristics which make PPX a useful coating or encapsulating material for complex objects such as electronic circuit boards.

The morphological forms depend on the crystallization condition, especially on crystallization solvents according to Kajiura et al. Here we used $\alpha$-chloronaphthalene as a solvent after Niegisch. Parylene $N$ film was dissolved in $\alpha$-chloronaphthalene by heating in a salt bath. The solution (0.05\%) was cooled to $210^{\circ} \mathrm{C}$, held at this temperature overnight and then cooled to the room 


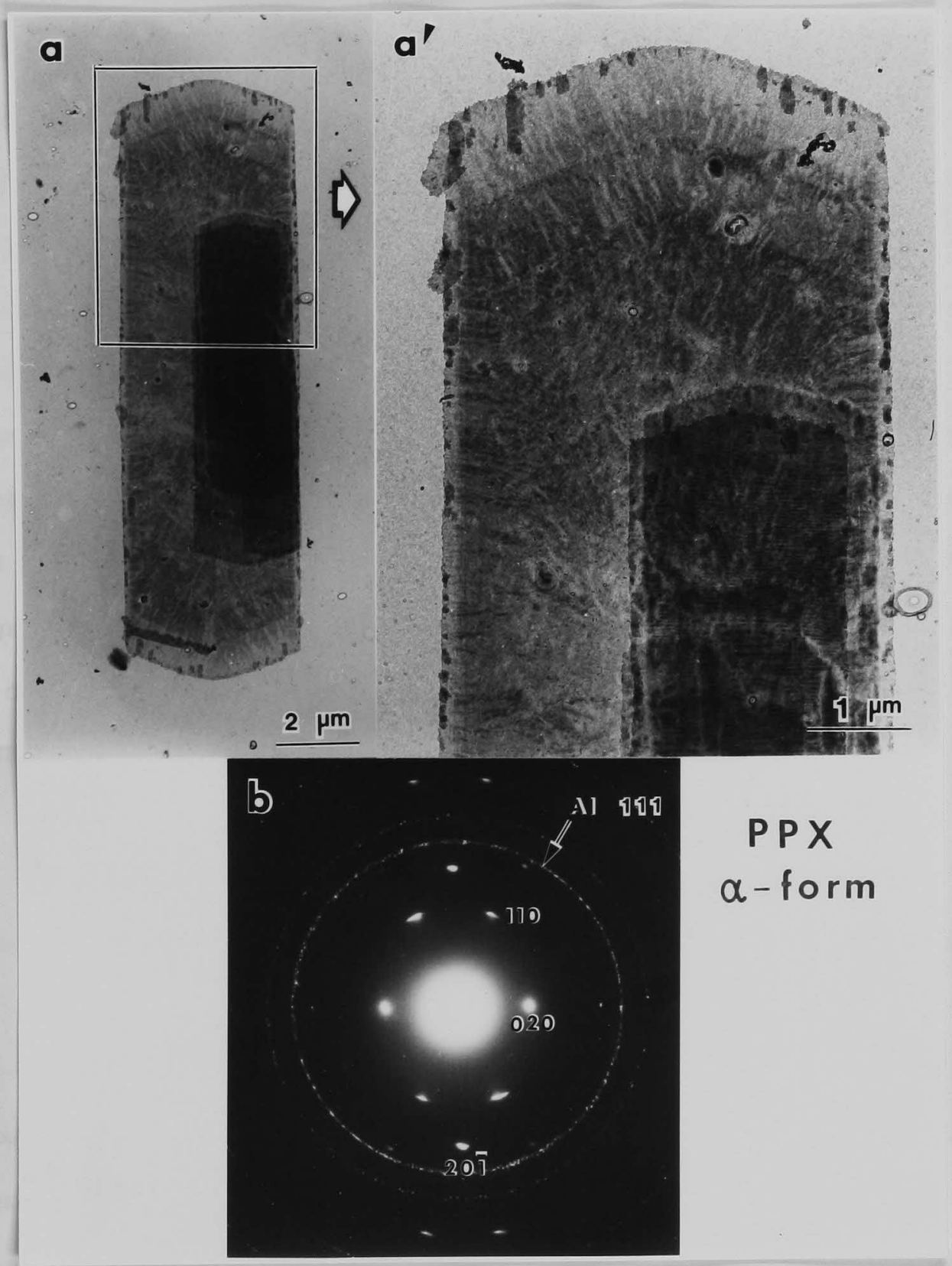

Fig.2-3 PPX $\alpha$-form single crystal (a);

$\left(a^{\prime}\right)$ : enlarged photograph of the enclosed part in (a),

(b) : electron diffraction pattern of an $\alpha$-form single crystal on Al support-film. 


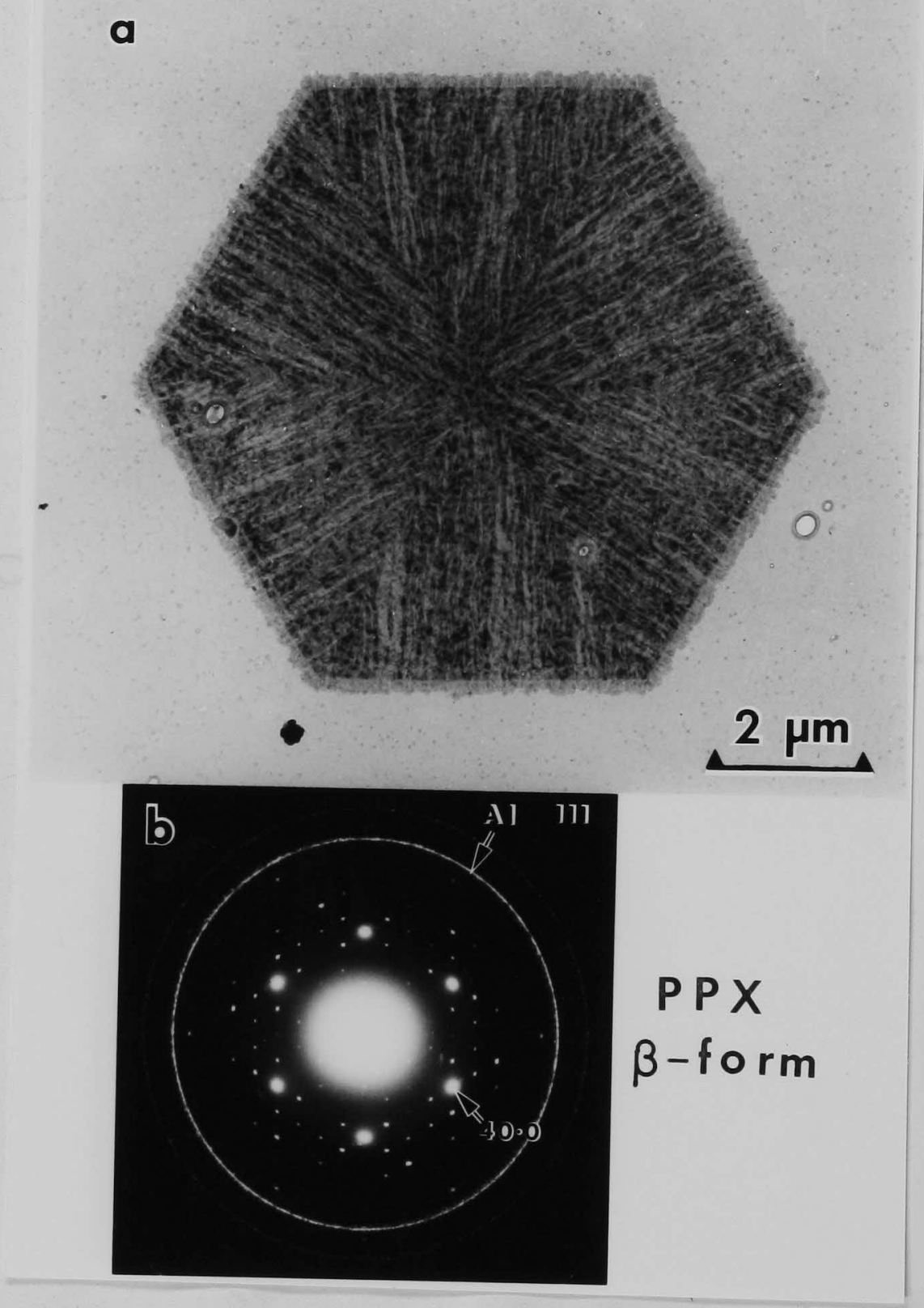

Fig.2-4 PPX $\beta$-form single crystal (a) and electron diffraction pattern (b) of a $\beta$-form single crystal on Al supportfilm. 
temperature. All the treatments were taken under a nitrogen atmosphere to prevent polymer degradation in the presence of oxygen. The polymer is also reported insoluble in the presence of oxygen. Two entirely different types of single crystals ( in fact, they are so-called "mosaic crystals", developed from the solution under the experimental conditions described above. Here Fig.2-3 shows a rectangular crystal attributed to the $\alpha$-modification and Fig.2-4 a hexagonal crystal attributed to the $\beta$-modification, together with respective electron diffraction patterns. The crystal thickness of the $\alpha$-form was $\sim 120 \AA$, and that of the $\beta$-form was $280 \AA$. Electron micrographs and selected-area electron diffraction photographs in this chapter were all taken on FUJI electron-microscopic films (FG) with JEM-7A using an accelerating voltage of $80 \mathrm{KV}$.

\section{2-1-1 The $\alpha$-modification of PPX single crystal}

In the case of the $\alpha$-modification of PPX single crystal, two types of diffraction patterns were obtained, corresponding to a single-layer or multi-layer crystal. Figure 2-5 shows both kinds of selected area electron diffraction patterns and their double-exposed micrographs with a selected area aperture. Kubo \& Wunderlich reported the unit cell dimensions of the $\alpha$-modification, and Iwamoto \& Wunderlich analyzed the crystal structure of this modification based on the result by Kubo \& Wunderlich. This result is cited in Fig.2-6 and Table 2-1. As shown in Fig.2-5, a 


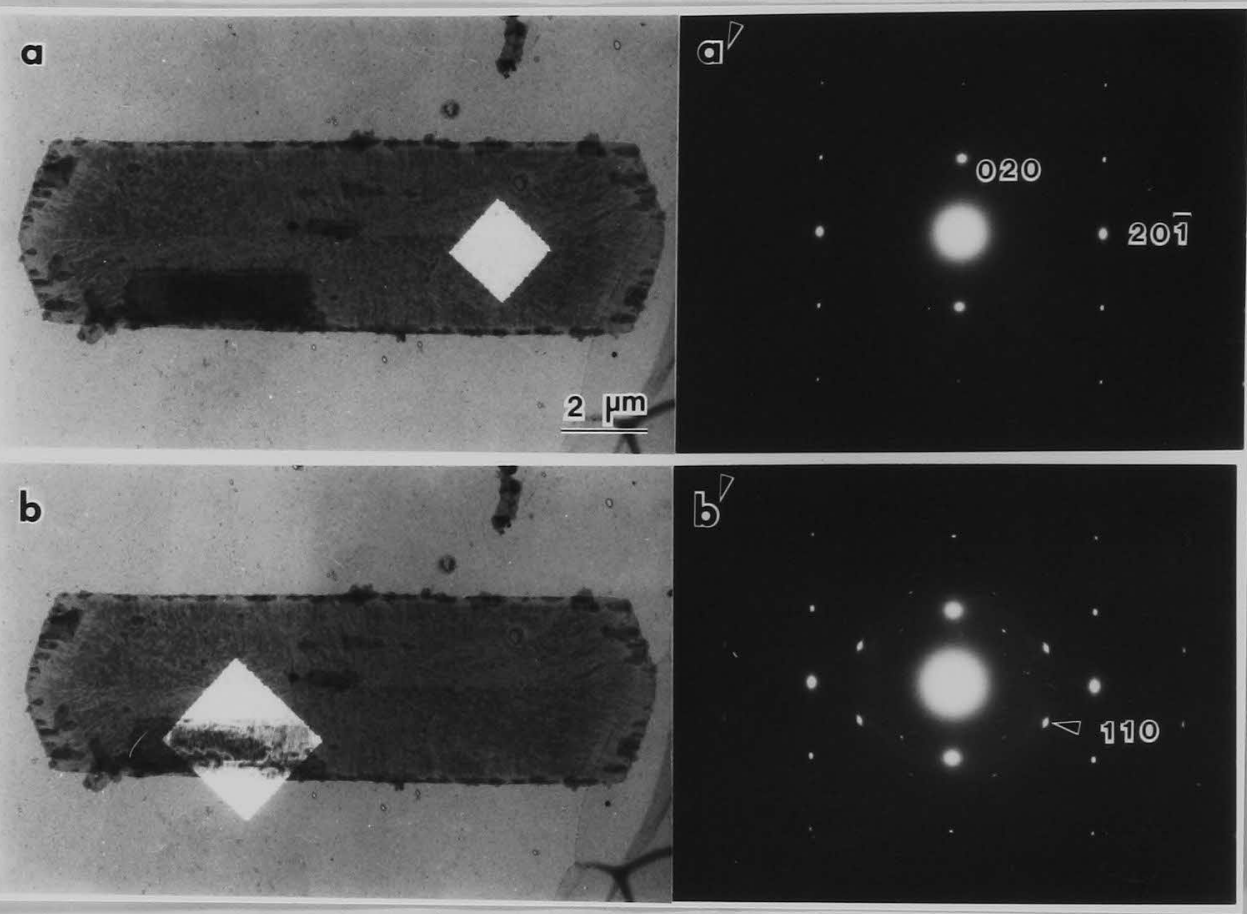

Fig.2-5 PPX $\alpha$-form single crystal and corresponding selectedarea electron diffraction patterns.

The squares in (a) and (b) show the selected-area aperture positions corresponding to $\left(a^{\prime}\right)$ and $\left(b^{\prime}\right)$, respectively. 
single-layer crystal yields the diffraction pattern corresponding to <102> incidence of electron beam. A multi-layer crystal yields the (110) reflections corresponding to <001> incidence of electrons as well as the diffraction pattern of <102> incidence. That is also confirmed with JEM-500 in which much smaller selected-area apertures are available than in JEM-7A. Accordingly it is concluded that the chain axis in the basal layer is not perpendicular to the crystal end-surfaces ( namely, not parallel to the direction of the incident electron beam ), but the chain axis in other layers is parallel to the incident beam direction. The parallel fringes seen in the multi-layer part in Fig.2-3 are moiré-image interference fringes. The radial stripes seen in the single basal layer are due to diffraction contrast, indicating that there exist 6 sectors as shown 6 by a tent-like structure in Fig.2-7, in prior to its deposition on the specimen support-film. Moiré fringes and radial stripes can also be observed in the dark-field electron micrograph in Fig.2-8. These fringes and stripes are to be observed as far as the crystal is living under electron irradiation.

2-1-2 The $B$-modification of PPX single crystal

Most of the PPX $\beta$-form single crystals have nearly flat and regular-hexagonal shapes as shown in Fig.2-4, but some exhibit irregular forms ( see Fig.2-9) while yielding a hexagonal diffraction pattern. The electron diffraction pattern of the $B$-form 
Table 2-1

Atomic Coordinates and Temperature Factors in the $\alpha$-Form of PPX

\begin{tabular}{lccrc}
\hline Atom & $\mathbf{x}$ & $\mathrm{y}$ & $\mathrm{z}$ & $\mathrm{B}\left(\AA^{2}\right)$ \\
\hline C1 & -0.1546 & 0.0 & -0.0355 & 5.0 \\
C2 & -0.0734 & 0.0 & 0.2454 & 5.0 \\
C3 & -0.0367 & 0.1131 & 0.3722 & 5.0 \\
& \multicolumn{4}{c}{ ( Iwamoto \& Wunderlich ) }
\end{tabular}
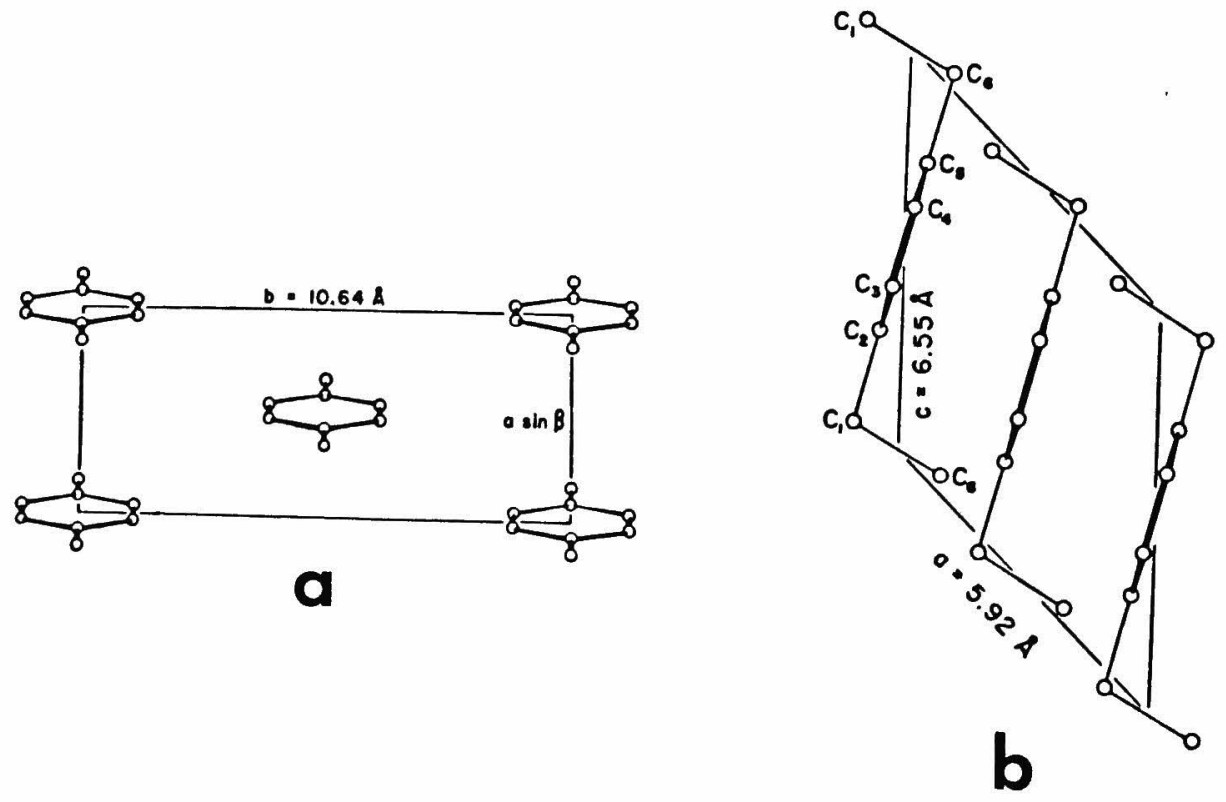

Fig.2-6 Crystal structure of the PPX $\alpha$-form crystal projected (a) along the c-axis and (b) onto the ac-plane. space group : monoclinic, $\mathrm{C} 2 / \mathrm{m}$

lattice constants : $\begin{aligned} & a=5.92 \AA, b=10.64 \AA, c(\text { fiber axis) }=6.55 \AA \\ & \beta=134.7^{\circ}\end{aligned}$ (Iwamoto \& Wunderlich) ${ }^{7}$ 


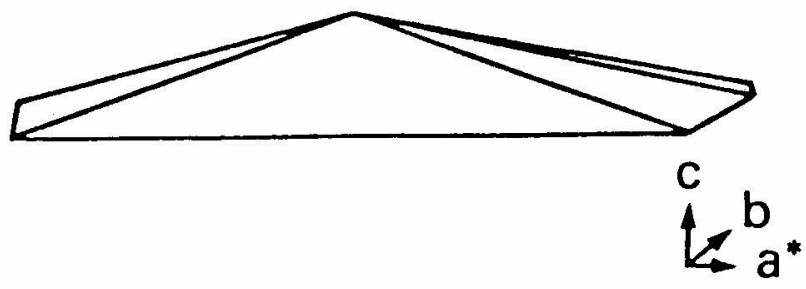

Fig.2-7 Schematic representation of the tent-like structure of a PPX $\alpha$-form single crystal.

(Kubo \& Wunderlich) ${ }^{6}$

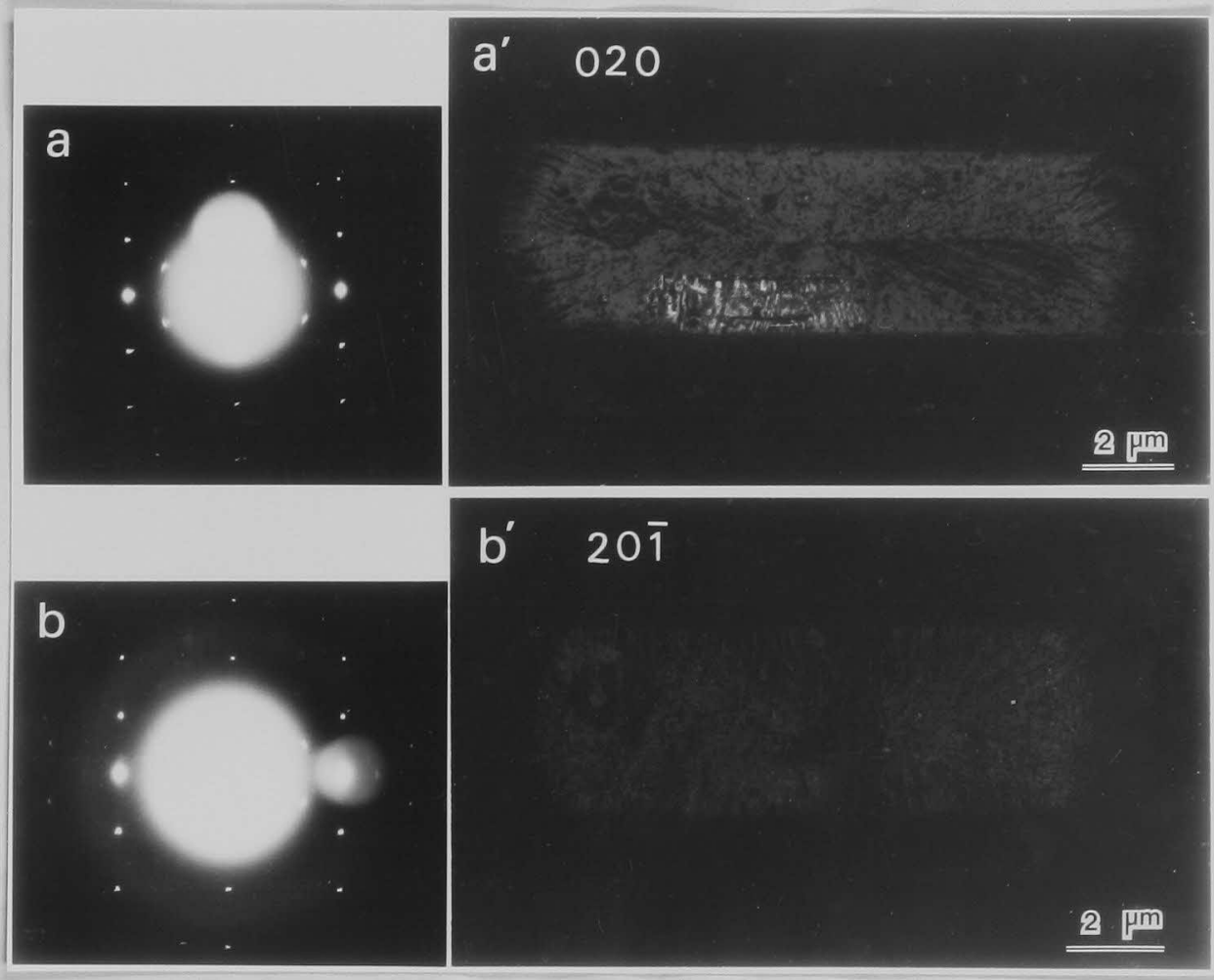

Fig.2-8 Dark-field images of a PPX $\alpha$-form single crystal using the 020 reflection or the $20 \bar{I}$ reflection. The objective aperture positions corresponding to (a') and (b') are shown in electron diffraction photographs (a) and (b), respectively. 


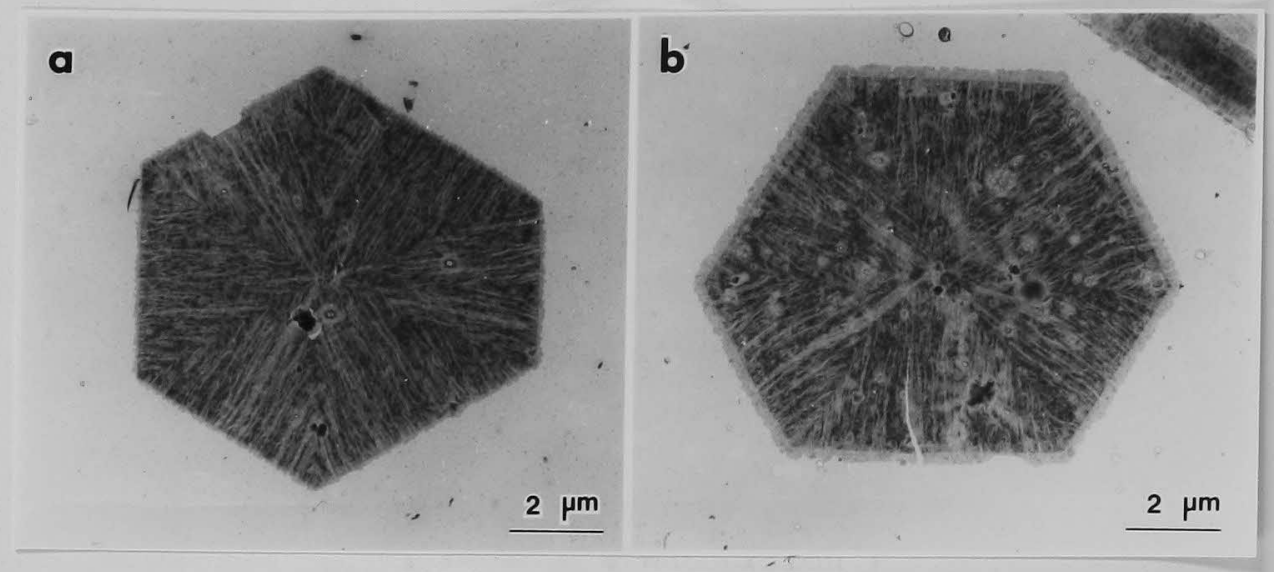

Fig.2-9 PPX $\beta$-form single crystals of irregular hexagonal shape.

a

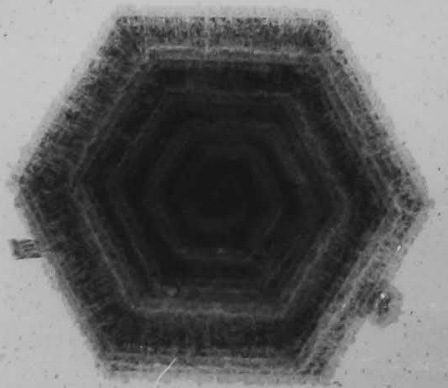

$2 \mu \mathrm{m}$ b

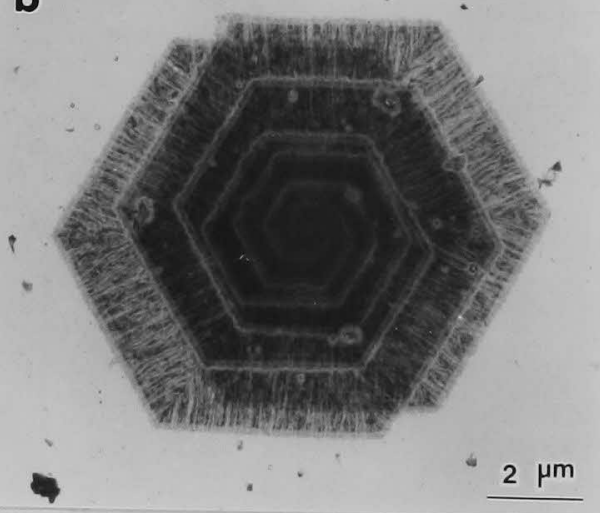

Fig.2-10 Examples of spirally grown single crystals of the PPX $\beta$-form.

(a) : typical spiral growth,

(b) : double spiral growth with the same sign. 
single crystal shows no systematic absences of reflections as 2 reported by Niegisch and has no symmetry planes ( see Fig.2-4(b) or see Fig.2-14(a') ). This diffraction pattern is characterized by 6-fold rotational symmetry. These facts were confirmed by inspecting more than 100 photographs of the electron diffraction patterns of the $\beta$-form single crystals. Spirally grown multi-layer crystals are observed on the $\beta$-form as was the case of polyethylene, polyoxymethylene, and so on. Typical crystals are shown in Figs.2-10(a) and (b). A twin-like crystal was also found as shown in Fig.2-11. $O X$ and $O Y$ in Fig.2-11(b) denote the boundaries in this crystal, which are defined by radial stripes. The radial stripes which are seen in all the $\beta$-form crystals are due to diffraction contrast as observed in $\alpha$-forms, and they indicate that there exist six (10.0) sectors in the $\beta$-form single crystal. All the sectors yield the identical diffraction pattern. The dark-field electron micrograph is shown for reference in Fig.2-12. In Figs.2-8 and 2-12 it is also confirmed that the radial stripes which are seen in bright-field micrographs (Figs.2-3 and 2-4) of $\alpha$ - and $\beta$-form single crystals are due to diffraction contrast. The stripes disappear under strong electron irradiation. Unlike the $\alpha$-form, it is characteristic of the $\beta$-form that the multi-layer crystals never yield moiré fringes. Niegisch has estimated the hexagonal unit cell dimension ( $a=20.52 \AA, c$ (fiber axis $=6.58 \AA$, and concluded that the molecules are all parallel to 


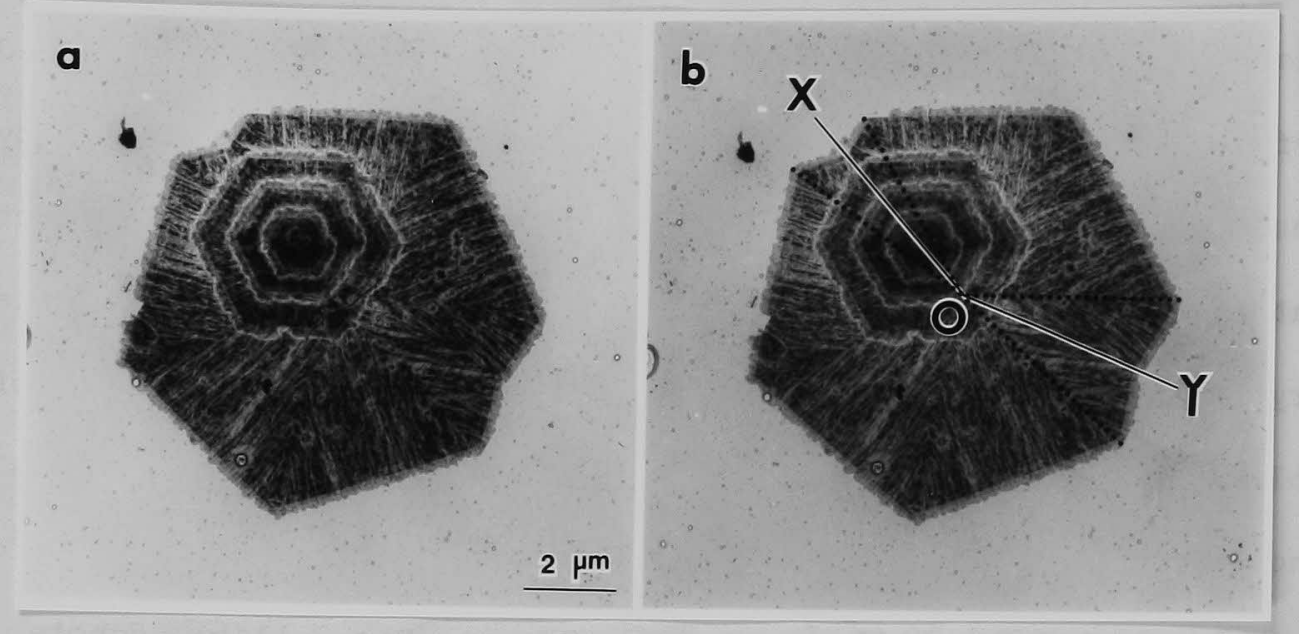

Fig.2-11 Twin-like single crystal of the PPX $\beta$-form. $O X$ and $O Y$ in (b) indicate the boundaries, which can be defined by radial stripes due to diffraction contrast.

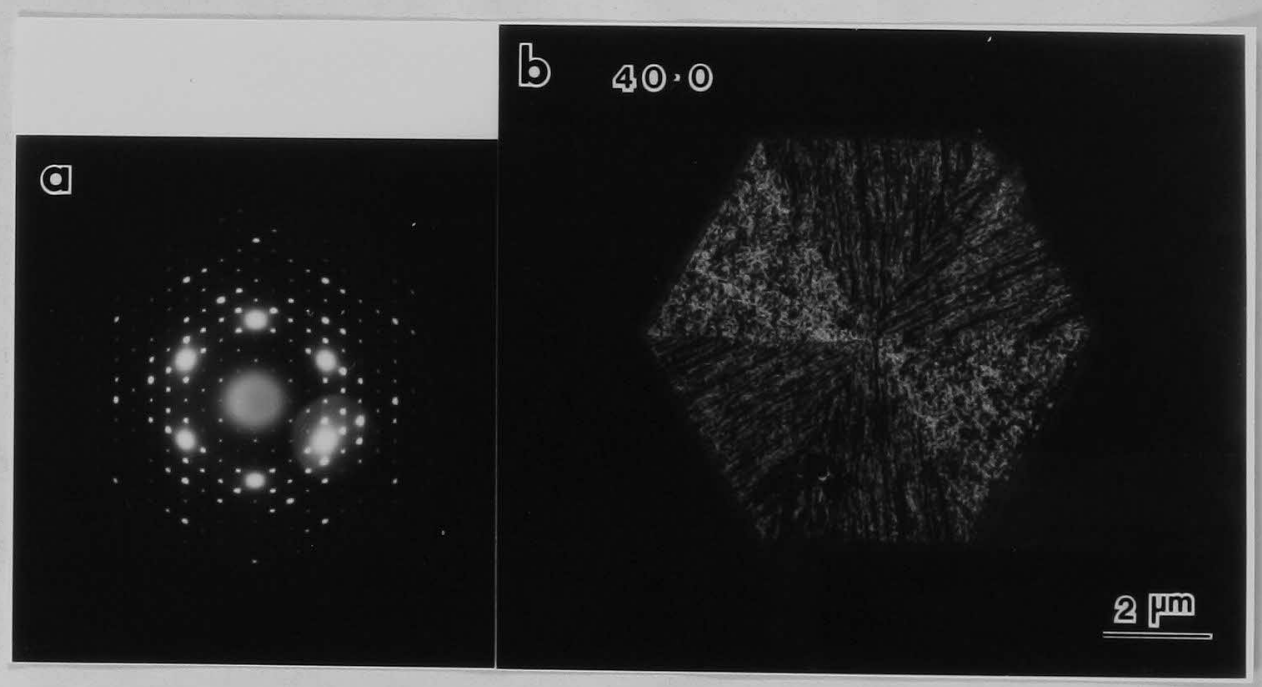

Fig.2-12 Dark-field image of a PPX $\beta$-form single crystal using the 40.0 and its neighbouring reflections. The objective aperture position corresponding to (b) is shown in the electron diffraction photograph (a). 
the c-axis, which is perpendicular to the single crystal end-surface. The detailed crystal structure is not yet known.

2-2 Effects of electron irradiation on PPX single crystals

In general, polymer crystals (such as polyethylene, polyoxymetylene, and so on) are vulnerable to electron irradiation, and the molecular chains are crosslinked to become amorphous or are 11-22

decomposed in a short time under applying electrons. Both $\alpha$ - and $\beta$-forms of PPX crystals are, however, fairly strong as compared with polyethylene. Figures 2-13 and 2-14 show the morphological changes of PPX $\alpha$ - and $\beta$-form single crystals due to $80 \mathrm{KV}$ electron irradiation with their electron diffraction patterns, respectively. In the case of the $\alpha$-form ( see Fig.2-13), the radial stripes in the single basal layer (due to diffraction contrast) disappear at first by irradiation. The parallel fringes (viz. moiré fringes) in the multi-layer part ( see Fig.2-3( $\left.a^{\prime}\right)$ ) also vanish. The $\beta$-modifications yield no moiré-image interference fringes, as described in previous section (2-1). Figure 2-14 indicates that the radial stripes due to diffraction contrast disappear partially at first and then the area of no stripes observed spreads over the whole crystal, till entire stripes have gone. The crystalline reflections disappear from higher orders with increasing doses in 
the case of the $\alpha$-form ( see Fig.2-13). However, in the case of the $\beta$-form, the reflections of odd-number indices such as $(50.0)$, (41.0) and so on, disappear at first ( see Fig.2-14(c') ), and the reflections having the indices of an integral multiple of 4 , such as $(40.0),(44.0)$ and so on, remain (see Fig.2-14(d') ). It seems from Fig.2-14(d') that molecular chains are packed in the crystal with hexagonal symmetry where only one chain segment is contained in a unit cell, at such a irradiation dose. Then all the crystalline reflections disappear at last.

Disappearance of the stripes due to diffraction contrast and/or of moiré fringes as well as vanishing of the crystalline reflections denotes the "death" of the crystals. The effects of electron irradiation on polyethylene crystals were discussed by Kobayashi \& 11,14

sakaoku. They illustrate that crosslinking between adjacent molecular chains will take place in polymer crystals under electron irradiation ( see Fig.2-15). In the case of polyoxymethylene, the decomposition follows after crosslinking and the crystal itself 22 disappears. As shown in Figs.2-13 and 2-14, the external views of both PPX $\alpha$ - and $\beta$-form single crystals are unchanged, suggesting that crosslinking takes place in the PPX crystals under electron irradiation without decomposition. A lucid explanation of the irradiation effects on polymer crystals can be expected in the near future, if we carefully observe the change in electron micrograph as well as that in electron diffraction. 


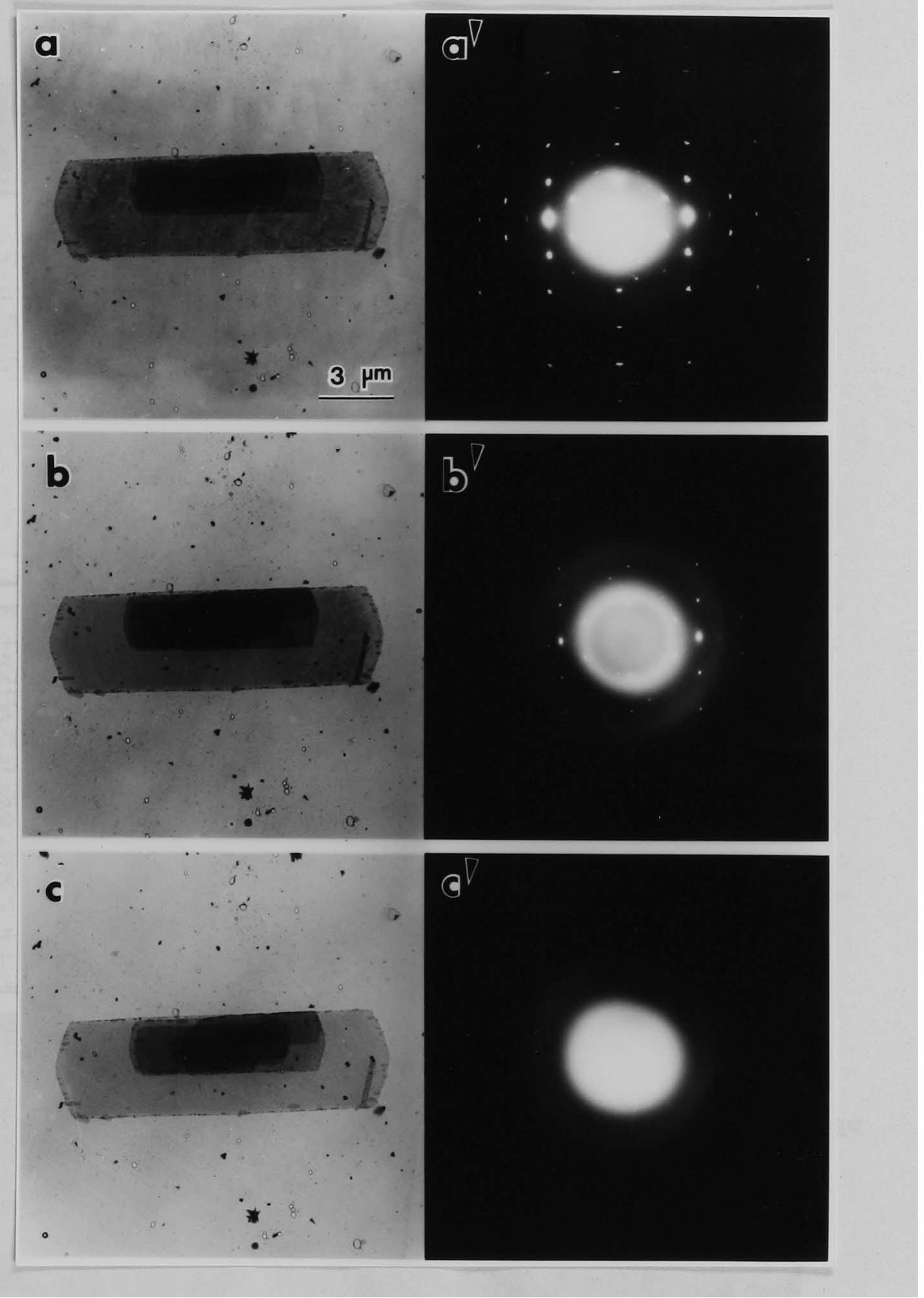

Fig.2-13 Morphological changes of a PPX $\alpha$-form single crystal due to $80 \mathrm{KV}$-electron irradiation.

(a')-(c') are electron diffraction patterns corresponding to $(a)-(c)$, respectively. 


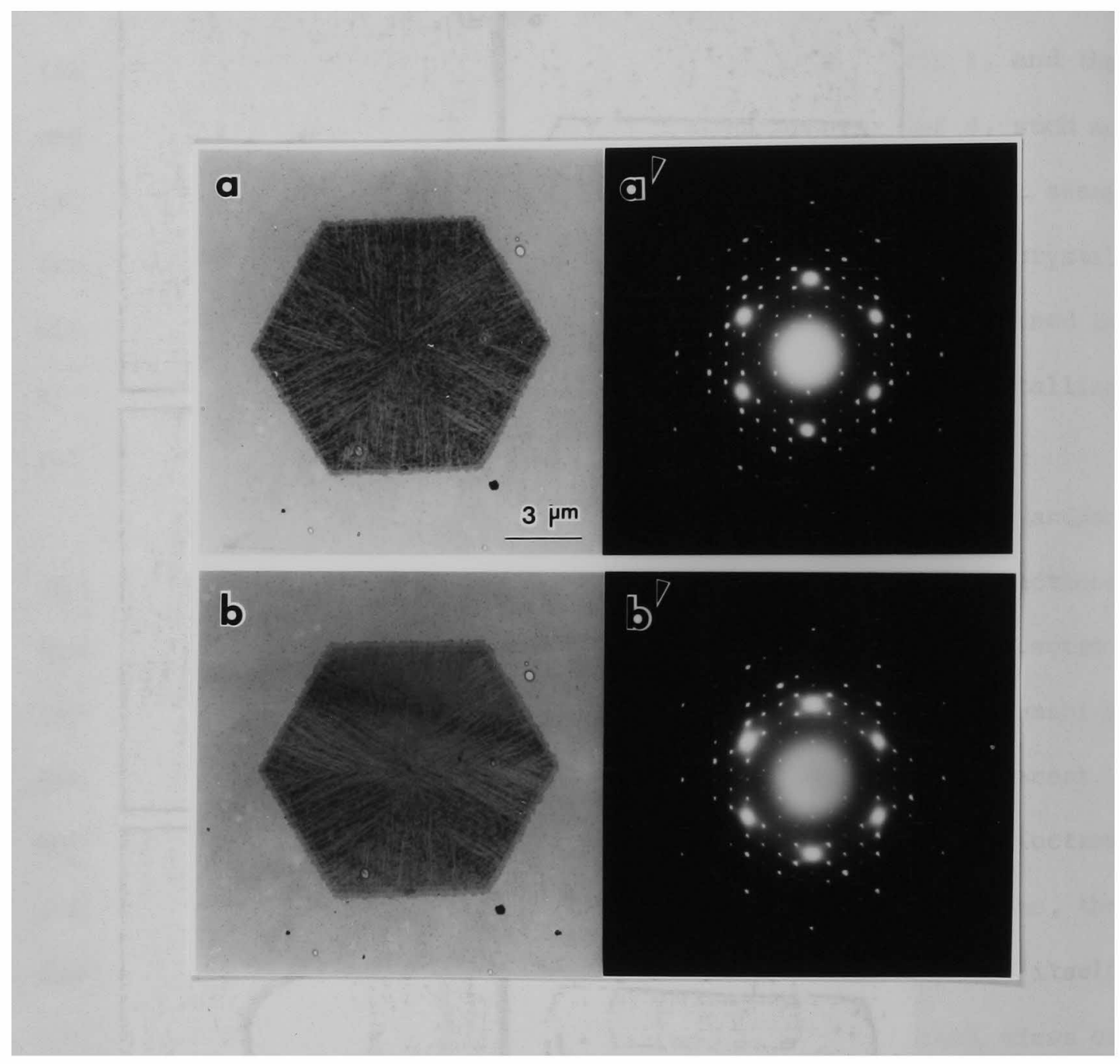

Fig.2-14 Morphological changes of a PPX $\beta$-form single crystal due to $80 \mathrm{KV}$-electron irradiation. $\left(a^{\prime}\right)-\left(e^{\prime}\right)$ are electron diffraction patterns corresponding to $(a)-(e)$, respectively. 


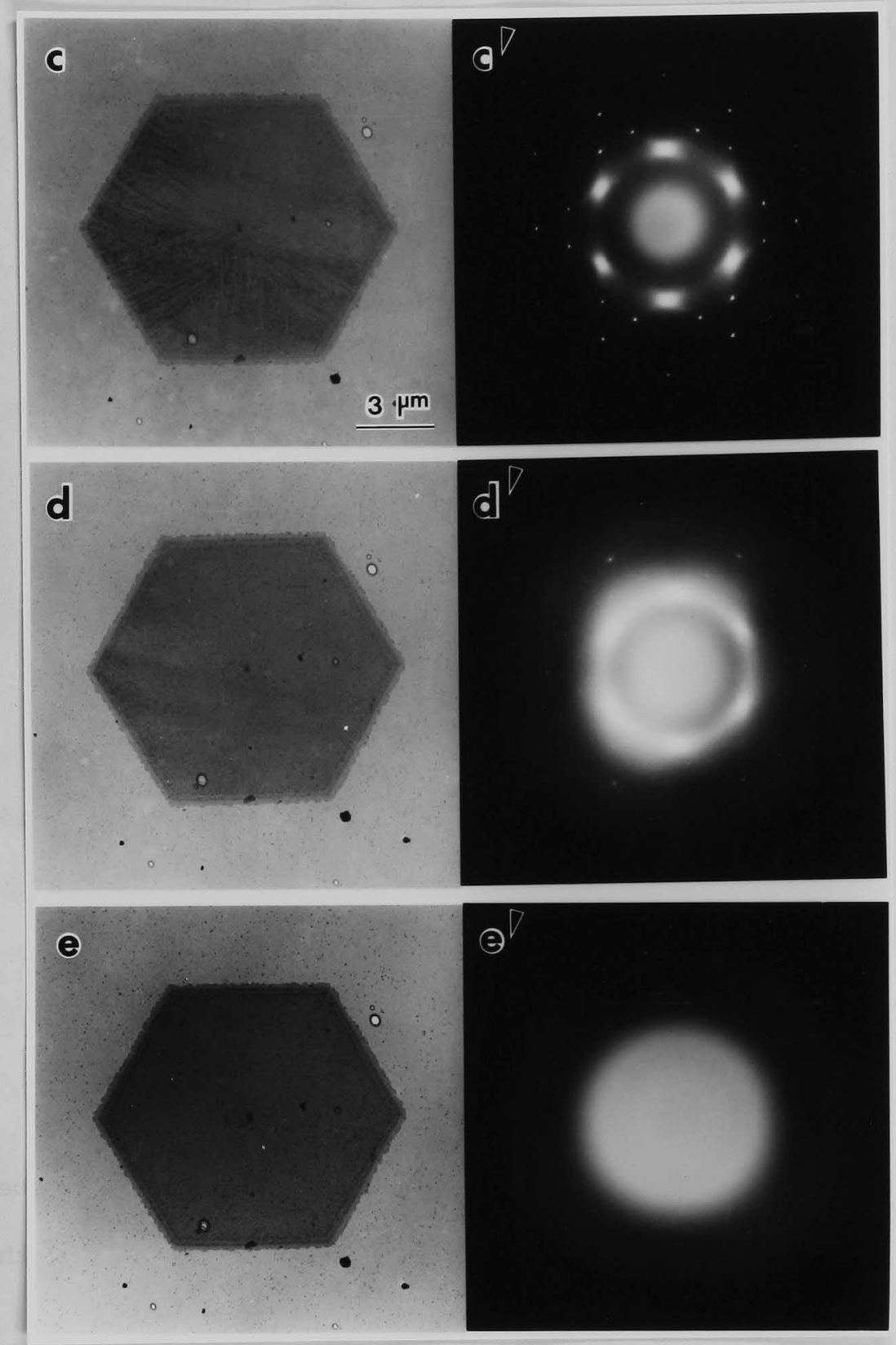

Fig.2-14 (continued) 


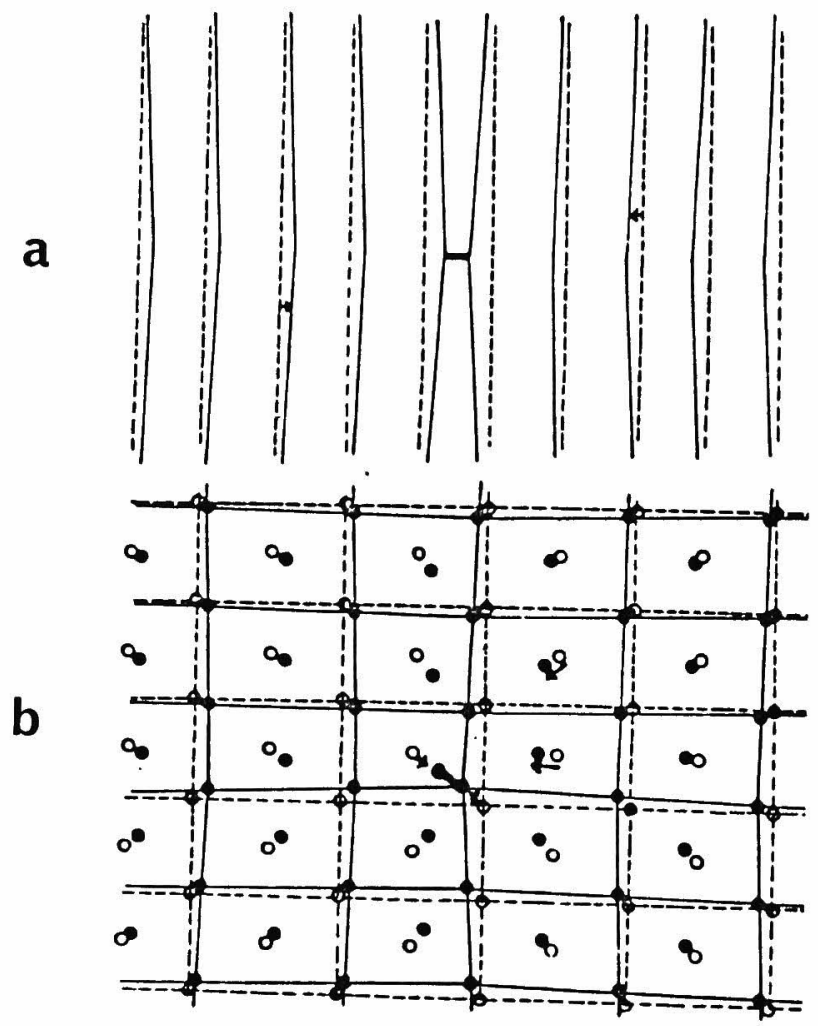

Fig.2-15 Distorted crystal lattice of PE with a crosslink between molecular chains due to electron irradiation. Side view along the b-axis (a) and projection on the ab-plane (b). Dotted lines represent original (undistorted) lattice.

(Kobayashi \& Sakaoku) $)^{11}$ 
References

1) C.J.Brown \& A.C.Farthing, J.Chem.Soc., 1953, 3270 (1953).

2) W.D.Niegisch, J.Appl.Phys., 37, 4041 (1966).

3) A.Kajiura, M.Fujii, K.Kikuchi, S.Irie \& H.Watase, Kolloid-Z., 224, 124 (1968).

4) M.Miles \& H.Gleiter, J.Macromol.Sci.-Phys., B15, 613 (1978).

5) W.F.Gorham, J.Polymer Sci.:A-1, 4, 3027(1966).

6) S.Kubo \& B.Wunderlich, Makromol.Chem., 162, I(1972).

7) R.Iwamoto \& B.Wunderlich, J.Polymer.Sci.-Phys., 11, 2403 (1973).

8) K.Kobayashi, in "Kobunshi no Bussei", Kagakudojin, Kyoto, (1962).

9) P.H.Geil, "Polymer Single Cristals", Interscience-Pub., (1963)

10) B.Wunderlich, "Macromolecular Physics", vol.1, Academic-Press, (1973)

11) K.Kobayashi \& K.Sakaoku, Bull.Inst.Chem.Res., Kyoto Univ., $\underline{42}, 473(1964)$.

12) H.Orth \& E.W.Fischer, Makromol.Chem, 88, 188 (1965).

13) L.Reimer, Labo.Invest., 14, 1082 (1965).

14) K.Kobayashi \& K.Sakaoku, Labo.Invest., 14, $1097(1965)$.

15) K.Kobayashi \& M.O'hara, Proc.6-th Int.Congr.EM, Kyoto, $579(1966)$.

16) H.Kiho \& P.Ingram, Makromol.Chem., 118, 45 (1968).

17) D.T.Grubb \& G.W.Groves, Phil.Mag., 24, 815(1971). 
18) D.T.Grubb, A.Keller \& G.W.Groves, J.Materials Sci., I, $131(1972)$

19) J.Petermann \& H.Gleiter, Kolloid-Z, 251, 850 (1973).

20) A.Kawaguchi, Bull.Inst.Chem.Res., Kyoto Univ., 57, 206 (1979).

21) G.Ungar, D.T.Grubb \& A.Keller, Polymer, 21, 1284 (1980).

22) D.C.Bassett, Phil.Mag., 10, 595 (1964).

23) W.D.Niegisch, J.Appl.Phys., 38, 4110 (1967). 
hapter 3 : High resolution electron microscopy of PPX single crystals

Using the high resolution electron microscope (HREM), the direct observation of crystal structure by interference of many beams diffracted from a thin crystalline specimen has been developed for various types of inorganic materials which are strong against electron irradiation. Hashimoto et al. observed the image of gold single crystal with circular fine structure ( it is due to the nucleus and the electron cloud in the specimen atoms) under "Aberration Free Focus" (AFF) condition. Others also reported that they observed isolated heavy atoms by means of a conventional EM.

Since Uyeda et al. observed the molecular images of hexadecachlorocopper phthalocyanine in a crystal, the molecular images of several organic crystals which are relatively less sensitive to electrons have been also reported. In the case of 
radiation-sensitive polymer crystals, the high resolution microscopy was used for one-dimensional lattice imaging of $\mathrm{PPX}$ or 34-36

poly (p-phenylene terephthalamide) crystals.

As described in Introduction, Bassett \& Keller succeeded i. taking a dark-field electron micrograph showing the direct image of the $18 \AA$ (10.0) lattice spacing of a PPX $\beta$-form single crystal. Wt have taken notice of above-mentioned fact and have attempted to 37,38
resolve individual chains of PPX with HREM (JEM-500: accelerating voltage, $500 \mathrm{KV}$; spherical aberration coefficient, $\mathrm{C}_{\mathrm{S}}=1.06 \mathrm{~mm}$; resolving power, $1.4 \AA$ ) in our Institute.

3-1 The effect due to $500 \mathrm{KV}$ electron irradiation

As described in Chapter 2, most of the polymer crystals art less resistant against electron irradiation, and are crosslinked tc become amorphous or are decomposed in a short time under applyinc electrons. In this section, first we discuss quantitatively the effects of $500 \mathrm{KV}$-electron irradiation on PPX single crystals. The changes of the lattice spacings by irradiation of $500 \mathrm{KV}$-electrons are plotted in Fig.3-1(a) and (b). These measurements were carried out with the PPX single crystals mounted on an aluminum thin support-film using the diffraction rings of aluminum as the reference. The KODAK $x$-ray films $(A A-5)$, which are several times 

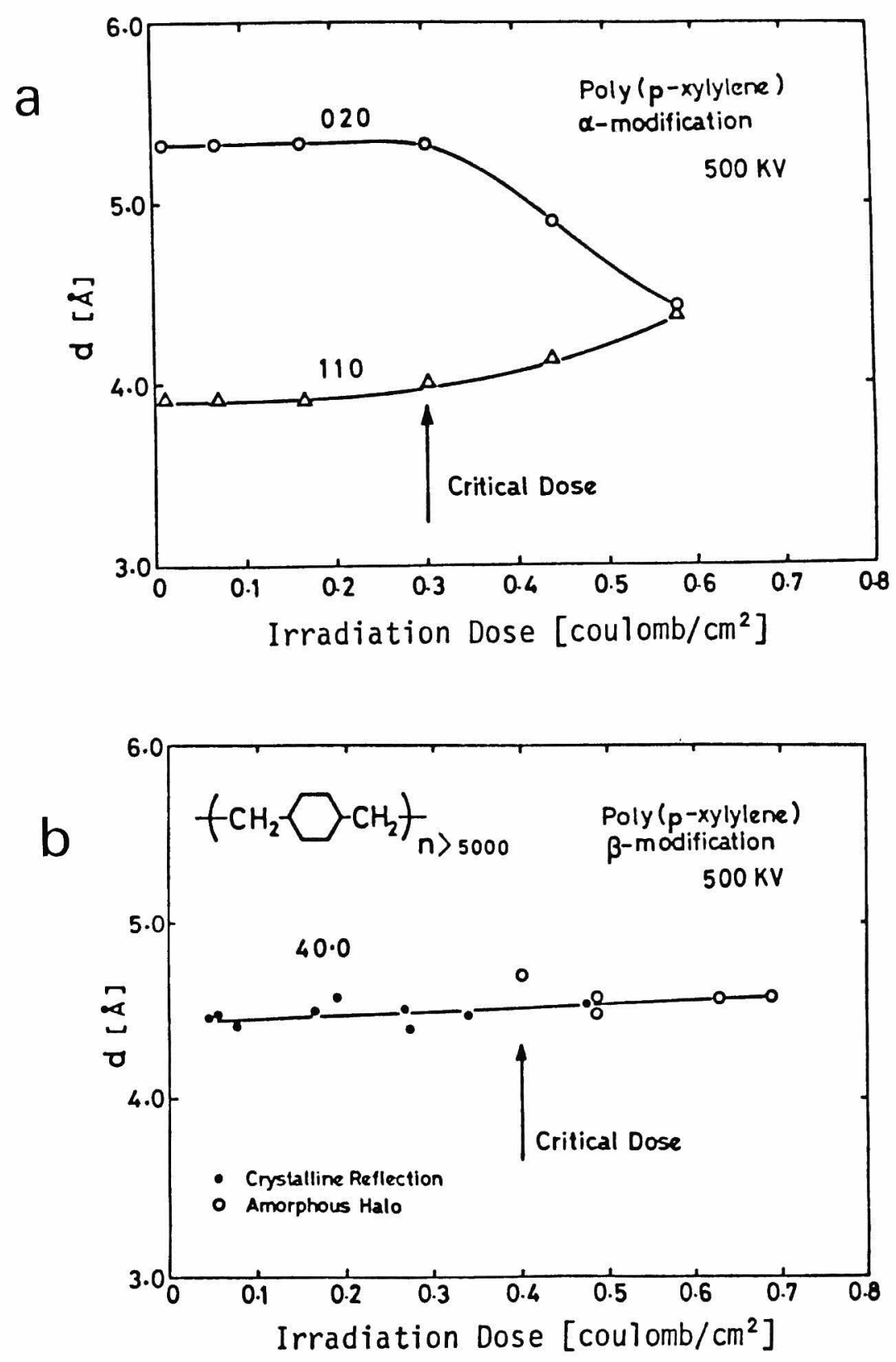

Fig.3-1 Changes of the lattice spacings of PPX single crystal due to $500 \mathrm{KV}$-electron irradiation.

(a) ; change of (020) and (110) spacings of a PPX $\alpha$-form single crystal.

(b) ; change of $(40.0)$ spacing of a PPX $\beta$-form single crystal. 
Table 3-1

Total End Point Doses of various organic crystals and calculated limits of detectability using Eq.(3-1)

\begin{tabular}{|c|c|c|c|c|c|}
\hline Specimen & $\left.\stackrel{\mathrm{Q}}{\mathrm{TEPD}}^{\mathrm{E}} \mathrm{e}^{2} \mathrm{mb} / \mathrm{cm}^{2}\right)^{-}$ & $\begin{array}{l}\text { Electron } \\
\text { energy } \\
\quad(\mathrm{KeV})\end{array}$ & Method De & $\begin{array}{l}\text { detecta- } \\
\text { bility } \\
(\AA)\end{array}$ & Refs. \\
\hline 1-Valine & 0.001 & 80 & ED & 126 & 73) \\
\hline Leucine & $.0015-0.002$ & $(60)$ & ED & 89 & $57), 58)$ \\
\hline Adenosine & 0.01 & 80 & ED & 40 & 73) \\
\hline Stearic acid & $.002-0.003$ & $(60)$ & ED & 73 & $57), 58)$ \\
\hline \multirow[t]{2}{*}{ Paraffin } & $.003-0.005$ & $(60)$ & $E D$ & 57 & 57), 58) \\
\hline & 0.01 & 100 & $\mathrm{ED} / \mathrm{C}$ & 40 & 59) /60) \\
\hline POM & $.006-0.01$ & 100 & $E D$ & 40 & $43), 44) / 61)$ \\
\hline \multirow[t]{4}{*}{$\mathrm{PE}$} & 0.002 & 75 & $\operatorname{LM}(\sim 5 \%)$ & 89 & 63) \\
\hline & $.006-0.01$ & 100 & $\mathrm{ED}$ & 40 & 43), 44) \\
\hline & $.006-0.01$ & 100 & ED & 40 & 47),61) \\
\hline & 0.01 & $80 / 100$ & $E D / C$ & 40 & $70) / 62)$ \\
\hline PEO & 0.003 & $200-300$ & ED & 73 & $43), 44)$ \\
\hline Nylon 6 & 0.01 & 100 & ED & 40 & 43), 44) \\
\hline $\begin{array}{c}\text { Poly-4-methyl- } \\
\text { pentene } 1\end{array}$ & 0.003 & 100 & ED & 73 & 61) \\
\hline Cellulose I & 0.04 & 100 & $E D$ & 73 & 64) \\
\hline PPTA (Kevlar) & 0.2 & 100 & ED & 8.9 & 36) \\
\hline $\mathrm{PPX}$ & 0.4 & 500 & ED & 6.3 & This work \\
\hline \multirow[t]{2}{*}{ Anthracene } & $0.06-0.08$ & $(60)$ & ED & 14 & $57), 58)$ \\
\hline & 0.12 & 100 & $E D$ & 11 & $65)$ \\
\hline \multirow[t]{2}{*}{ Tetracene } & $0.04-0.15$ & 100 & ED & 10 & 65) \\
\hline & $0.13-0.17$ & 100 & ED & 9.7 & $57), 58) / 59)$ \\
\hline Coronene & $0.05-0.3$ & $100 / 50$ & ED & 7.3 & 68) /69) \\
\hline Wet Catalase & 0.02 & 200 & ED & 28 & $71)$ \\
\hline $\begin{array}{c}\text { Uranyl-stained } \\
\text { catalase }\end{array}$ & 1.0 & $80 / 100$ & ED & 4.0 & $73) / 72)$ \\
\hline Para-terphenyl & 0.50 & 100 & ED & 5.7 & 65) \\
\hline $\mathrm{Ag}-\mathrm{TCNQ}$ & 0.3 & 100 & ED & 7.3 & $31), 32)$ \\
\hline $\mathrm{Cl}_{6}$-benzene & 0.50 & 100 & ED & 5.7 & 65) \\
\hline Phthalocyanine & 0.1 & $(60)$ & $\operatorname{ED}(5.7 \AA)$ & 13 & $57), 58)$ \\
\hline Zn-phthalocyanine & 1.0 & 100 & $\mathrm{ED}$ & 4.0 & 32) \\
\hline \multirow[t]{2}{*}{ Cu-Phthalocyanine } & $1-2$ & $(60)$ & $\operatorname{ED}(12.5 \AA)$ & 2.8 & $57), 58)$ \\
\hline & $1-2$ & 100 & ED & 2.8 & 67) \\
\hline $\mathrm{Cu}-\mathrm{Cl}_{16}-$ & 34 & 100 & $E D$ & 0.7 & 46), 66) \\
\hline phthalocyanine & $25-55$ & 100 & ED & 0.5 & $67)$ \\
\hline
\end{tabular}

cf. ED; electron diffraction, LM ; loss of mass, $C$; contrast, (at room temperature).

See References $((39),(57),(58),(74)$ and (75)) for other examples. 
more sensitive than ordinary electron-microscopic films for $500 \mathrm{KV}$ electrons ( see Chapter 1), were used for recording the electron diffraction patterns, and developed with the D-19 developer. The electron beam current applied to the specimen was measured with a $\mu \mu$-ammeter connected to a Faraday's cage inserted between the projector lens and the fluorescent screen.

The lattice spacings corresponding to (020) and (110) reflections of the $\alpha$-form are getting closer, starting at the dose of about 0.3 Coulombs $/ \mathrm{cm}^{2}$ ( this value may be defined as "critical dose"), and finally these two reflections merge into each other. At the dose of about 0.5 Coulombs/cm ("total end point dose"), amorphous halo rings start replacing crystalline reflections. In the case of the $\beta$-form, the lattice spacing of (40.0) reflection expands a little by irradiation, and halo rings appear at the dose of about 0.4 Coulombs $/ \mathrm{cm}^{2}$ ( this value corresponds to "critical dose" and also "total end point dose"). The polymer molecules which have benzene rings in their backbone chains seem to be somewhat more resistant against electron irradiation. As for PPX $\left(+\mathrm{CH}_{2}-\mathrm{CH}_{2}{ }^{-} \mathrm{n}\right)$ crystals, the total end point dose (TEPD) is about 10 times larger than that of polyethylene crystals. The TEPD's, namely, electron doses necessary for complete damage of various organic crystals, are summarized in Table 3-1, together with the measuring method and the estimated limit of detectability, for comparison with the PPX crystals. The doses in Table 3-1 are mostly 
measured for 100KV electrons.

The limit of detectability, $d(\AA)$ ( that is, the attainable 39,40

resolution, is given by the modified Rose equation,

$$
\mathrm{d}=1 / 5 \mathrm{C} \sqrt{\mathrm{EQ}},
$$

where $C$ is the contrast, which is defined as the difference in intensity between two image points separated by a distance of d, divided by the average intensity of the two points; $f$ is the net utilization factor, which takes into account the collection efficiency of any specified imaging mode, the quantum efficiency of the detector, and the fraction of the TEPD which is actually used to produce an image; $Q$ is the TEPD on specimen, in units of Coulombs $/ \mathrm{cm}^{2}$. All the $d$ values in Table $3-1$ are calculated using $f=0.25, C=0.1$. The $d$-value for each material is considerably consistent with the highest resolution of the micrographs obtained so far under ordinary conditions. The TEPD value of another accelerating voltage can be estimated from the dose-voltage relationship. The inelastic cross-section or the absorption is proportional to $(m \lambda)^{2}$, that is, $1 / \beta^{2}$, where $m$ is the mass of an accelerated electron, $\lambda$ the electron wavelength, and $\beta$ the ratio of electron velocity to light velocity, respectively. Therefore the TEPD seems to be proportional to $\beta^{2}$. In the case of polymer specimens, however, it was reported that the TEPD is proportional to $B^{3}$ rather than $\beta^{2}$. In either case, the use of higher accelerating voltage of electrons may be effective to reduce the radiation damage. 
for taking high resolution electron micrographs

As shown in Table $3-1$, the resolution expected from the TEPD of PPX crystals $\left(\sim 0.4\right.$ Coulombs $\left./ \mathrm{cm}^{2}\right)$ is about $6 \AA$ for ordinary exposure-levels, as calculated by Eq.(3-1). When we restrict the exposure to the levels that produce optical density between 0.1 and 0.3 , the micrographs with higher resolution than $6 \AA$ can be expected. The finite resolution of the recording medium, namely, the grain size of photographic films is more than $20 \mu \mathrm{m}$. Therefore, in order to obtain the micrograph having $2 \AA$ resolution ( which is needed to resolve individual polymer chains composing polymer single crystals), we must take photographs at a magnification (M) larger than $10^{5} \mathrm{x}$. According to the characteristic curve of the photographic film ( KODAK electron image film SO-163, see Fig.1-5 in Chapter 1), the exposure necessary to produce optical density, थ 0.3 at $M=10^{5} \mathrm{X}$ can be achieved within the TEPD of PPX crystals $\left(\sim 0.4\right.$ Coulombs $\left./ \mathrm{cm}^{2}\right)$. Since the optical density on the film is approximately proportional to the exposure ( the total number of electrons that fell on a unit area of the film, within our density 49,50 distribution on the image plane from the optical density distribution of a micrograph. Thus we adopted $M=10^{5} \mathrm{X}$ as the magnification in taking electron micrographs. 
No objective aperture was used in order to avoid astigmatism due to the deviation from the proper aperture position caused by frequent taking-in-and-off of the aperture. The specimen was mounted on a carbon- and gold-coated Triafol (cellulose acetobutyrate) microgrid on which very thin carbon supporting film ( which was produced by the indirect evaporation onto freshly cleaved surfaces of mica in the light of Johansen's procedure) ( 51 ad been deposited using a water surface ( see Figs.3-2(a) and ( $\left.a^{\prime}\right)$ ). Though no carbon supporting film should be used to avoid the noise, the single-layer crystals of the PPX $\beta$-form are bent even in fairly small holes of the microgrid without a supporting film, as we can see from irregular contours due to diffraction contrast ( Fig.3-2(b) ). Carbon- and gold-coating of Triafol microgrid serves to increase their mechanical strength as well as thermal and electric conductivities, as described in Chapter 1.

3-3 Procedures for taking high resolution electron micrographs and the method for judgement of the fidelity of an image

To reach the $2 \AA$ resolution, the astigmatism of the electron microscope objective lens must be compensated to rather small amount. We applied the accurate stigmating method described in Chapter 1, to correct the astigmatism at the region apart from the 


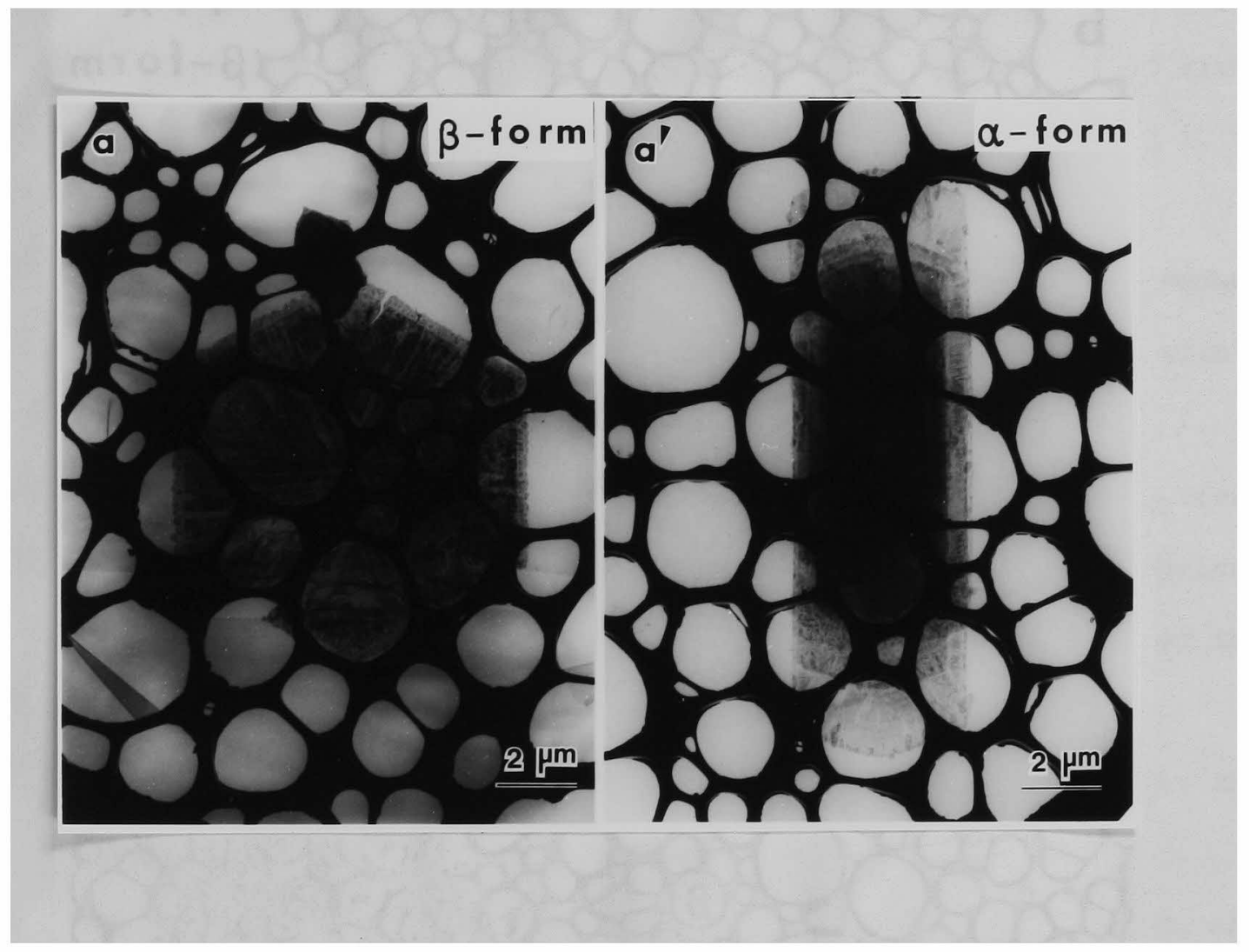

Fig.3-2 Specimens on a microgrid.

$\left(a, a^{\prime}\right)$ : The specimen is mounted on a $\mathrm{C}$ - and $\mathrm{Au}$-coated Triafol microgrid on which a very thin $C$ supportfilm has been deposited.

(b) : The specimen is mounted on a c-coated microgrid. Irregular contours due to bending are seen in a single-layer crystal (A) of the $B$-form, though regular radial stripes can be seen in a multilayer crystal (B). 


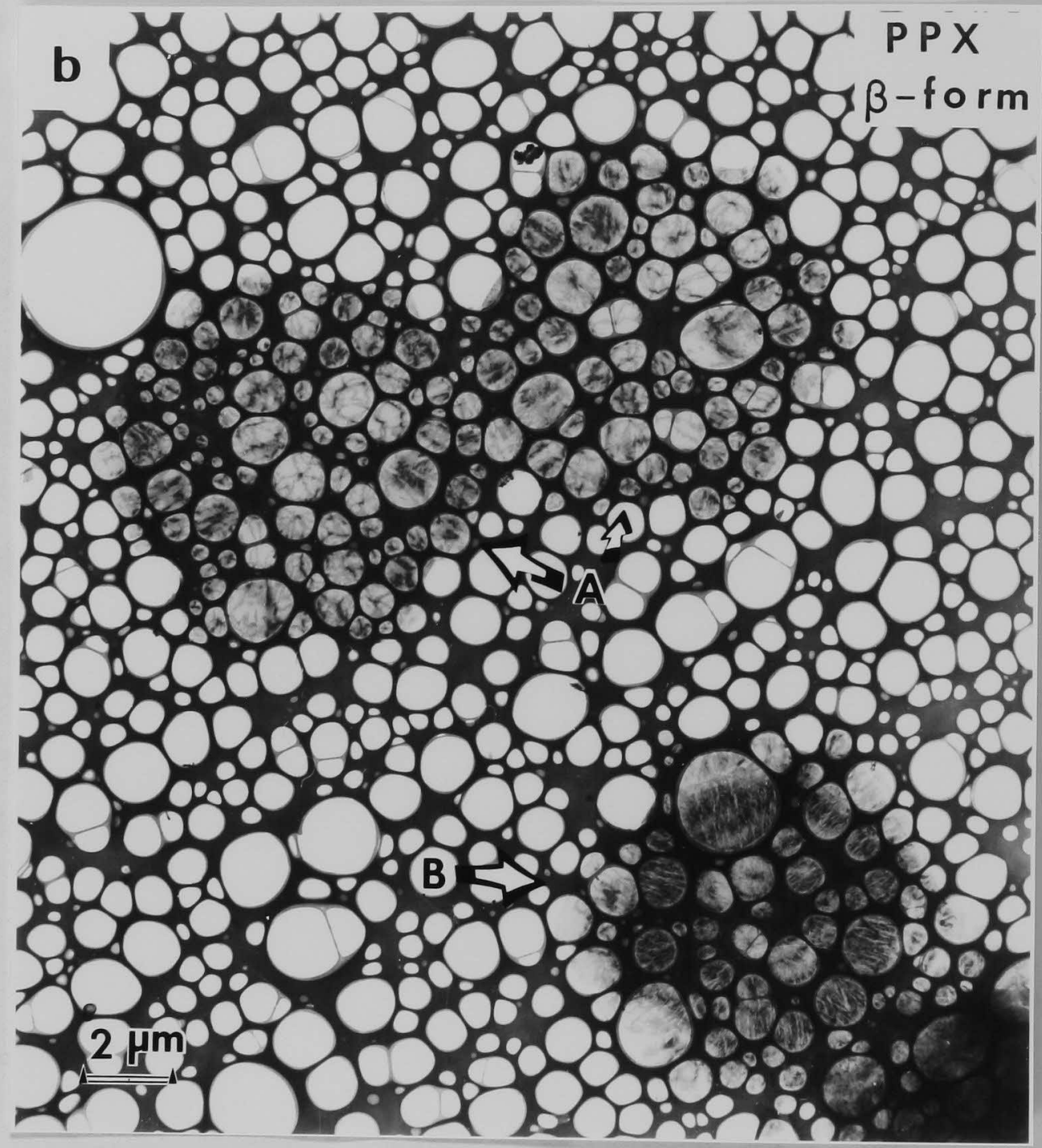

Fig.3-2 (continued) 
specimen to avoid the radiation damage.

Actual procedures of astigmatism correction are as follows:

1). We take 2 ' 3 through-focal micrographs of a thin amorphous object ( carbon support-film or amorphous specimen itself after electron irradiation ) at the assigned magnification ( $M=10^{5} \mathrm{X}$ ), if possible at an under-focus (weak lens).

2). These micrographs, having been developed, fixed, and washed with water, are dipped in ethyl alcohol and dried quickly with a drier.

3). These films are inserted at the object plane 0 in an optical diffraction apparatus (Fig.4-1 in Chapter 4), and the optical diffraction patterns are observed on the transmission screen at the plane F.

4). The difference in patterns due to through-focusing shows the defocus condition, and the most suitable micrograph is selected.

5). The selected micrograph is again inserted at the plane 0 , and the diffraction pattern is recorded on a Polaroid-Land film at the plane $\mathrm{F}$.

6). Based on this diffractogram, the correction values of the stigmator currents are estimated from Eqs.(1-29) and (1-30) in Chapter 1 .

After completion of the astigmatism correction at $M=10^{5} \mathrm{X}$, electron micrographs were taken according to the procedure shown 
below to minimize the radiation damage:

1). Search the proper specimen position in the diffraction mode with the electron beam spread widely with the second condenser lens (CL2) .

( The diffraction mode in JEM-500 allows to exhibit the specimen and its diffraction pattern simultaneously, when the beam is widely spread and no selected-area aperture is used. )

2). Shift the specimen position aside by $2 \sim 3 \mu \mathrm{m}$ and focus the beam on a new position with CL2.

3). After quick focusing in the imaging mode at $10^{5} \mathrm{X}$, the beam is spread again with CL2. Then shift the specimen back to the original position in diffraction mode.

4). Turn off the emission control and set the imaging mode to $10^{5} \mathrm{x}$. Then wait for $3 \sim 5$ minutes to avoid mechanical drift of the specimen stage and to regain the stability of lenses.

5). Turn on the emission control. Focus the electron beam quickly on the specimen with CL2 to the appropriate brightness determined in advance. A photographic film ( KODAK electron image film so-163) is exposed for $10 \sim 15$ seconds.

The photographic films were developed at $20^{\circ} \mathrm{C}$ for 3 minutes with KODAK developer D-19 diluted $1: 1$ and fixed with KODAK rapid fixer. The micrographs obtained were analyzed with optical transformation which will be described in the next chapter. If the 


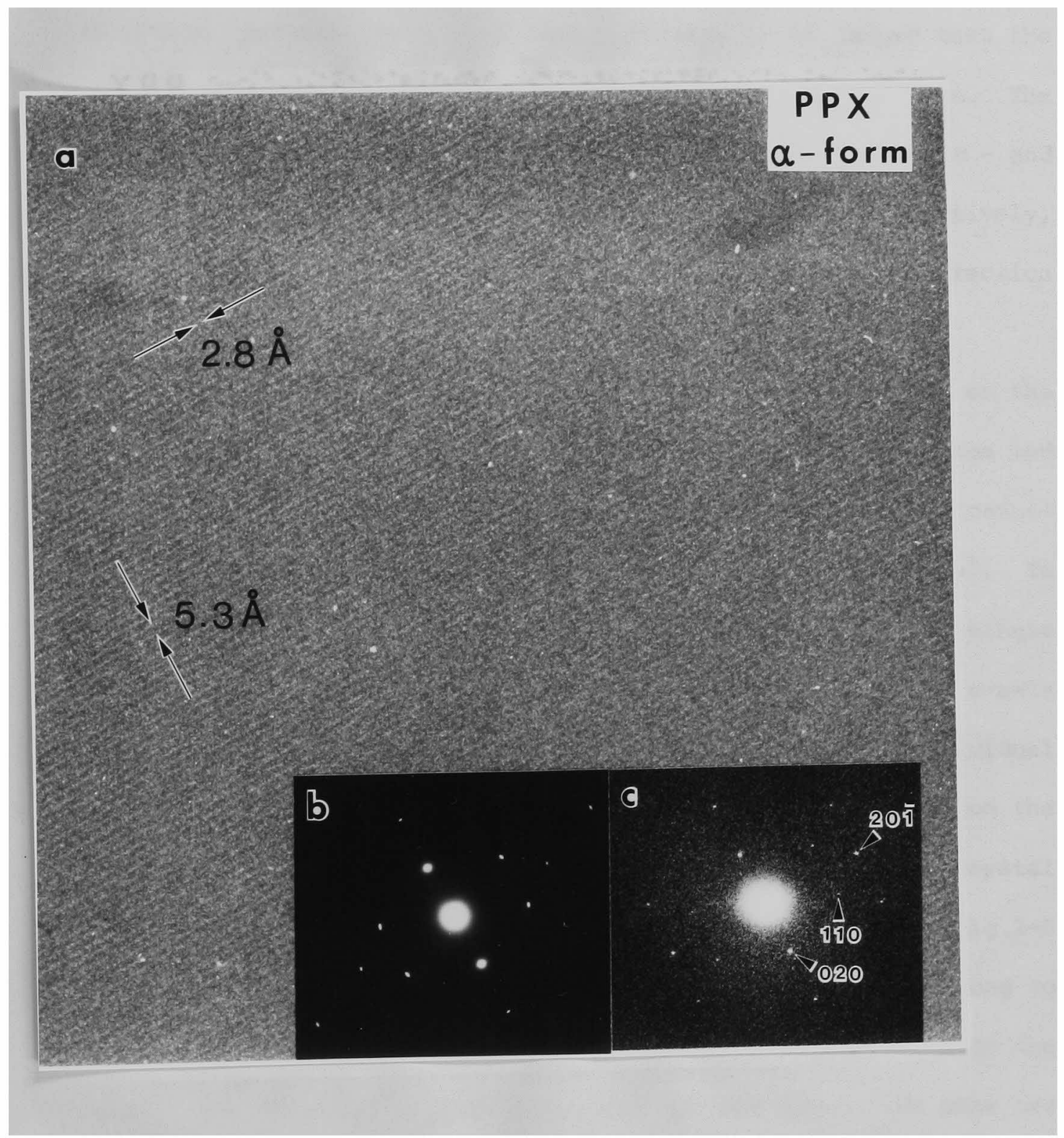

Fig.3-3 High resolution image of a PPX $\alpha$-form single crystal. (a) ; high resolution electron micrograph.

(b) ; $500 \mathrm{KV}$-electron diffraction pattern.

(c); optical diffractogram of (a). 


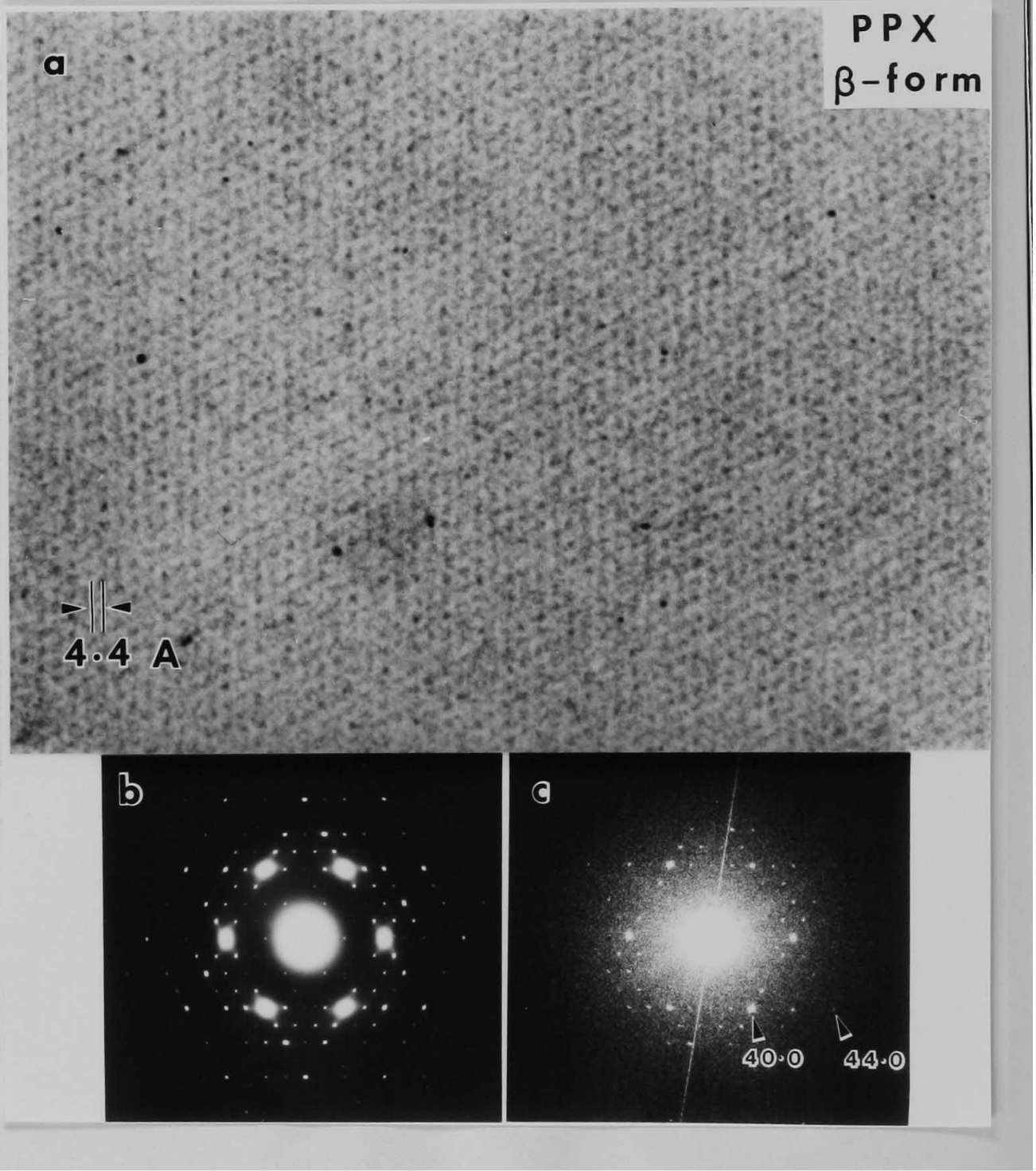

Fig.3-4 High resolution image of a PPX $\beta$-form single crystal.

(a) ; high resolution electron micrograph.

(b) ; $500 \mathrm{KV}$-electron diffraction pattern.

(c) ; optical diffractogram of (a). 
optical diffraction pattern from a micrograph resembles the electron diffraction pattern from the same specimen, it is judged that the micrograph reflects the true crystal structure of the specimen. The resulted high resolution electron micrographs of both PPX $\alpha$ - and $\beta$-form single crystals are shown in Figs.3-3 and 3-4, respectively, with their electron diffraction patterns and the optical diffraction patterns of the images.

As described in Chapter 2, the electron beam is incident on the single layer of an $\alpha$-form single crystal in the <102> direction and not parallel to the c-axis (molecular axis). Therefore we cannot resolve individual polymer chains of PPX in an $\alpha$-form crystal. In the case of the $\beta$-form, the electron beam is incident on the single crystal in the $<00.1\rangle$ direction and parallel to the c-axis (molecular axis). Therefore we are able to resolve the individual chains composing the $\beta$-form single crystal ( projection on the ab-plane, see Fig.3-4). As described in Chapter 2, the crystal structure of the PPX $\alpha$-form has already been analyzed ( see Fig.2-6 and Table 2-1 ). The PPX $\beta$-form crystal is proposed to belong to the hexagonal system and that the molecules are all parallel to the 54

c-axis. The detailed crystal structure is not known. We make use of this micrograph as a starting point of the crystal structure analysis. A similarity between the electron diffraction pattern (Fig.3-4(b)) of the $B$-form single crystal and the optical diffractogram (Fig.3-4(C)) of its high resolution micrograph 
(Fig.3-4(a)) shows that the micrograph sufficiently reflects the crystal structure of the $\beta$-form. However, because of the low $\mathrm{S} / \mathrm{N}$ ratio due to the photographic graininess the details expected from the diffractogram ( it shows up to (44.0) reflection) are not sufficient. The next chapter deals with an optical filtering method to improve the $\mathrm{S} / \mathrm{N}$ ratio of the micrograph. 
References

1) S.Iijima, J.Appl.Phys., 42, 5891 (1971).

2) S.Iijima \& J.G.Allpress, Acta Cryst., A-30, 22 (1974).

3) S.Iijima \& J.G.Allpress, ibid., A-30, 29(1974).

4) H.Hashimoto, A.Kumao, H.Endoh, H.-U.Nissen, A.Ono \& E.Watanabe, Proc.EMAG-75 Conf., Bristol, 245 (1975).

5) K.Kobayashi, ibid., 251 (1975).

6) S.Horiuchi, I.Kawada, M.Nakano-onoda, K.Kato, Y.Matsui, F.Nagata \& M.Nakahira, Acta Cryst., A-32, 558(1976).

7) S.Horiuchi, Y.Matsui \& Y.Bando, Japan.J.Appl.Phys., 15, $2483(1976)$.

8) N.Uyeda, Y.Fujiyoshi \& K.Ishizuka, Proc.US-Japan Seminar HVEM, Honolulu, (1976).

9) Y.Fujiyoshi, K.Ishizuka \& N.Uyeda, J.Electron Microsc., 26, 47 (1977).

LO) H.Hashimoto, H.Endoh, T.Tanji, A.Ono \& E.Watanabe, J.Phys.Soc.Japan, 42, 1073 (1977) .

L1) J.Desseaux, A.Renault \& A.Bourret, Phil.Mag., 35, 357(1977).

12) S.Horiuchi, T.Kikuchi \& M.Goto, Acta Cryst., A-33, 701 (1977).

13) K.Yagi \& J.M.Cowley, ibid., A-34, 625 (1978).

14) K.Izui, S.Furuno \& H.Otsu, J.Electron Microsc., 26, 129 (1977).

15) A.V.Crewe, J.Walland \& J.P.Langmore, Science, 168,1338 (1971).

16) H.Hashimoto, A.Kumao, K.Hino, H.Yotsumoto \& A.Ono, Japan.J.Appl .Phys . , 10, 1115 (1971) .

17) H.Formanek, M.Muller, M.H.Hahn \& T.Koller, Naturwissenschaften, 58, 339 (1971).

18) R.M.Henkelman \& F.P.Ottensmeyer, Proc.Nat.Acad.Sci.USA, 68, 3000 (1971).

19) E.B.Prestridge \& D.J.C.Yates, Nature, 234, 345 (1971).

20) A.V.Crewe, Proc.5-th Euro.Congr.EM, Manchester, 640 (1972).

21) J.R.Parsons, H.M.Johnson, C.W.Hoelke \& R.R.Hosbons, ibid., 646 (1972).

22) D.Dorignac, Proc.8-th Int.Congr.EM, Canberra, vol.1, 270 (1974)

23) H.Hashimoto, A.Kumao, K.Hino, H.Endoh, H.Yotsumoto \& A.Ono, J.Electron Microsc., 22, $123(1973)$.

24) S.Iijima, Optik, 48, 193(1977).

25) R.F.Whiting \& F.P.Ottensmeyer, J.Mol.Biol., 67, 173(1972).

26) F.P.Ottensmeyer, E.E.Schmidt \& A.J.Olbrecht, Science, 179 , 175 (1973).

27) N.Uyeda, T.Kobayashi, E.Suito, Y.Harada \& M.Watanabe, J.Appl.Phys., 43, 5181 (1972).

28) Y.Murata, J.R.Freyer \& T.Baird, J.Micrsc., 108, 261 (1976).

29) J.R.Fryer, Acta Cryst. , A-34, 603(1978).

30) J.R.Fryer, ibid., A-35, 327 (1979).

31) N.Uyeda, T.Kobayashi, K.Ishizuka \& Y.Fujiyoshi, Nature, 85, 95 (1980). 
32) Y.Fujiyoshi, T.Kobayashi, K.Ishizuka, N.Uyeda, Y.Ishida \& Y.Harada, Ultramicrosc., 5, 459 (1980).

33) G.A.Bassett \& A.Keller ; cited by A.Keller, Kolloid-Z, 231, 386 (1969).

34) M.G.Dobb, A.Hindeleh, D.J.Johnson \& B.P.Saville, Nature, 253, 189 (1975).

35) S.C.Bennett, M.Dobb, D.J.Johnson, R.Murray \& B.P.Saville, Proc.EMAG75, Bristol, 329 (1976).

36) M.G.Dobb, D.J.Johnson \& B.P.Saville, J.Polymer Sci.:Polymer Symp., 58, 237 (1977).

37) K.Kobayashi, E.Suito, N.Uyeda, M.Watanabe, T.Yanaka, T.Etoh, H.Watanabe \& M.Moriguchi, Proc.8-th Int.Congr.EM, Canberra, vol.1, 30 (1974).

38) Y.Fujiyoshi, Denken(Electron-Microsc.), 15, 72 (1980).

39) R.M.Glaeser, Proc.3-rd Int.Conf.HVEM, Oxford, 370 (1974).

40) I.A.M.Kuo \& R.M.Glaeser, Ultramicrosc., 1, 53 (1975).

41) H.Yoshioka, J.Phys.Soc.Japan, 12, 618(1957).

42) K.Kobayashi \& M.O'hara, Proc.6-th Int.Congr.EM, Kyoto, vol.1, $579(1966)$.

43) K.Kobayashi \& K.Sakaoku, Bull.Inst.Chem.Res., Kyoto Univ., 42,473 (1964).

44) K.Kobayashi \& K.Sakaoku, Labo.Invest., 14, 1097 (1965).

45) R.M.Glaeser, T.F.Budinger, P.M.Aebersold \& G.Thomas, Proc.7-th Int.Congr.EM, Grenoble, vol.1, 463(1970).

46) N.Uyeda, T.Kobayashi, M.O'hara, M.Watanabe, T.Taoka \& Y.Harada, Proc.5-th Euro.Congr.EM, Manchester, 566 (1972)

47) M.J.Richardsonn \& K.Thomas, ibid., 562 (1972).

48) G.C.Farnell \& R.B.Flint, J.Microsc., 97, 271 (1973).

49) R.C.Valentine, Labo.Invest., 14, 1334(1965).

50) R.C.Valentine, in "Advances in Optical and Electron Microscopy", R.Barer \& V.E.Cosslett Ed., vol.1, Academic-Press, $180(1966)$.

51) B.V.Johansen, Micron, $\underline{5}, 209$ (1974).

52) O.L.Krivanek, S.Isoda \& K.Kobayashi, J.Microsc., 111, 279 (1977).

53) R.Iwamoto \& B.Wunderlich, J.Polymer Sci.-Phys., 11, 2403(1973)

54) W.D.Niegisch, J.Appl.Phys., 37, 4041(1966).

55) L.Reimer, in Ref.(74), Chapter 3, (1975).

56) M.S.Isaacson, in Ref.(74), Chapter 14, (1975).

57) L.Reimer, Labo.Invest., 14, 1082 (1965).

58) L.Reimer, "Electronenmikroskopische Untersuchungs- und Praparationsmethoden", Springer-Verlag, Chapter 9, (1967).

59) G.Siegel, Proc.7-th Int.Congr.EM, Grenoble, vol.2, 221 (1970).

60) G.Ungar, D.T.Grubb \& A.Keller, Polymer, $21,1284(1980)$.

61) D.T.Grubb \& G.W.Groves, Phil.Mag., 24, 815(1971).

62) D.T.Grubb, A.Keller \& G.W.Groves, J.Materials Sci., ㄱ, 131 (1972). 
63) G.F.Bahr, F.B.Johnson \& E.Zeitler, Labo.Invest., 14, 1115 (1965).

64) M.G.Dobb \& R.Murray, J.Microsc., 101, 299 (1974).

65) G.M.Parkinson, M.J.Goringe, W.Jones, W.Rees, J.M.Thomas \& J.O.Williams, Proc.EMAG75, Bristol, 315(1976).

66) Y.Harada, T.Taoka, M.Watanabe, M.O'hara, T.Kobayashi \& N.Uyeda, Pro.30-th Annual Meeting, EMSA, Los Angeles, 686(1972).

67) W.R.K.Clark, J.N.Chapman, A.M.Macleod \& R.P.Ferrier, Ultramicrosc., $\underline{5}, 195$ (1980).

68) W.J.Claffey \& D.F.Parsons, Phil.Mag., 25, 637(1972).

69) S.M.Salih \& V.E.Cosslett, Phil.Mag., $\underline{30}, 225$ (1974).

70) R.W.Ditchfield, D.T.Grubb \& M.J.Whelan, Phil.Mag., 27, 1267 (1973).

71) V.R.Matrcardi, R.C.Moretz \& D.F.Parsons, Science, 177, 268 (1972).

72) R.M.Glaeser \& L.W.Hobbs, J.Microsc., 103, 209 (1975).

73) R.M.Glaeser, J.Ultrast.Res., 36, 466 (1971).

74) B.M.Siegel \& D.R.Beaman ed., "Physical Aspects of Electron Microscopy and Microbeam Analysis", John Wiley \& Sons, Chapter 12-15, (1975).

75) M.S.Isaacson, in "Principles and Techniques of Electron Microscopy", vol.7, ed. by M.A.Hayat, Van Nostrand Reinhold, 1 (1977). 
Chapter 4 : Image processing for electron microscopy

It is expected to improve the resolution of an electron microscope (EM) up to the theoretical resolving limit (see Chapter 1) for the direct observation of molecular and atomic images. This expectation has never been realized because of aberrations ( in particular, the spherical and chromatic aberrations) of the objective lens in EM and the photographic graininess in electron-microscopic films ( e.g., granularity of photographic emulsion, the quantum noise of electron, etc.). In 1974, Kobayashi et al. constructed a high resolution EM (JEM-500; its accelerating voltage is $500 \mathrm{KV}$ ) with a liquid helium specimen stage. With the small spherical aberration (spherical aberration coefficient; $\left.\mathrm{C}_{\mathrm{s}}=1.06 \mathrm{~mm}\right)^{7}$, this $\mathrm{EM}$ is designed to enable one to observe molecular or atomic images directly. The degradation of photographed images 
is mainly caused by the graininess. Though proposed various processing techniques including holographic filtering based on Gabor's holography, the degradation due to graininess should be eliminated by means of image processing prior to the correction of the effect of speherical aberration or defocus in order to obtain high resolution images.

The picture processing techniques have much progressed to meet the requirements from the space research, especially from the remote 15-17

sensing. In these techniques, pictures are replaced into the mathematical images, and the picture processing techniques such as the geometric correction, the noise removal, the high frequency nattenuation correction and so on are applied. These techniques have been applied to a posteriori image processing of electron micrographs, and are now indispensable for the high resolving 10,12

electron microscopy. Thus a computer image processing system was constructed as a part of our study on high resolution electron microscopy. Preliminary results were obtained by spatial frequency filtering and linear integration methods. A usual optical processing system was also constructed, and the results of image processing with this optical system were compared with those of the computer processing system. 
4-1 Construction of image processing systems

Image processing methods for electron microscopy fall into tw broad categories; (1) analogue and (2) digital processing. Althougl the apparatus for analogue processing ( usually a purely optica: method) is simple, this processing is poor in reproducibility an unsatisfactory for quantitative processing. The system of digital processing ( by means of a digital computer) is complicated ani requires more time to handle a large number of data. However this system shows good reproducibility and enables various kinds of processing with full accuracy, if the sampling method (especially, the sampling interval) is proper. ${ }^{18,20}$ Both analogue and digital processing methods were adopted and respective systems were constructed for image processing, especially for the removal of random noises caused by the photographic graininess in electron micrographs.

4-1-1 Analogue processing system for conventional optical filtering

The optical transform method, which was used for the first time

by Bragg in 1939 and developed by Taylor \& Lipson, has been used for the anticipation and/or the interpretation of $x-r a y$ or electron diffraction pattern, and also for the analysis of electron micrographs. ${ }^{32-40}$ Hosemann ${ }^{41}$ studied the paracrystalline structure in 
polymer crystals with this method. In the field of high resolution electron microscopy : this simple method has been a sine qua non for accurate stigmating of the objective lens ( see Chapter 1 ) or for the performance test of $\mathrm{EM}^{7}$. On the basis of the optical transform principle, Klug et al. ${ }^{43}$ established the so-called optical filtering method for image processing of electron micrographs and applied this to the analysis of large biopolymer structures. This method has progressed and been applied in the wide field of electron microscopy. ${ }^{44-56}$ From the processed images, reconstruction of 3-dimensional structures has been also attempted with a digital ${ }^{57-67}$ Recently special specimen preparation methods for image computer. Recently special specimen preparation methods for image processing in biological electron microscopy were also reported.

our apparatus was so constructed as to analyze electron micrographs by optical transforms and/or remove random noises in them by optical filtering. Figures 4-l(a) and 4-1(b) show the general view and the scheme of the apparatus, respectively. The light beam from the source s ( a He-Ne gas laser; the wavelength is $6328 \AA$ and out-put power $5 \mathrm{~mW}$ ) is focused at a point in the plane A with lens Lo (objective lens for an optical microscope; 10X or $20 \mathrm{x}$ ) and passes through a diaphragm ( $20 \mu \mathrm{m}$ in diameter; objective aperture for $\mathrm{EM}$ ). The passing point in the plane $\mathrm{A}$ is considered as an actual point source of light. The electron micrograph in the plane $O$ is illuminated by the light waves collimated with lens $\mathrm{L}_{c}$. The Fourier spectrum of the object is obtained on the back focal 
plane $F$ of lens $L_{d}$. The last lens $I_{i}$ transforms this spectrum to an image. A photograph of the spectrum or image can be taken with a camera set in the plane $F$ or in the image plane I. If a proper filter is set in the plane F, a processed image is formed in the plane I. The lenses $L_{c}, L_{d}$ and $L_{i}$ are $60 \mathrm{~mm}$ in diameter. This system is fixed on an iron timber ( $100 \mathrm{~mm} \times 50 \mathrm{~mm}$ in cross section and $3000 \mathrm{~mm}$ long) with magnetic stands, so the system can be rearranged easily in accordance with experimental requirements.

\section{4-1-2 Digital processing system}

An analogue processing method is sufficient to improve electron micrographic images up to the atomic order resolution, but offers little variety in the modes of image processing. On the contrary, digital processing has wider application, and various methods have $10-12,16,69-77,94$

been proposed. An apparatus for digital image processing was constructed. A general view of this apparatus and a schematic flow of data are shown in Figs.4-2(a) and 4-2(b). The optical density distribution of an electron micrograph is measured automatically with a Joyce-Loebl double beam microdensitometer. Its sample stage is driven by programmable stepping-motors in two independent $(x, y)$ directions and the basic interval is $5 \mu \mathrm{m}$ in each direction. Optical density in each image element, determined by a programed interval, is digitized with an analogue-to-digital converter within 


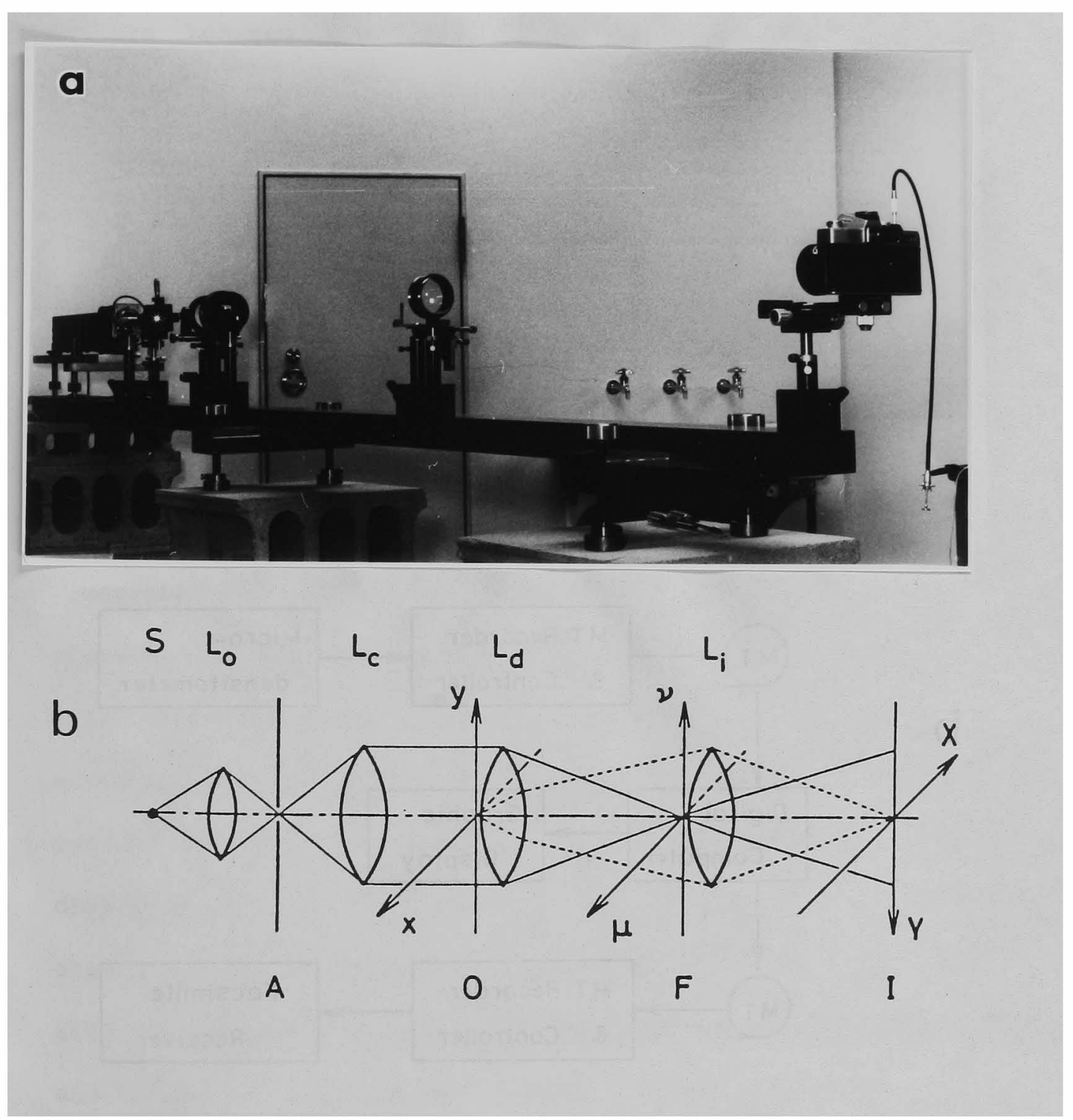

Fig.4-1 Apparatus for optical filtering.

(a) General view.

(b) Schematic arrangement.

$\mathrm{S}$; light source (a He-Ne gas laser), $\mathrm{L}_{\mathrm{O}}$; condenser lens, $\mathrm{L}_{\mathrm{C}}$; collimating lens, $\mathrm{L}_{\mathrm{d}}$; diffraction lens, $\mathrm{L}_{i}$; imaging lens, $A_{\text {; }}$ aperture, $\mathrm{O}$; object plane, $F$; back focal plane of lens $I_{d}$, $I_{;}$image plane. 

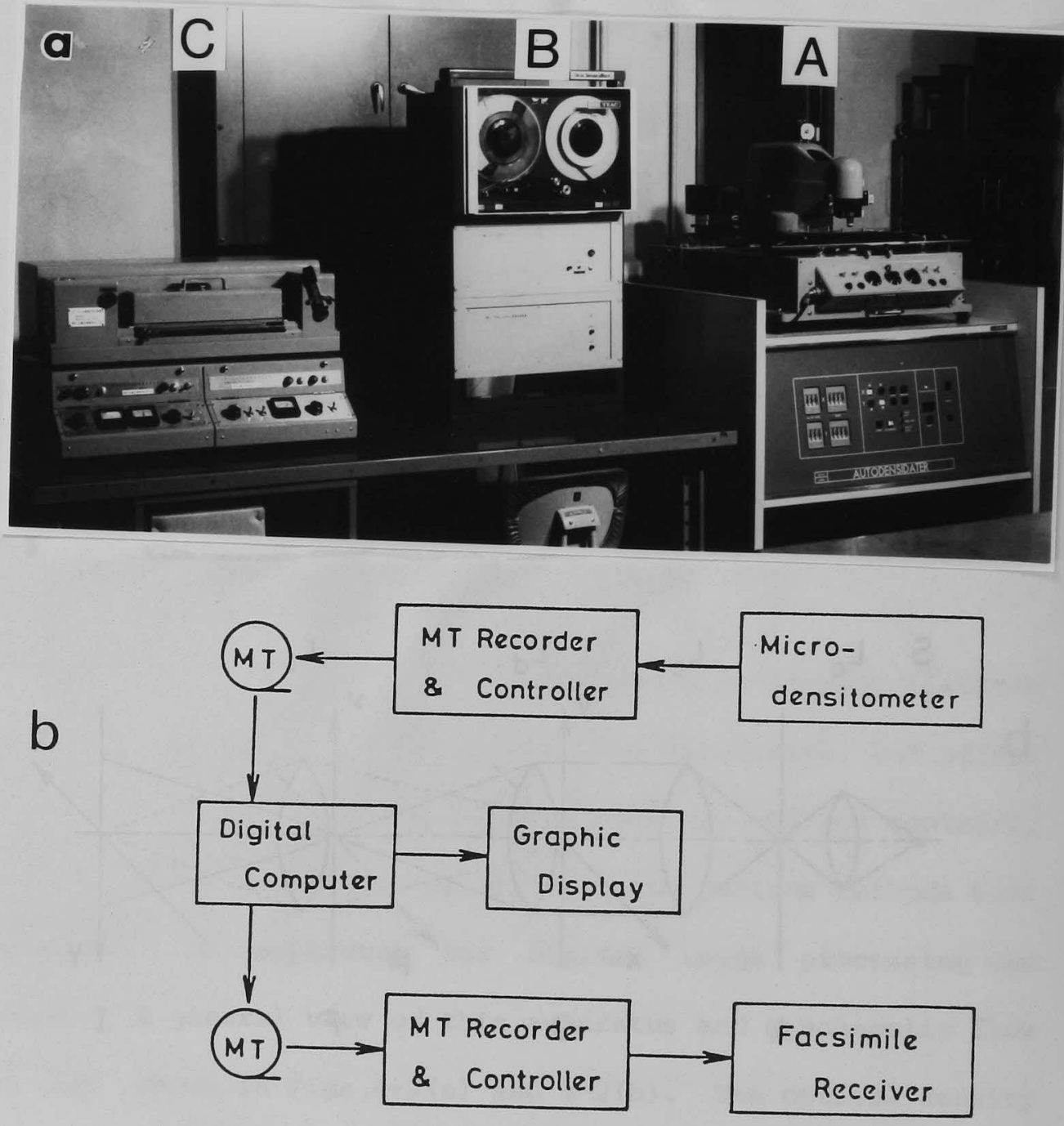

Fig.4-2 Apparatus for digital image processing.

(a) General view.

$A_{\text {; }}$ microdensitometer, $B_{\text {; }}$ magnetic tape recorder and its controller, $C_{i}$ facsimile receiver.

(b) Schematic flow of data. 
the microdensitometer. These digitized data consist of integral numbers between 0 and 999. With a magnetic tape (MT) recorder and its controller, a pack of 1024 digitized data in the $x$-direction (y fixed) can be recorded in increasing order of $y$ on MT for numerical processing by digital computer. The data processed by computer are again recorded on MT and converted to analogue data with a digital-to-analogue converter within MT controller. These analogue data are displayed directly on a photographic paper as a 2-dimensional analogue picture with a facsimile receiver. One picture element displayed with the facsimile receiver has a dimension of $0.196 \mathrm{~mm} \times 0.212 \mathrm{~mm}$, and the maximum number of picture elements is 970 in the $x$-direction and 1200 in the $y$-direction. Gray scale displayed with this apparatus is shown in Fig.4-3. Although 20 levels are illustrated in this figure, actual pictures consist of 256 tone-levels. It is confirmed that the image displayed through this system is practically identical to the original one, as shown in Fig.4-4. This facsimile receiver is also used to display the simulated through-focal EM images ( Chapter 5) and the result of $\mathrm{x}$-ray diffraction pattern simulation. 
Fig.4-3 Gray scale shows 20 discrete levels.

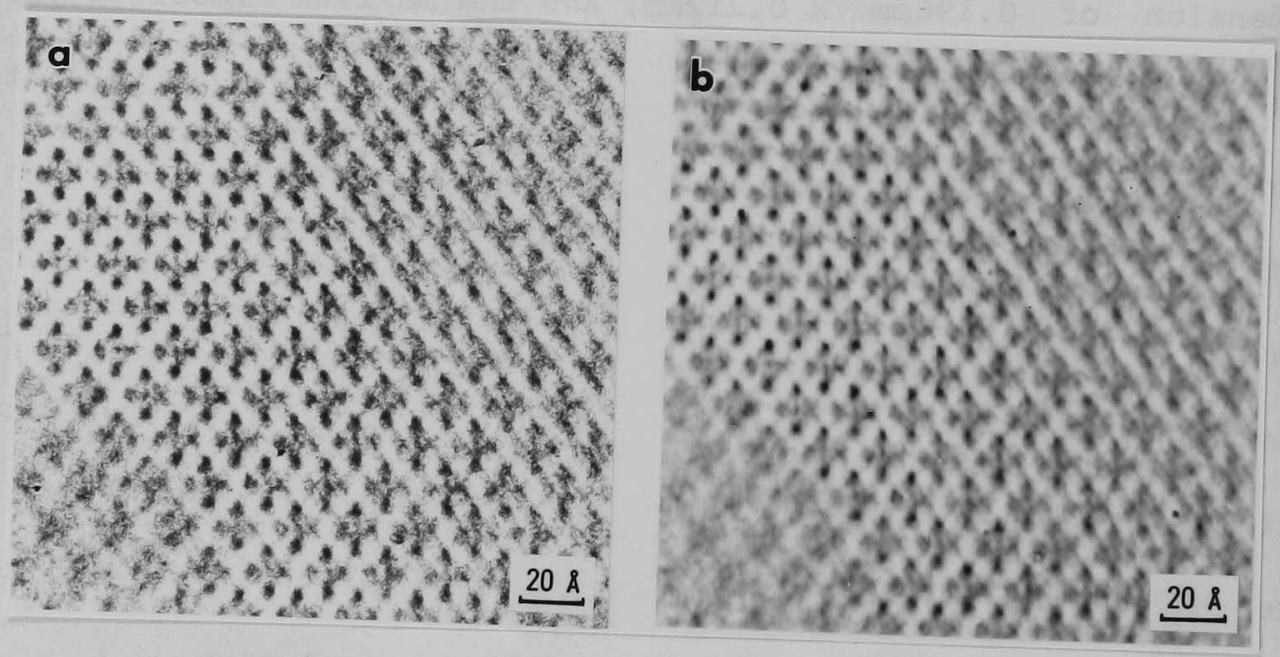
Fig.4-4 Molecular images of hexadecachloro- $\mathrm{Cu}-\mathrm{phthalocyanine}$
taken at 500kV.

(a) ; enlarged electron micrograph.

(b) : picture displayed with the facsimile receiver
without any processing. 


\section{4-2-1 General background}

An optical filtering system is shown in Fig. 4-1(b). Suppose that an electron micrograph with amplitude transmittance $g\left(x_{0}\right)$ is set in the object plane $O$ in front of the lens $L_{d}$ and illuminated with a collimated laser beam. Then, the amplitude distribution $G(u)$ in the back focal plane $F$ of lens $L_{d}$ can be expressed in terms of 2-dimensional Fourier transform as

$$
G(u)=\int g\left(x_{0}\right) \exp \left\{2 \pi i u x_{0}\right\} d x_{0},
$$

where the variables $u$ and $x_{0}$ are the spatial frequencies in Fourier space ( $p l a n e F)$ and the position coordinates in the object plane 0 , respectively, and a constant phase factor is neglected. If a camera is set and the intensity $|G(u)|^{2}$ is recorded at this place (plane F), we can obtain the spatial frequency distribution (viz., an optical diffraction pattern) of the object $g\left(x_{0}\right)$. After passing through the plane $\mathrm{F}$ the light waves form a real image on the plane I (i.e., the lens $L_{i}$ also produces the Fourier transform). If we take the coordinate axes in the image plane I in the opposite directions to those in the plane $O$ as shown in Fig.4-1(b) and we define a proper scale proportional to the magnification, then the amplitude distribution $\psi\left(x_{i}\right)$ in the plane $I$ is given by

$$
\psi\left(x_{i}\right)=\int G(u) \exp \left\{-2 \pi i x_{i} u\right\} d u,
$$

When we put a filter with amplitude transmittance $T(u)$ in the 
plane $F$, the modified amplitude distribution $\psi^{\prime}\left(x_{i}\right)$ on the imac plane $I$ is written by

$$
\begin{aligned}
\psi^{\prime}\left(x_{i}\right) & =\int G(u) T(u) \exp \left\{-2 \pi i x_{i} u\right\} d u \\
& =g\left(x_{i}\right) \star t\left(x_{i}\right),
\end{aligned}
$$

where $t\left(x_{i}\right)$ is the inverse Fourier transform of $T(u)$ and the denotes the convolution operation. The quantity $\left|\psi^{\prime}\left(x_{i}\right)\right|^{2}$ is the intensity distribution of a filtered image. This method is generally called a "spatial frequency filtering". The correction of the spherical aberration will be possible with a proper filter $T(u)^{74}$

If random noises caused by the photographic graininess are superimposed on a periodic pattern, then $G(u)$ consists of not only a set of discrete peaks due to periodicity, but also a continuous spectrum due to noises. If we insert a filter grating $T(u)$ in the plane $F$ which can let through the sharp peaks but block the rest of the spectrum, the periodic structure will be enhanced with respect to the noise.

There is another method to remove random noises, called a "linear integration", $1.8 .$, , the shifted superposition of a periodic picture. If the noises are regarded as random, the $\sqrt{\mathrm{N}}$ improvement ${ }^{16}$ in the $\mathrm{S} / \mathrm{N}$ ratio will be attained by this procedure, where $\mathrm{N}$ is the number of repeated superposition. These two methods are different in practice. In a special case, however, Hashimoto et

al. and Aebi et al. have shown that the optical image contrast

al. and Aebi et al. have shown that the optical image contrast 
formed by spatial frequency filtering using a filter grating is equivalent to the picture contrast by linear integration. In fact, processed images by two methods were almost in our preliminary experiments.

identical

In the next section we shall outline a theory of "image averaging", based on the mathematical treatment by Aebi et al. and a scattering theory from imperfect crystals by Cowley, in order to clarify the meaning of averaging.

4-2-2 Theoretical treatment of image averaging

The function form of an ideal image is

$$
g(x)=s(x)[f(x) \star 1(x)],
$$

where $I(x)$ is an infinite 2-dimensional periodic array of delta functions; $f(x)$ is a function which describes the motif, i.e., the structure of a unit cell; $s(x)$ is a shape function which defines the overall size of the image, and in our case (a circular area) $s(x)=1$ within this area or $s(x)=0$ otherwise. Using a capital letter to denote the Fourier transform of a real space function expressed by the corresponding small letter, the Fourier transform of Eq. (4-4) is written by

$$
G(u)=s(u) *[(1 / \sigma) L(u) F(u)] \text {, }
$$

where $\sigma$ is the area of a unit cell in the real space, $L(U)$ is an infinite 2-dimensional array of delta functions identical to the reciprocal lattice of $l(x)$, and $F(u)$ corresponds to "structure 
Factor" in X-ray or electron crystallography.

The basic form of the transform is an array of delta functions $L(u)$ modulated by $F(u)$. The convolution operation then replaces each delta function with the transform $s(u)$. In practical cases the overall size is so large that the region is very narrow where $s(u)$ has a significant value. Therefore $G(u)$ is large only in small regions around the lattice points of $L(u)$, and the optical diffraction intensity is given by

$$
|G(u)|^{2} \cong|S(u)|^{2} \nleftarrow\left\{\left(I / \sigma^{2}\right) L(u)|F(u)|^{2}\right\} \text {. }
$$

This equation denotes that the optical diffraction pattern consists of the discrete set of sharp peaks.

In practice the experimental data $g^{\prime}(x)$ will not have the ideal form just defined. We may, however, write the measured data as the sum of a noise component $n(x)$ and a true structure $f(x) \star I(x):$

$$
g^{\prime}(x)=s(x)[f(x) \star 1(x)+n(x)]
$$

Now we define $\left\langle g^{\prime}(x)\right\rangle$ as the average periodic pattern in $g^{\prime}(x)$ and $f^{\prime}(x)$ as the motif of $\left\langle g^{\prime}(x)\right\rangle$. $f^{\prime}(x)$ may be different from $f(x)$ due to the noise, then

$$
\begin{aligned}
g^{\prime}(x) & =\left\langle g^{\prime}(x)>+\Delta g(x)\right. \\
& =s(x)\left[f^{\prime}(x) \star I(x)\right]+\Delta g(x),
\end{aligned}
$$

where $\Delta g(x)$ represents the deviation from the average pattern. The real function $\Delta g(x)$ is essentially non-periodic and not always equivalent to $s(x) n(x)$ unless $n(x)$ takes an ideally random value 
Iround zero. The Fourier transform of Eq. (4-8) is written by

$$
\begin{aligned}
G^{\prime}(u) & =\left\langle G^{\prime}(u)\right\rangle+\Delta G(u) \\
& =S(u)+\left[F^{\prime}(u)(I / \sigma) L(u)\right]+\Delta G(u),
\end{aligned}
$$

and the diffraction intensity is

$$
\begin{aligned}
\left|G^{\prime}(u)\right|^{2}= & \left|\left\langle G^{\prime}(u)\right\rangle\right|^{2}+|\Delta G(u)|^{2} \\
& +\left\langle G^{\prime}(u)\right\rangle \cdot \Delta G^{*}(u)+\left\{\left\langle G^{\prime}(u)\right\rangle\right\}^{*} \cdot \Delta G(u),
\end{aligned}
$$

where * denotes a complex conjugate. With spatial averaging, the rourier transform of the third term of the right side of Eq.(4-10) is given by

$$
\begin{aligned}
\left\langle g^{\prime}(x)\right\rangle \star \Delta g(-x) & =\left\{s(x)\left[f^{\prime}(x) \star I(x)\right]\right\} \star \Delta g(-x) \\
& \cong f^{\prime}(x) \star\{[s(x) I(x)] \star \Delta g(-x)\} \\
& \cong 0 \quad .
\end{aligned}
$$

fere the convolution of $\Delta g(-x)$ with $s(x) l(x)$ represents the sum $\sum_{j} \Delta g\left(x_{j}-x\right)$, where $x_{j}$ is the lattice vector, namely, the position rector of the point at which a $\delta$-function locates. If the image :ontains fairly large number of unit cells, this sum is nearly zero :or all $x$. Thus the relation $(4-11)$ is proved. The same applies :o the convolution of the fourth term.

Hence,

$$
\begin{aligned}
&\left.\left|G^{\prime}(u)\right|^{2} \cong\left|<G^{\prime}(u)\right\rangle\right|^{2}+|\Delta G(u)|^{2} \\
&=\left|S(u) \star\left[F^{\prime}(u)(1 / \sigma) L(u)\right]\right|^{2}+|\Delta G(u)|^{2} \\
& \cong|S(u)|^{2} \star\left\{\left|F^{\prime}(u)\right|^{2}\left(1 / \sigma^{2}\right) L(u)\right\}+|\Delta G(u)|^{2} .
\end{aligned}
$$

$|\Delta G(U)|^{2}$ in Eq.(4-12) represents a continuous distribution of cattering power between the reciprocal lattice points; i.e., 
diffuse scattering in optical diffraction pattern. Of course the first term of Eq.(4-12) gives sharp peaks due to $\left\langle\mathrm{G}^{\prime}(\mathrm{u})\right\rangle$.

A suitable filtering procedure should be found to transmit an optimal amount of information on $f(x)$ and eliminate as much noise as possible. In practice, the motif may be deformed owing to the noise, then only the reduction of $\Delta g(x)$ is possible and we can obtain only $f^{\prime}(x)$ in principle.

We construct a filter grating $T(u)$ as such

$$
T(u)=A(u)[W(u) \star L(u)] \text {, }
$$

where $W(u)$ is the filter hole function (a circle); and $A(u)$ is the band limiting function ( viz., aperture function) relating to the overall shape of the filter. Fourier transforms which we deal with are practically zero outside the filter. Then

$$
A(u)=1 \text {, for all } u \text {. }
$$

With this approximation $T(u)$ can be rewritten as

$$
T(u)=w(u) \star L(u) \quad \text {. }
$$

When we insert this filter at the plane $F$ in Fig.4-1(b), the modified image-amplitude $\psi^{\prime}(x)$ in the plane $I$ is given from Eq. (4-3) as

$$
\begin{aligned}
\psi^{\prime}(x) & =t(x) \star g^{\prime}(x) \\
& =[w(x) \sigma I(x)] \star g^{\prime}(x) \\
& =[w(x) \sigma I(x)] \star\left\{\left\langle g^{\prime}(x)\right\rangle+\Delta g(x)\right\} .
\end{aligned}
$$

Consequently, $\psi^{\prime}(x)$ is composed of superposed images with a shift $-x_{j}$, each contribution being weighted by the factor $w\left(x_{j}\right)$, where 
$x_{j}$ is the lattice vector. In the limiting case where $w(u)=\delta(u)$ and $w(x)=1$, the filtering is identical to the Markham superposition, viz., linear integration. This fact was also pointed out by Hashimoto et al. Here

$$
\begin{aligned}
\psi^{\prime}(x) & =\sigma I(x) \star\left\{\left\langle g^{\prime}(x)\right\rangle+\Delta g(x)\right\} \\
& =\sigma I(x) \star\left\langle g^{\prime}(x)\right\rangle \\
& =\sigma I(x) \star\left\{s(x)\left[f^{\prime}(x) \star I(x)\right]\right\},
\end{aligned}
$$

because $\Delta g(x) \star I(x)=0$ as described before. Equation (4-17) shows the convolution of the function $\left\langle g^{\prime}(x)\right\rangle$ defined in a finite area with the infinite 2-dimensional array of delta functions $1(x)$. For the similar reason, we realize from Eqs. (4-16) and (4-17) that the- processed image more spreads out of the original area of $s(x)$, with the narrower hole size $W(u)$ of the filter.

The effective averaging is thus determined by the spatial extent of $w(u)^{85}$ or the region where $w(x)$ has a significant value. (The smaller a spatial extent of $W(u)$, the larger such a region of $w(x)$. ) Fraser \& Millward discussed the effects of hole size $W(u)$, and estimated the optimum filter-hole size. If both the periodic and the large scale irregular structures are contained in the original image to be processed, we should carry out the optical filtering using a filter grating $T(u)$ which has pinholes of rather large size as shown in section 4-4. 
4-3 Optical filtering of the high resolution electron micrograph of the PPX $B$-form single crystal

The photographic graininess yields much noise in the high resolution micrograph of the PPX $\beta$-form single crystal to such an extent to obscure the details expected from its diffractogram ( it shows up to (44.0) reflection. See Fig.3-4(c) or 4-6(b).). We performed optical filtering with a hexagonal filter grating (lattice constant $1 \mathrm{~mm}$ ) having very small holes ( $100 \mu \mathrm{m}$ in diameter). This filter is shown in Fig.4-5. In our experiment, only the holes which correspond to the sharp peaks in the diffractogram were used and the others were clogged. This filter was properly inserted in the plane $F$ in Fig.4-1(b) and accentuated the periodical structure. In this case, the hole size of the filter is so small that we can expect the effective averaging, as described in previous section. Processed images were recorded on MINICOPY FILM (Fuji Photo Film Co. Ltd.) to avoid the quality degradation due to the granularity, and the film was developed at $20^{\circ} \mathrm{C}$ for 6 minutes with KONIDOL FINE (KONISHIROKU Photo Ind. Co. Ltd.) to reduce the high contrast of this film. Both the processed image and the model structure ( which was made on the basis of the result of structure analysis by electron diffraction. See Chapter 5.) are shown in Fig.4-6 (c\& $\left.\mathrm{C}^{\prime}\right)$. Each black spot in this photograph corresponds to a projected molecule along the polymer chain. This processed image shows mutual 


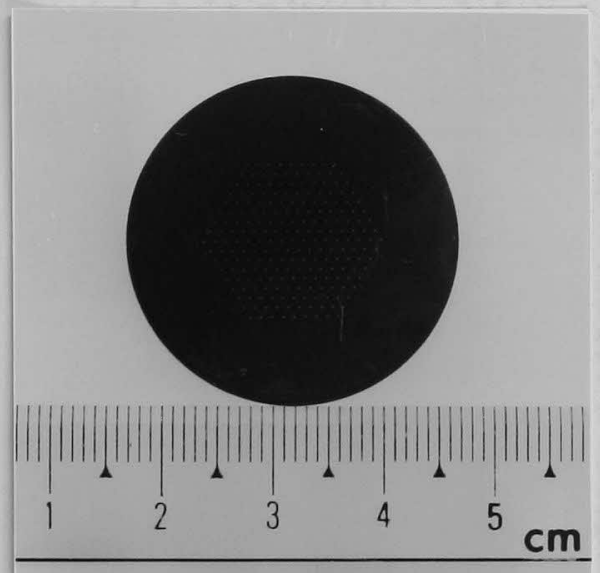

a

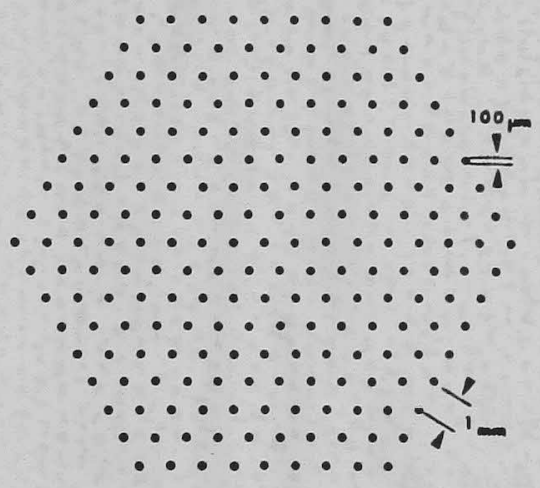

b

Fig.4-5 Filter grating for the PPX $\beta$-form in optical filtering.

(a) ; general view (stainless steel plate; $30 \mathrm{~mm}$ in diameter and $0.2 \mathrm{~mm}$ thick).

(b) ; schematic arrangement of pinholes in (a). 


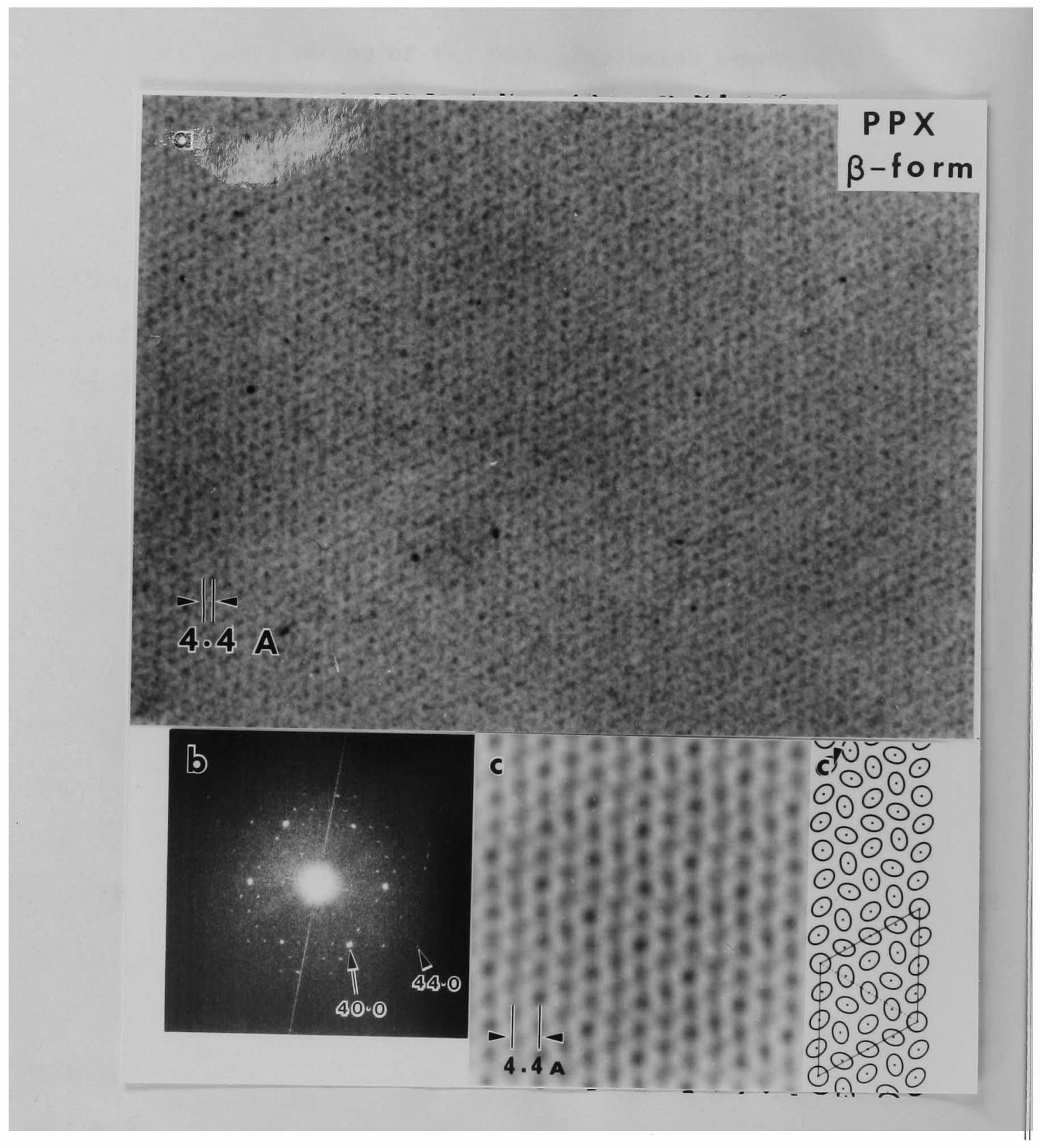

Fig.4-6 High resolution image of a PPX $\beta$-form single crystal.

(a) Original electron micrograph (ab-plane projection).

(b) Optical diffractogram of (a).

(c) Optically filtered image of (a) using the filter grating shown in Fig.4-5. (c') shows the mode. structure (see Chapter 5). 
positions of the chains in a unit cell as projected on the ab-plane. The detailed crystal structure of the PPX $B$-form crystal has not yet been analyzed. The next chapter deals with the analysis of the crystal structure of the PPX $\beta$-form based on this processed image.

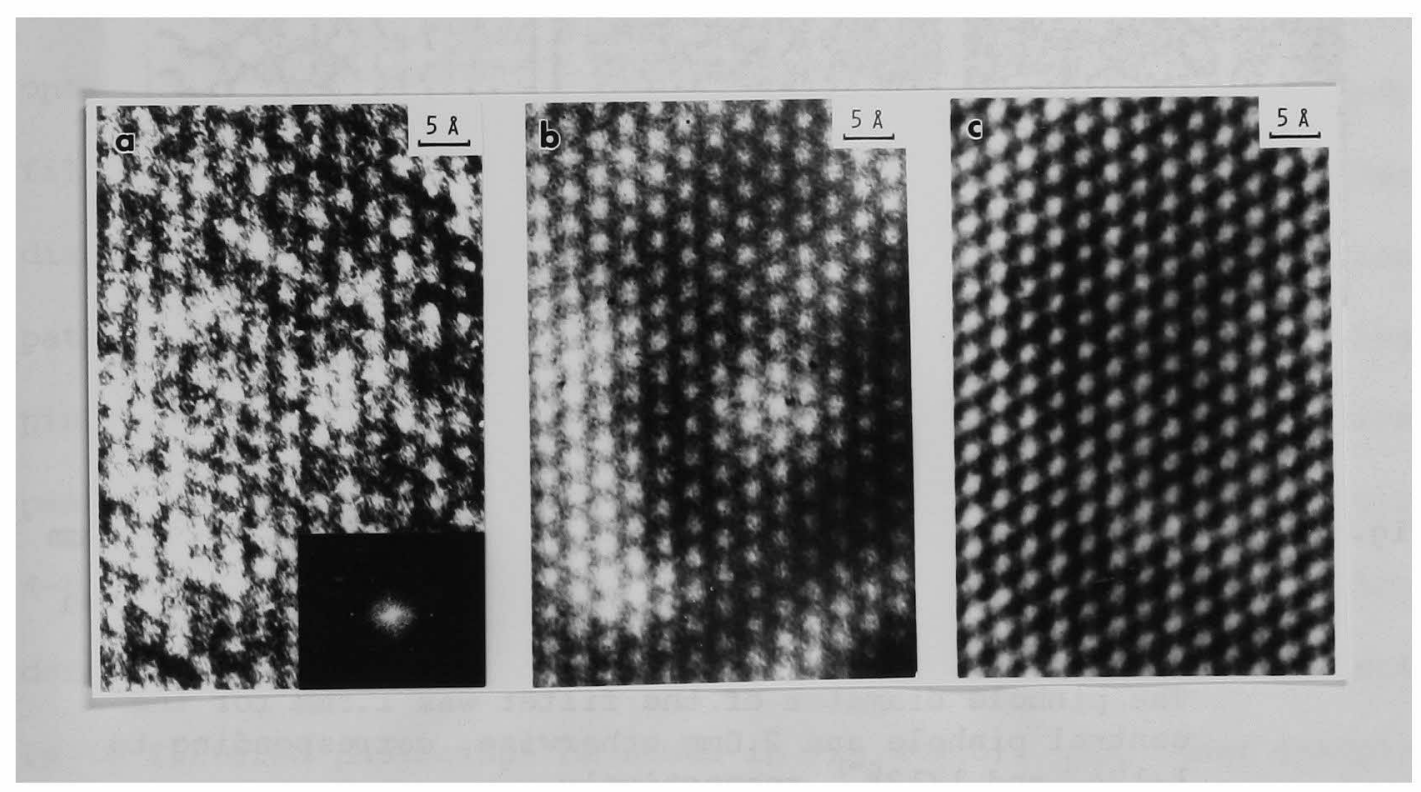

Fig.4-7 High resolution image of a thin crystalline film of cleaved molybdenite taken at $500 \mathrm{KV}$.

(a) $\mathrm{MOS}_{2}$ networks (projected on the ab-plane). The insertion shows the optical diffraction pattern of the image. The pinhole diamter of the filter was 2.0mm for the central pinhole and $1.1 \mathrm{~mm}$ for others, corresponding to $1 / 8 \AA^{-1}$ andl $/ 15 \AA^{-1}$, respectively.

(b) Optically filtered image of (a).

(c) Photographically liner-integrated image of (a). 

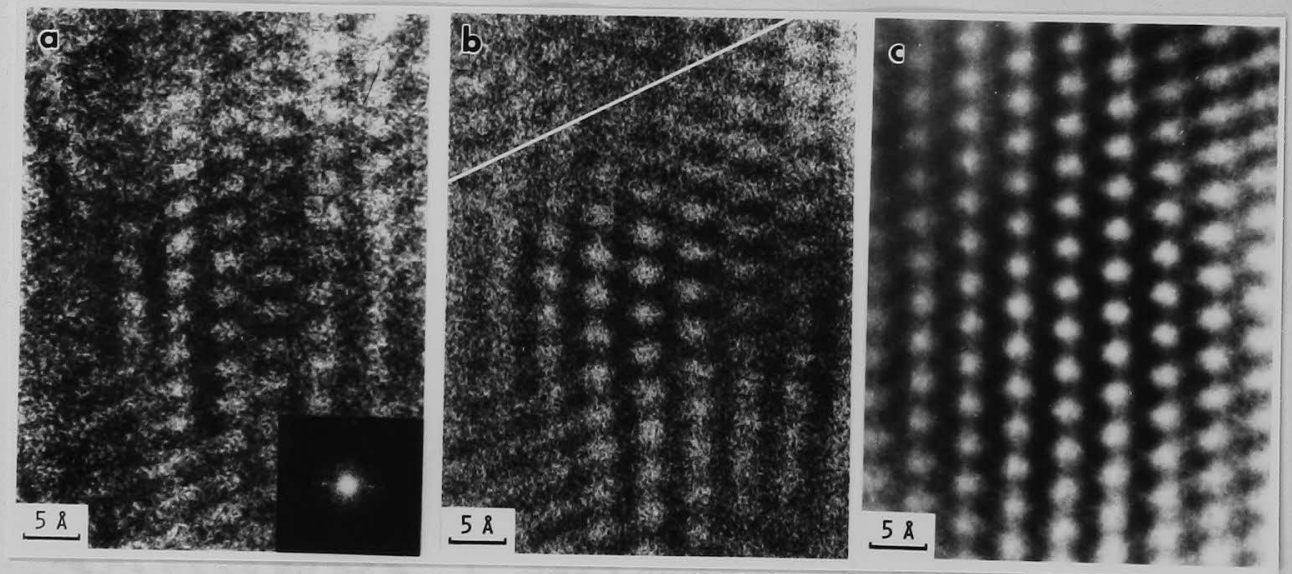

Fig.4-8 High resolution image of a fibrous crystallite of vanadium oxide (Type F-2), including lattice defects.

(a) $\mathrm{V}_{2} \mathrm{O}_{5}$ networks taken at $500 \mathrm{KV}$ (bc-plane projection) ${ }^{90}, 91$ The insertion shows the optical diffraction pattern. The pinhole diameter of the filter was $1.5 \mathrm{~mm}$ for the central pinhole and $2.0 \mathrm{~mm}$ otherwise, corresponding to $1 / 17 \AA^{-1}$ and $1 / 13 \AA^{-1}$, respectively.

(b) Optically filtered image of (a), which shows the halfperiod shifts of zigzag layer lines along the oblique line more clearly than (a).

(c) Photographically linear-integrated image of (a). 
The random noise removal in electron micrographs was performed by photographic linear integration ( viz., a multiple exposure technique combined with the periodic translation using a conventional enlarger). The linear integration method gave better results with respect to the $S / N$ ratio than optical filtering with a filter having rather large holes. Examples of these two processing methods are shown in Figs.4-7 and 4-8. The size of pinholes used in optical filtering is shown in the caption of each figure. A handy filter grating for optical filtering is formed by making holes directly in the Polaroid-Land film on which optical diffraction pattern of the micrograph is recorded. Since the filter has pinholes of finite size, the surrounding region of each diffraction peak as well as the peak itself is allowed to pass through. Figure 4-8(a) is the high resolution lattice image including a lattice defect, and optical filtering proves to reproduce the lattice defect in a filtered photograph as shown in Fig.4-8(b). The other example is seen in Fig.4-7(b) which corresponds to bright and dark areas of the original micrograph (Fig.4-7(a)). Thus the filtering method with a proper filter is more adequate to reduce random noises when the periodic/large-scale-irregular structures should be reproduced in processed images. The linear integration procedure is not adequate to the irregular structures ( see Fig.4-8). 
In PPX single crystals, lattice defects (edge dislocation) were resolved with JEM-500. Figure 4-9 shows the defect in the original lattice-image of the $\alpha$-form crystal and its optically processed image. Figure 4-10 reveals the edge dislocation in the $\beta$-form crystal and the processed image. In this case, we used the filter having larger holes than that used in Fig.4-6.

In both cases of Figs.4-9 and 4-10, the dislocations were barely found in enlarged photographs (Figs.4-9(a) and 4-10(a)). We photographically reduced the areas including dislocations in these photographs to use as object-micrographs for optical filtering, because we could not locate the dislocations in the original micrographs. In Fig.4-10, the object-micrograph was not large enough to accomodate many unit cells. 92.93 (This corresponds to the case where the overall size of the image to be processed is small, then $\mathrm{S}(\mathrm{u})$ in Eq. (4-5) will be a rather broad function.) It also lost the details of the original because of the several photographical steps. Figure 4-10(b) thus reveals only six (40.0) reflections. It was reported that optical diffraction patterns of the model grating representing edge dislocation showed the reflections which have the shape of a figure of ' 8 '. In our case, each diffraction spot was very broad, and such a shape of a diffraction spot was not found. 


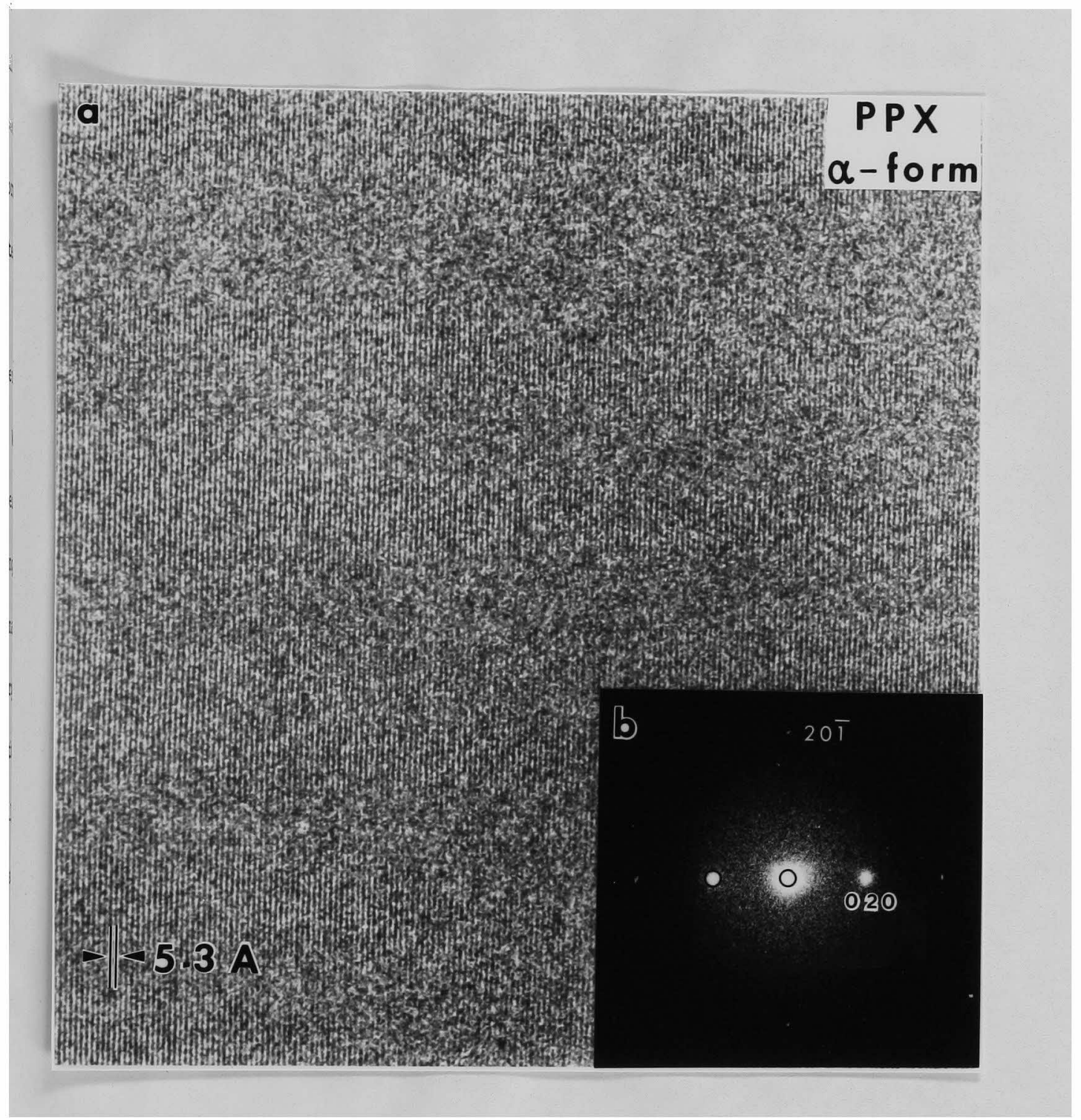

Fig.4-9 High resolution lattice image (negative contrast) of a PPX $\alpha$-form single crystal with lattice defects.

(a) Original electron micrograph taken at $500 \mathrm{KV}$.

(b) Optical diffractogram of (a).

A circle shows the hole size of the filter.

(c) Optically filtered image of (a) (reconstructed using 6 reflections $((020),(040)$, and $(20 \bar{I}))$ and the undiffracted beam ). 


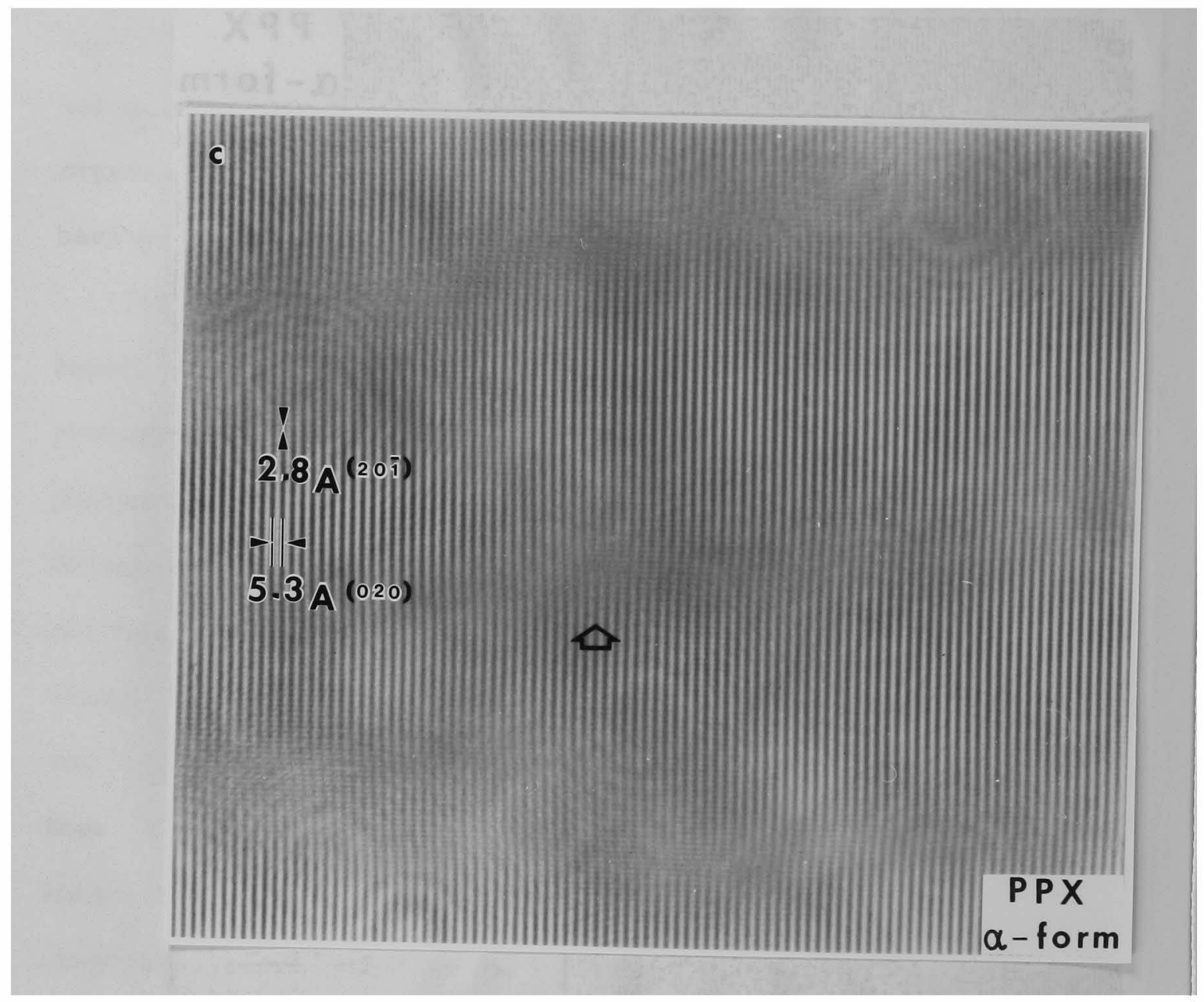

Fig.4-9 (continued) 


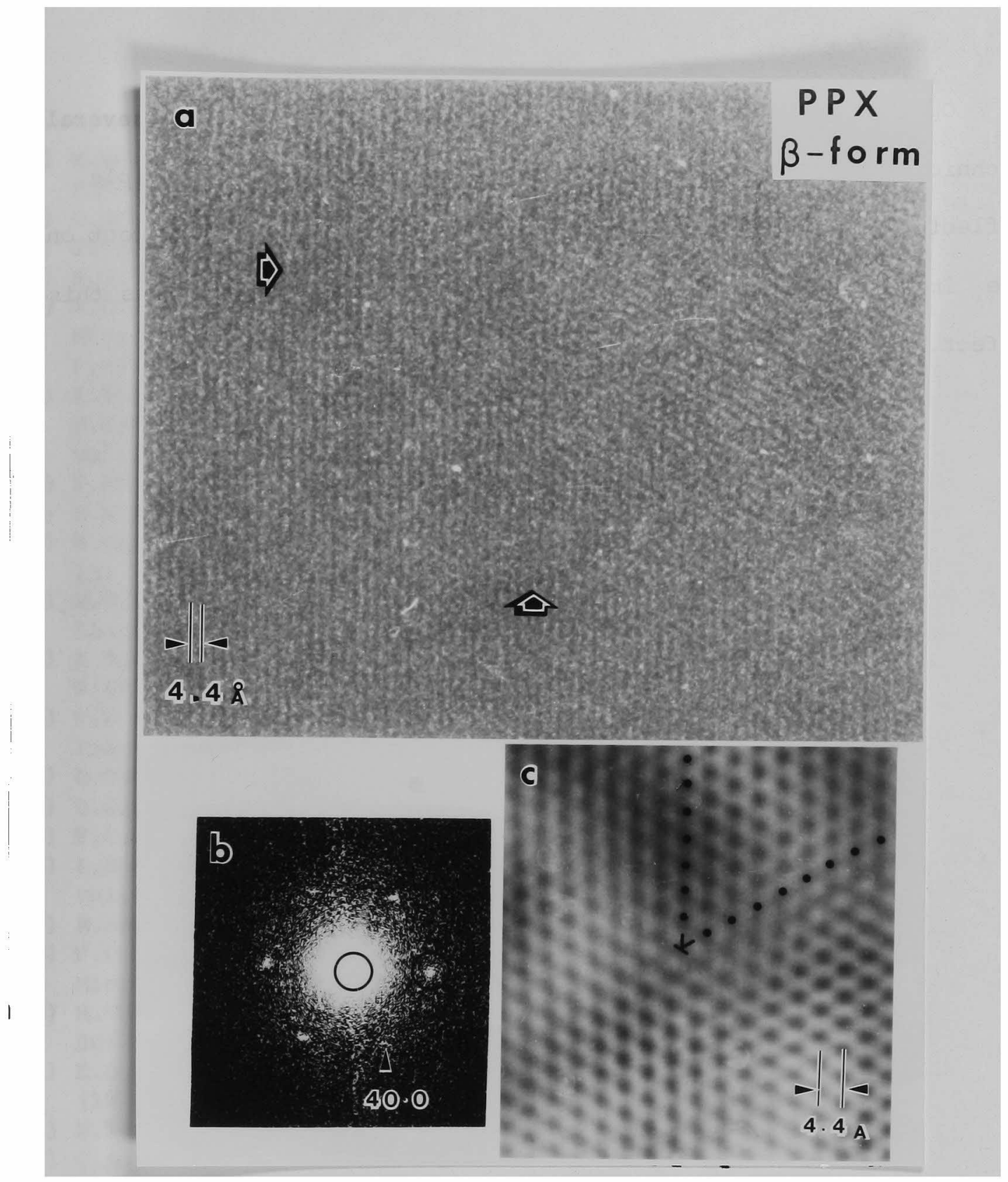

Fig.4-10 High resolution image of a PPX $\beta$-form single crystal with a lattice defect.

(a) Original electron micrograph (ab-plane projection). A lattice defect can be seen at the intersection of the trajectories of two arrows.

(b) Optical diffractogram of (a), which reveals only 6 $(40.0)$ reflections. A circle shows the hole size of the filter.

(c) Optically filtered image of (a) (reconstructed using $6(40.0)$ reflections and the undiffracted beam ). 
Optical filtering is very simple and useful, but several 87-89

technical problems still remain. For example the multiple reflection of light between lenses causes the interference effect on the image. We put lenses at long intervals to suppress this effect. 
References

1) M.Born \& E.Wolf, "Principles of Optics", 5-th ed., Pergamon Press, Chapter 8, (1975).

2) O.Scherzer, J.Appl.Phys., 20, 20 (1949).

3) J.Frank, Optik, 38, 519 (1973),

4) R.C.Valentine, Labo.Invest., 14, 1334 (1965).

5) R.C.Valentine, in "Advances in Optical and Electron Microscopy", vol.l, R.Barer \& V.E.Cosslett Ed., Academic Press, 180 (1966).

6) K.Kobayashi, E.Suito, N.Uyeda, M.Watanabe, T.Yanaka, T.Etoh, H.Watanabe \& M.Moriguch, Proc.8-th Int.Congr.EM, Canberra, vol.1, 30 (1974).

7) Y.Fujiyoshi, Denken(Electrn-Microsc.), 15, $72(1980)$.

8) G.W.Stroke \& M.Halioua, Optik, 35, 50(1972).

9) H.Okuyama, Y.Ichioka \& T.Suzuki, Japan.J.Appl.Phys., $13,280(1974)$.

10) W.O.Saxton, "Computer Techniques for Image Processing in Electron Microscopy", Academic Press, (1978).

11) A.Tonomura, T.Matsuda \& J.Endo, Japan.J.Appl.Phys., 18, 9 (1979).

12) P.W.Wawkes Ed., "Computer Processing of Electron Microscope Images", Springer-Verlag, (1980).

13) D.Gabor, Proc.Roy.Soc., A-197, 454 (1949).

14) D.Gabor, Proc.Phys.Soc., B-64, 449 (1951).

15) F.C.Billingsley, Appl.Optics, 9 , 289 (1970).

16) R.Nathan, in "Advances in Optical and Electron microscopy", vol.4, R.Barer \& V.E.Cosslett Ed., Academic Press, 85 (1971).

17) R.Bernstein, IBM.J.Res.Develop., 20, 40 (1976).

18) F.C.Billingsley, in "Advances in Optical and Electron Microscopy", vol.4, R.Barer \& V.E.Cosslett Ed., $127(1971)$.

19) M.Tsuji, S.Isoda, M.O'hara, K.Katayama \& K.Kobayashi, Bull.Inst.Chem.Res., Kyoto Univ., 55, 237(1977).

20) E.O.Brigham, "The Fast Fourier Transform", Prentice-Hall, (1974).

21) W.L.Bragg, Nature, 143, 678(1939).

22) C.A.Taylor \& H.Lipson, "Optical Transforms", G.Bell \& Sons, (1964).

23) H.Lipson ed., "Optical Transforms", Academic Press, (1972).

24) A.R.Stokes, Acta Cryst., 8, 27 (1955).

25) J.Luis \& M.Amorós, "Molecular Crystals : their transforms and diffuse scattering", John Wiley \& Sons, (1968).

26) T.R.Welberry \& R.Galbraith, J.Appl.Cryst., 6, 87 (1973) .

27) G.Bodor \& G.Samay, J.Polymer Sci.:Polym.Sump., 42, 768 (1973).

28) D.G.Fedak, T.E.Fischer \& W.D.Robertson, J.Appl.Phys., $39,5658(1968)$.

29) R.Bergsten, J.Opt.Soc.,America, 64, 1309 (1974). 
30) A.Kawaguchi, M.O'hara \& K.Kobayashi, J.Macromol.Sci.-Phys., B-16, 193(1979).

31) N.Uyeda, Denken(Electron-Microsc.), 15, 59(1980).

32) A.Klug \& J.E.Berger, J.Mol.Biol., 10, 565 (1964).

33) W.Longley, ibid., 30, $323(1967)$.

34) F.Thon \& B.M.Siegel, Ber.Bunsenges.physk.Chem., 74, 1116 (1970).

35) F.Thon, in "Electron Microscopy in Materials Science", Ed. by U.Valdré, Academic press, 571 (1971).

36) B.V.Johansen, Micron, $\underline{3}, 256$ (1972).

37) R.W.Horne, J.M.Hobart \& I.P.Ronchetti, Micron, 5, 233 (1975).

38) R.Sinclair, R.Gronsky \& G.Thomas, Acta Metall., 24, 789 (1976).

39) T.Tanji \& H.Hashimoto, Acta Cryst., A-34, 453(1978).

40) H.J.Pincus, in "Advances in Optical and Electron Microscopy", vol.7, V.E.Cosslett \& R.Barer Ed., Academic press, 17(1978).

41) R.Hosemann \& S.N.Bagchi, "Direct Analysis of Diffraction by Matter", North-Holland, (1962).

42) O.L.Krivanek, S.Isoda \& K.Kobayashi, J.Microsc., 111, 279 (1977) .

43) A.Klug \& D.J.DeRosier, Nature, 212, 29 (1966).

44) J.H.Hitchborn \& G.J.Hills, Virology, 35, 50 (1968).

45) S.Boseck \& H.Hager, Optik, 28, $602(1968 / 69)$.

46) D.J.DeRosier \& A.Klug, J.Mol.Biol., 65, 469(1972).

47) T.Mulvey, J.Microsc., 98, 232 (1972).

48) R.C.Warren \& R.M.Hicks, Micron, 4, 257(1973).

49) R.W.Horne \& R.Markham, "Electron Diffraction and Optical Diffraction Techniques", North-Holland, 327 (1973).

50) H.P.Erickson, J.Cell.Biol., 60, 153(1974).

51) U.Aebi, P.R.Smith, J.Dubochet, C.Henry \& E.Kellenberger, J.Supramol.Struct., $1,498(1974)$.

52) A.C.Steven, U.Aebi \& M.K.Showe, J.Mol.Biol., 102, 373(1976).

53) H.Hashimoto, H.Endoh \& T.Tanji, Proc.US-Japan Seminar, HVEM, Honolulu, (1976).

54) F.P.Ottensmeyer, J.W.Andrew, D.P.Bazett-Jones, A.S.K.Chan \& J.Hewitt, J.Microsc., 109, 259(1977) .

55) M.Osumi, M.Nagano \& M.Yanagida, J.Electron Microsc., 28 , 301 (1979).

56) K.Shimamura, J.Macromol.Sci.-Phys., B-16, 213 (1979).

57) D.J.DeRosier \& A.Klug, Nature, 217, 130(1968).

58) R.A.Crowther, L.A.Amos, J.T.Finch, D.J.DeRosier \& A.Klug, Nature, 226, 421(1970). $59)$ P.B.Moore, H.E.Huxley \& D.J.DeRosier, J.Mol.Biol., 50,
$279(1970)$.

60) N.A.Kiselev \& A.Klug, J.Mol.Biol., 40, 155 (1969).

61) R.A.Crowther, D.J.Derosier \& A.Klug, Pro.ROy.Soc., London, $A-317,319(1970)$.

62) A.Klug \& R.A.Crowther, Nature, 238, 435 (1972).

63) L.A.Amos, J.Microsc., 100, $143(1974)$. 
64) P.R.Smith, U.Aebi, R.Josephs \& M.Kessel, J.Mol.Biol., 106, 243(1976).

65) B.K.Vainshtein, in "Advances in Optical and Electron Microscopy", vol.7, V.E.Cosslett \& R.Barer Ed., Acadmic Press $282(1978)$.

66) D.L.Misell, "Image Analysis, Enhancement and Interpretation", ( Practical Methods in EM, vol.7, A.M.Glauert Ed.), North-Holland, (1978).

67) P.K.Luther \& J.M.Squire, J.Mol.Biol., 14l, 409 (1980).

68) R.W.Horne, J.Microsc., 113, 241 (1979).

69) H.P.Erickson \& A.Klug., Ber.Bunsenges.phys.Chem., 74, 1129 (1970).

70) J.Frank, Biophys.J., 12, 484 (1972).

71) J.Frank \& L.Al-Ali, Nature, 256, 376 (1975).

72) P.N.T.Unwin \& R.Henderson, J.Mol.Biol., 94, 425 (1975).

73) D.L.Misell \& R.E.Burge, J.Microsc., 103, 195 (1975).

74) N.Uyeda \& K.Ishizuka, J.Electron Microsc. , 24, 65 (1975).

75) R.D.B.Fraser, T.P.MacRae, E.Suzuki \& C.L.Davey, J.MicrosC., $108,343(1976)$.

76) A.N.Barrett, I.D.J.Burdett \& K.A.Paton, ibid., 113, 131 (1978).

77) L.Fang-Hua \& F.Hai-Fu, Acta Phys.Sin., 28, $276(\overline{1979})$.

78) E.L.O'Neill, IRE Trans.Inf.Theory, IT-2,56(1956).

79) H.Thiry, Appl.Optics, 3, 39(1964).

80) A.Kozma \& D.L.Kelly, ibid., 4 , 387(1965).

81) D.McLachlan,Jr., Proc.N.A.S., 44, 948 (1958).

82) R.Markham, J.H.Hitchborn, G.J.Hills \& S.Frey, Virology, $22,342(1964)$.

83) F.P.Ottensmeyer, E.E.Schmidt \& A.J.Olbrecht, Science, $179,175(1972)$.

6) 84) J.M.Cowley, "Diffraction Physics", North-Holland, Chapter 7, (1975).

85) H.P.Erickson, Proc.8-th Int.Congr.EM, Canberra, vol.1, 310 (1974).

86) R.D.B.Fraser \& G.R.Millward, J.Ultrastruct.Res., 31, $203(1970)$.

87) W.L.Bragg \& A.R.Stokes, Nature, 156, $332(1945)$.

88) C.A.Taylor \& J.K.Ranniko, J.Microsc., 100, 307 (1974).

89) Y.Masuda \& R.Ueda, J.Cryst.Soc.Japan, 18, 298(1976).

90) N.Uyeda, Y.Fujiyoshi \& K.Ishizuka, Proc.US-Japan Seminar,HVEM, Honolulu, (1976).

91) Y.Fujiyoshi, K.Ishizuka \& N.Uyeda, J.Electron Microsc., 26, 47 (1977).

92) W.Hoppe, Acta Cryst., A-26, 414 (1970).

93) I.A.M.Kuo \& R.M.Glaeser, Ultramicrosc., 1,53 (1975).

94) K.Kondo, N.Nakajima, Y.Ichioka \& T.Suzuki, Oyobutsuri, 47, $1140(1978)$.

95) B.T.M.Willis, Proc.Roy.Soc.London, A-239, 184 (1957).

96) B.K.Mathur, B.K.Samantaray \& G.B.Mitra, Acta Cryst., A-34, 284 (1978). 
Chapter 5 : Crystal structure analysis of PPX B-form crystal

Since Brown \& Farthing discovered $\alpha$ - and $\beta$-modifications of PPX crystals by X-ray diffraction, $2-10,26,27$ investigated PPX crystals. The crystal systems and unit cell dimensions are shown in Table $5-1(a)$ for the $\alpha$-form and Table 5-1(b) for the $\beta$-form. The PPX $\alpha$-form crystals were already analyzed by Iwamoto \& Wunderlich by $\mathrm{x}$-ray diffraction and their result is shown in Fig.2-6 and Table 2-1 in Chapter 2. They adopted the crystal system and unit cell dimensions reported by kubo \& Wunderlich (1972). Their calculated density $1.180 \mathrm{~g} / \mathrm{cm}^{3}$ is in good agreement with the observed density of $\alpha$-form crystals. Their value for the c-axis (molecular axis), namely the identity period is $6.55 \AA$ corresponding to the extended, trans-zigzag conformation (Fig.5-1). 
Reported crystallographic data of PPX crystals
(a) ; $\alpha$-form,
(b) ; $\beta$-form

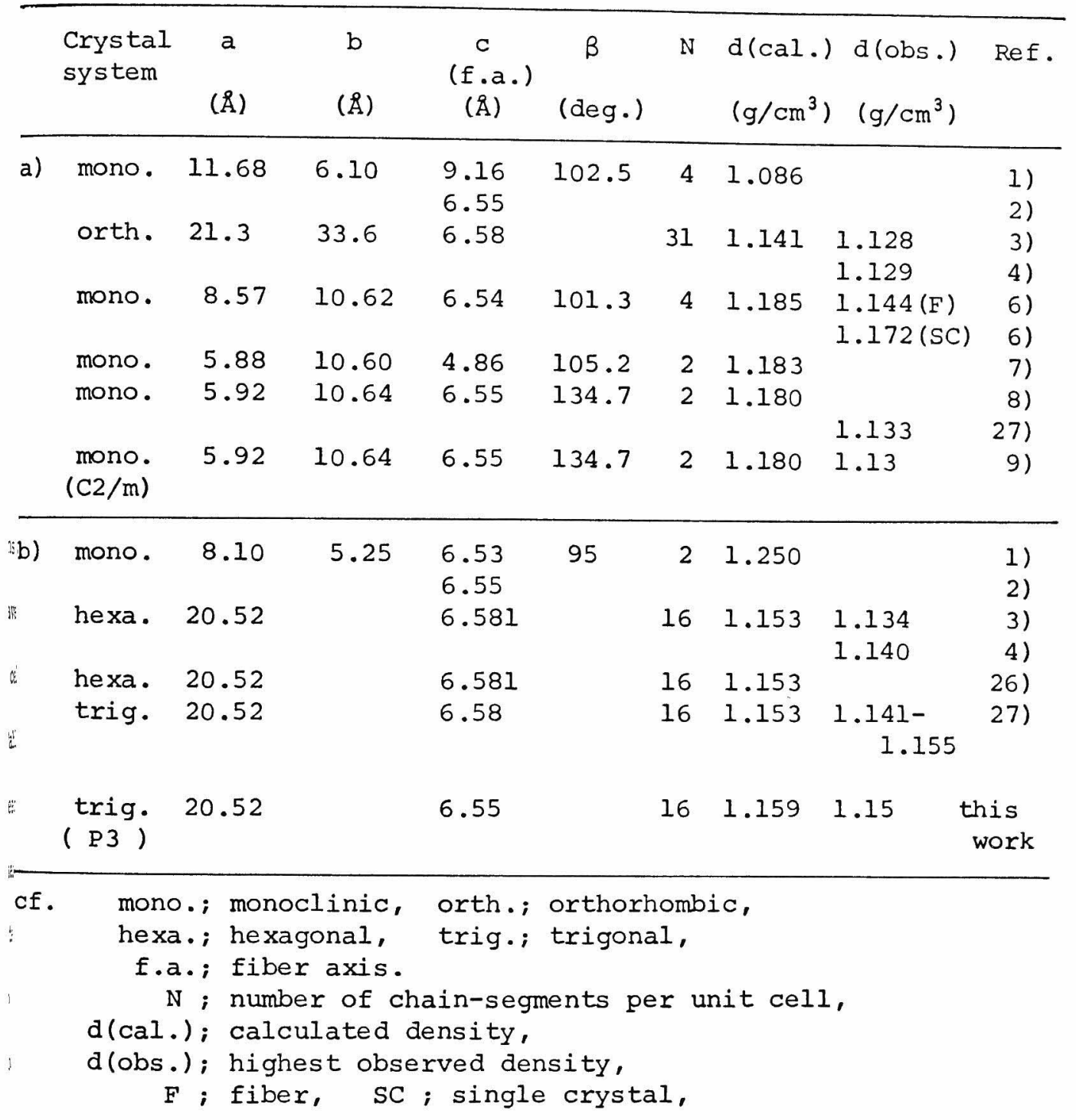



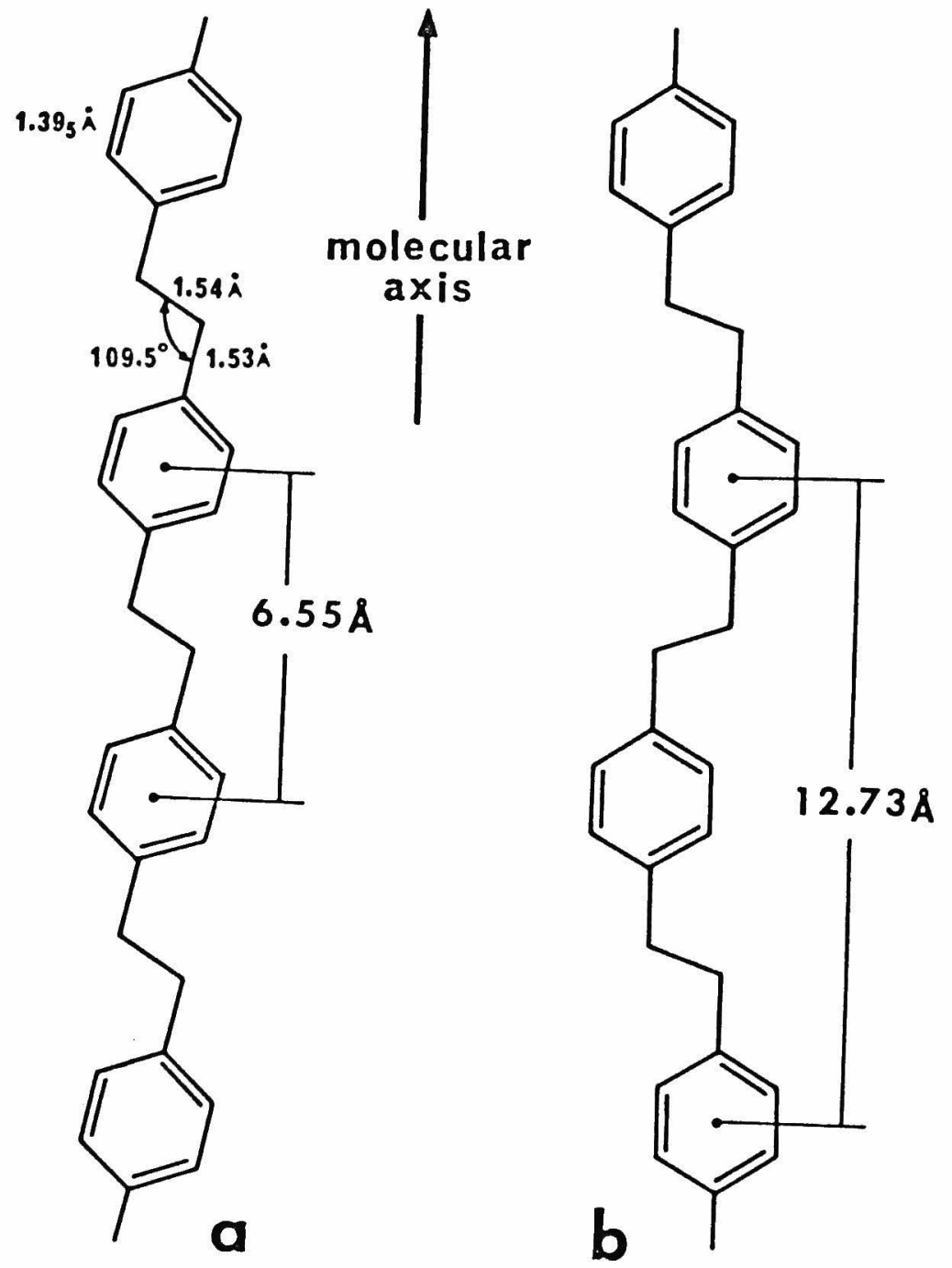

Fig.5-1 Molecular conformation in PPX crystals.

(a) ; trans zigzag conformation, which is likely to be in PPX crystals (fiber period; $6.55 \AA$ )

(b) ; cis conformation (fiber period; $12.73 \AA$ ). 
Two crystal systems were proposed for the PPX $\beta$-form crystal 3

( see Table 5-1(b) ). Niegisch studied optical properties of the $\beta$-form as well as the crystal structure, and observed the $\beta$-form single crystals grown from $\alpha$-chloronaphthalene solution with an electron microscope. He proposed the crystal system and unit cell dimensions based on his experimental results. Taking into account his results and our electron/X-ray diffraction data as well as the already analysed crystal structure of the $\alpha$-form ( especially the dimension of c-axis, together, we proposed the crystal system and

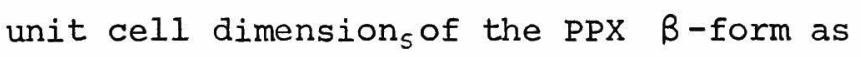

hexagonal or trigonal; $a=20.52 \AA, c($ molecular axis) $=6.55 \AA$.

A density of $1.15 \mathrm{~g} / \mathrm{cm}^{3}$ was obtained in our experiment as the highest observed density of the PPX $\beta$-form, whereas $1.134 \mathrm{~g} / \mathrm{cm}^{3}$ and $1.140 \mathrm{~g} / \mathrm{cm}^{3}$ were reported by Niegisch ${ }^{4}$ ( see Table 5-1(b) ). In either case, it is concluded from the density and lattice dimensions that a unit cell must contain 16 monomer units, that is to say, 16 polymer chain-segments ( the length of a "chain-segment" is the fiber period, ought to be contained in a unit cell. This has made the structure analysis of the $\beta$-form crystal complicated. Prior to the structure analysis of the PPX $\beta$-form crystal, we classified the polymer crystals belonging to the hexagonal or trigonal system into three groups as follows:

Group 1 : Each chain has 3- or 6-fold axis or screw axis. Group 2 : Each chain has cylindrical or pseudo-cylindrical 
symmetry. In this case, a unit cell is made up of only one molecular segment.

Group 3 : An integral multiple of 3 identical chain-segments constitute a unit cell, though the molecular chain has no symmetry features of Group 1 or 2 .

Some examples are shown in Table 5-2. The PPX $\beta$-form crystal which does not suit any of the above-mentioned prerequisites is an unusual example, and the crystal structure has not yet been analysed.

5-1 Determination of the PPX $\quad$-form crystal structure in the projection on the ab-plane

As shown in Chapter 3, we were able to obtain the high , resolution electron micrograph of a PPX $\beta$-form single crystal with JEM-500. The optical diffraction pattern of this micrograph closely resembles the electron diffraction pattern of a PPX $\beta$-form single crystal. The micrograph, which was already image-processed and improved in the $\mathrm{S} / \mathrm{N}$ ratio, is shown again in Fig.5-2(a). Each black spot in Fig.5-2(a) is to represent the projection of one molecular chain on the ab-plane. The possibility of obtaining such an image-contrast is confirmed by a computer-simulated image under nearly optimum defocus based on the assumed model structure. Thus 
Table 5-2

Grouping of polymer crystals

belonging to hexagonal or trigonal system

\begin{tabular}{|c|c|c|c|c|c|}
\hline Group & Polymer & $\begin{array}{l}\text { ystal } \\
\text { stem }\end{array}$ & $\begin{array}{l}\text { Molecular } \\
\text { conformation }\end{array}$ & $\mathrm{N}$ & Ref. \\
\hline \multirow[t]{7}{*}{1} & it-Polypropylene, $\beta$-form & $\mathrm{H}$ & Helix $(3 / 1)$ & 9 & 13) \\
\hline & Polyoxymethylene & $\mathrm{T}$ & Helix $(9 / 5)$ & 1 & 14) \\
\hline & it-Polystyrene & $\mathrm{T}$ & Helix $(3 / 1)$ & 6 & 15) \\
\hline & it-Poly-1-butene & $\mathrm{T}$ & Helix $(3 / 1)$ & 6 & 16) \\
\hline & Poly-1,3-dioxolane, form-III & $\mathrm{H}$ & Helix $(6 / 1)$ & 3 & 17) \\
\hline & it-Poly (vinyl methyl ether) & $\mathrm{T}$ & Helix $(3 / 1)$ & 6 & 18) \\
\hline & $\begin{array}{l}\text { Polytetrafluoroethylene } \\
\text { ( above } 19^{\circ} \mathrm{C} \text { ) }\end{array}$ & $\mathrm{T}$ & Helix(15/7) & 1 & 19) \\
\hline \multirow[t]{3}{*}{2} & $\begin{array}{l}\text { Polytetrafluoroethylene } \\
\text { ( below } 19^{\circ} \mathrm{C} \text { ) }\end{array}$ & p.H & Helix $(13 / 6)$ & 1 & 19) \\
\hline & Polychlorotrifluoroe thylene & $\mathrm{p} \cdot \mathrm{H}$ & $\begin{array}{l}\operatorname{Helix}(16.8 / 1) \\
\text { (average) }\end{array}$ & 1 & 20) \\
\hline & Nylon $77, \gamma$-form & p.H & $(1 / 0)$ & 1 & 21) \\
\hline 3 & Polyoxacyclobutane, form-II & $\mathrm{T}$ & Glide-type $(2 / 0)$ & 9 & 22) \\
\hline
\end{tabular}

cf. $N$; number of chain-segments per unit cell,

$\mathrm{H}$; hexagonal, $\mathrm{T}$; trigonal, p.H ; pseudo-hexagonal.

See References ((23)-(25)) for other examples. 


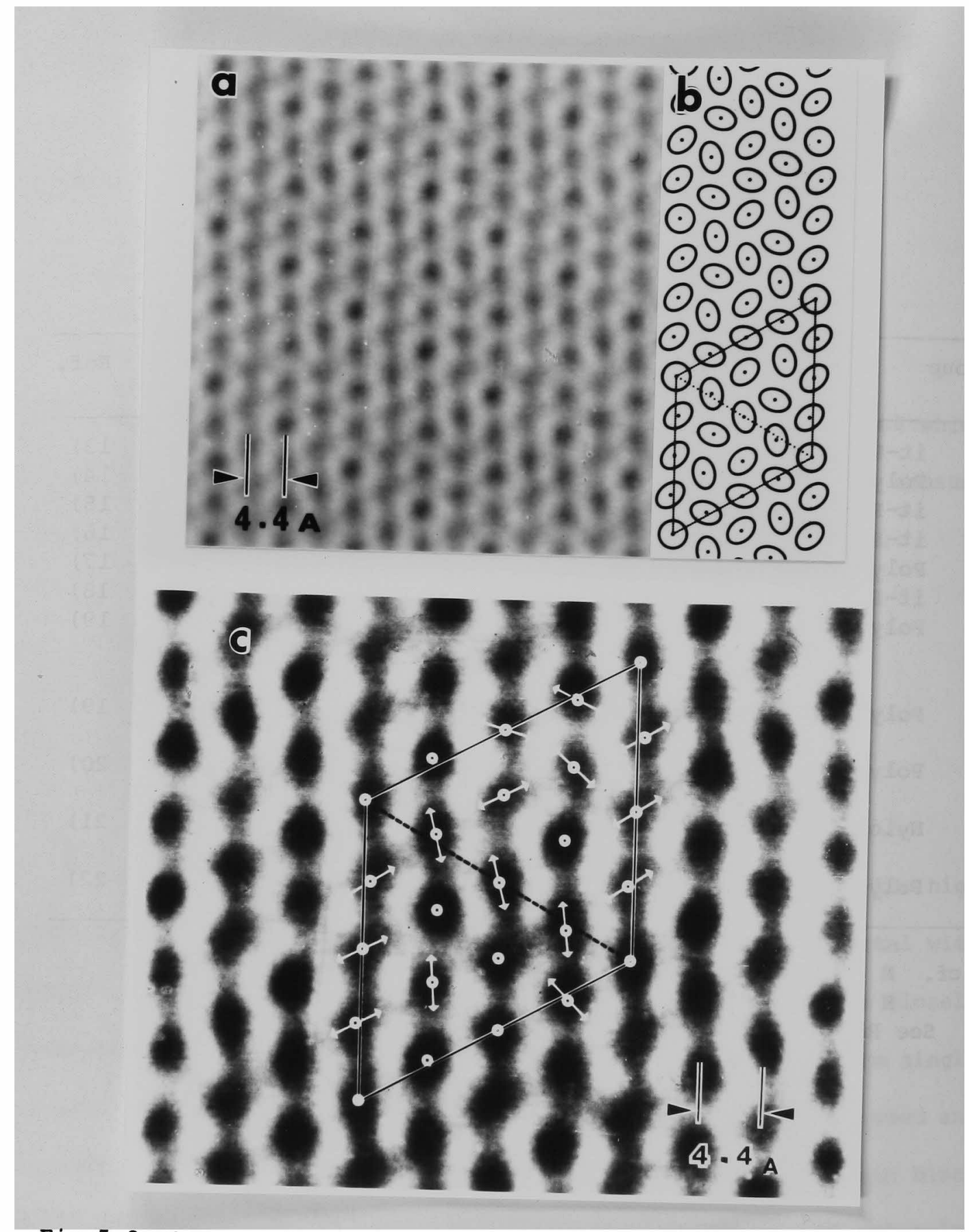

Fig.5-2 High resolution image of a PPX $\beta$-form single crystal.

(a) Optically filtered image (Chapter 4).

(b) Structure model as the result of analysis by electron diffraction.

(c) Enlarged photograph of (a). An arrow shows the orientation of a ellipse which can be defined in this
photograph. 
re have succeeded in direct imaging of the individual molecular hains of PPX. Some of the spots in this micrograph seem to be :1liptic. Figure 5-2(c) shows the directions of major axes of such :lipses, where the arrows correspond to the molecular orientations in the ab-plane projection.

The significance of the processed image (Fig.5-2(a)) is its :nhancement of the features of the molecular arrangement. Here the Iutual positions of the molecular centers in the projection on the ib-plane are observed. The feature of the arrangement of molecular :enters seems to be represented in terms of the 2-dimensional jattern shown in Fig.5-3. This pattern is called "Chidori" ( a yeneric name to some kinds of small birds in Japan ), which is one of the repeating patterns in dyeing and industrial arts in Japan. Though the 2-dimensional space group of "Chidori" is p3, that of the nolecular arrangement in the PPX $\beta$-form crystal is $p 6$ which has no conditions limiting possible reflections. Indeed, the electron diffraction pattern of a PPX $\beta$-form single crystal has neither systematic absences of reflections nor symmetry planes but only shows 6-fold rotational symmetry, as described in Chapter 2.

We first assumed a 2-dimensional structure model of the PPX B-form crystal so as to satisfy the symmetry of $p 6$, taking van der 'Waals' radii and the electron microscopic image (Fig.5-2(a)) into account. The mutual positions and orientations of 16 molecules i( see Fig.5-4) were changed so as to be consistent with the 


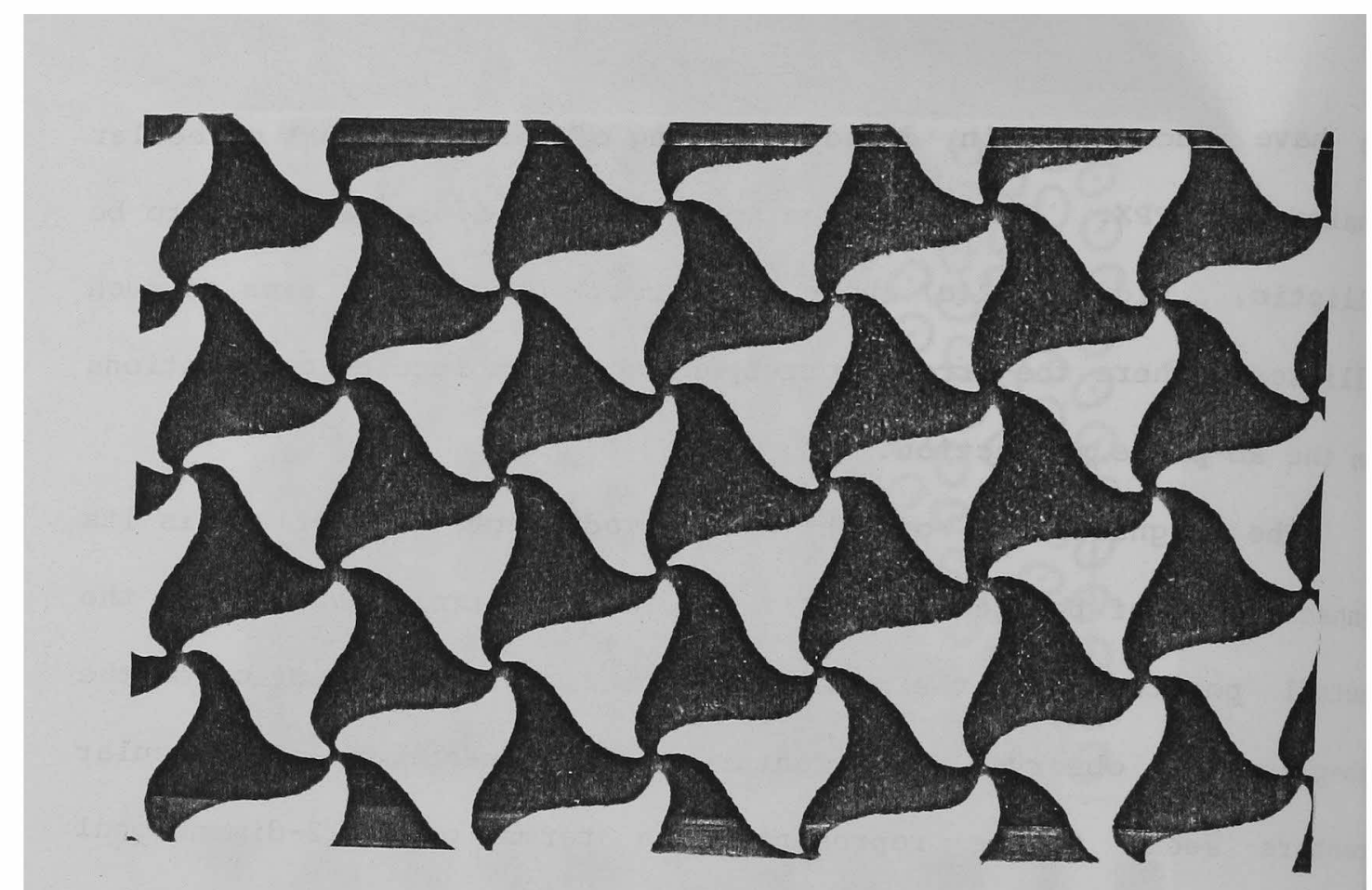

Fig.5-3 "Chidori" pattern ( 2-dimensional space group ; p3).

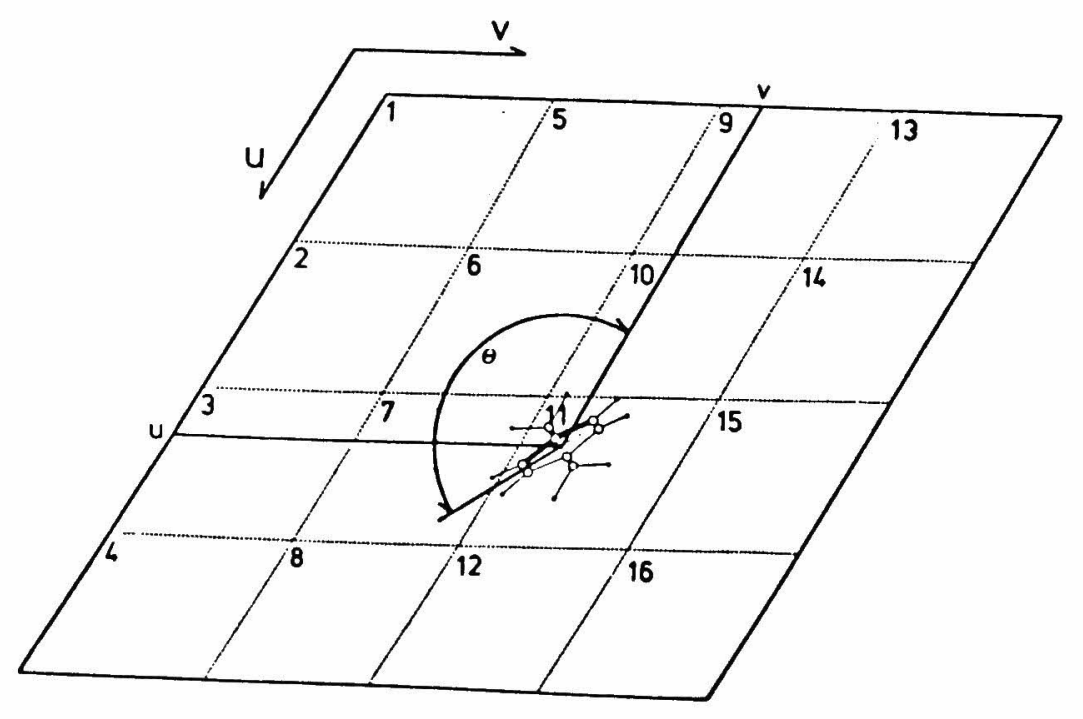

Fig.5-4 Parameters for the crystal structure analysis. 
electron diffraction pattern (Fig.5-5). At the time of calculation the orientation of the molecule at the origin of a unit cell was assigned statistically to one of three equivalent orientations to satisfy the symmetry of p6 ( All the calculations in the present study were carried out with an electronic computer FACOM-M160AD in the Institute for Chemical Research, Kyoto University ).

We measured the electron diffraction intensity from a PPX $\beta$-form single crystal with the microdensitometer mentioned in Chapter 4. The refinement of the structure analysis was attempted by the least square method over the electron diffraction intensity data thus obtained. 25 reflections were used in our analysis. The calculated structure factor $\mathrm{F}_{\mathrm{cal}}$. for $500 \mathrm{KV}$ electrons and the square-root of the observed intensity, $\sqrt{I_{\text {obs}}}$, are shown in Table 5-3(b). A PPX "single crystal" is a mosaic crystal which gives a spot-pattern (so-called "N-pattern") in electron diffraction. Therefore we used the $1 / \alpha_{h k 0}$ as the correction factor corresponding to Lorentz factor in X-ray diffraction, where $\mathrm{d}_{\mathrm{hk0}}$ is the (hk0) spacing of the crystal. In this case, the reliability factor $\mathrm{R}$ was 31\%, and the isotropic temperature factor $B$ was $7.6 \AA^{2}$. The molecular conformation of the PPX $\beta$-form took after that of the $\alpha$-form ( Fig.2-6 and Table 2-1 in Chapter 1 ) since the reliability factor $R$ was minimized with this conformation. Thus the structure model is deduced as shown in Fig.5-6 and Table 5-3(a). If we represent the molecule at the origin of a unit cell with a circle 
Table 5-3(a)

Resulting parameters analysed by electron diffraction

\begin{tabular}{cllll}
\hline $\begin{array}{c}\text { No. of } \\
\text { molecule }\end{array}$ & $\mathrm{u}$ & $\mathrm{v}$ & $\mathrm{w}$ & $\begin{array}{c}\theta \\
\text { (degree) }\end{array}$ \\
\hline 1 & 0.0 & 0.0 & - & 147 \\
2 & 0.252 & 0.031 & - & 138 \\
3 & 0.5 & 0.0 & - & 139 \\
7 & 0.518 & 0.270 & - & 70 \\
\hline
\end{tabular}

cf. $u, v, w$; fractional coordinates ( see Fig.5-4).

Table 5-3(b)

Calculated structure factor $\mathrm{F}_{\mathrm{cal}}$. for $500 \mathrm{KV}$ electrons and the square root of the observed intensity, $\sqrt{I_{\text {obs. }}} \quad\left(\mathrm{R}=31 \%, \quad \mathrm{~B}=7.6 \AA^{2}\right)$

\begin{tabular}{|c|c|c|c|c|c|}
\hline $\mathrm{H}$ & $\mathrm{K}$ & L & $\left(\begin{array}{l}\mathrm{d} \\
\AA\end{array}\right)$ & $\mathrm{F}_{\mathrm{cal}}$ & $\sqrt{I_{\text {obs }}}$ \\
\hline 1 & 0 & 0 & 17.77 & -14.52 & 38 \\
\hline 2 & 0 & 0 & 8.89 & -23.42 & 34 \\
\hline 3 & 0 & 0 & 5.92 & -32.42 & 18 \\
\hline 4 & 0 & 0 & 4.44 & 344.40 & 344 \\
\hline 5 & 0 & 0 & 3.55 & -10.15 & 24 \\
\hline 6 & 0 & 0 & 2.96 & -29.88 & 30 \\
\hline 7 & 0 & 0 & 2.54 & -9.40 & 9 \\
\hline 8 & 0 & 0 & 2.22 & 48.07 & 62 \\
\hline 1 & 1 & 0 & 10.26 & 19.20 & 20 \\
\hline 2 & 1 & 0 & 6.72 & -35.82 & 16 \\
\hline 3 & 1 & 0 & 4.93 & 38.86 & 66 \\
\hline 4 & 1 & 0 & 3.88 & 52.87 & 104 \\
\hline 5 & 1 & 0 & 3.19 & 6.08 & 42 \\
\hline 6 & 1 & 0 & 2.71 & 9.41 & 12 \\
\hline 7 & 1 & 0 & 2.35 & -1.35 & 10 \\
\hline 1 & 2 & 0 & 6.72 & -64.43 & 20 \\
\hline 2 & 2 & 0 & 5.13 & 10.74 & 22 \\
\hline 3 & 2 & 0 & 4.08 & 22.10 & 64 \\
\hline 4 & 2 & 0 & 3.36 & 78.91 & 76 \\
\hline 5 & 2 & 0 & 2.85 & -31.59 & 6 \\
\hline 6 & 2 & 0 & 2.46 & -27.84 & 26 \\
\hline 1 & $3 c$ & 0 & 4.93 & -76.49 & 86 \\
\hline 2 & $3 c$ & 0 & 4.08 & 26.64 & 64 \\
\hline 3 & $3 c$ & 0 & 3.42 & -14.11 & 28 \\
\hline 4 & 30 & 0 & 2.92 & -14.48 & 16 \\
\hline
\end{tabular}




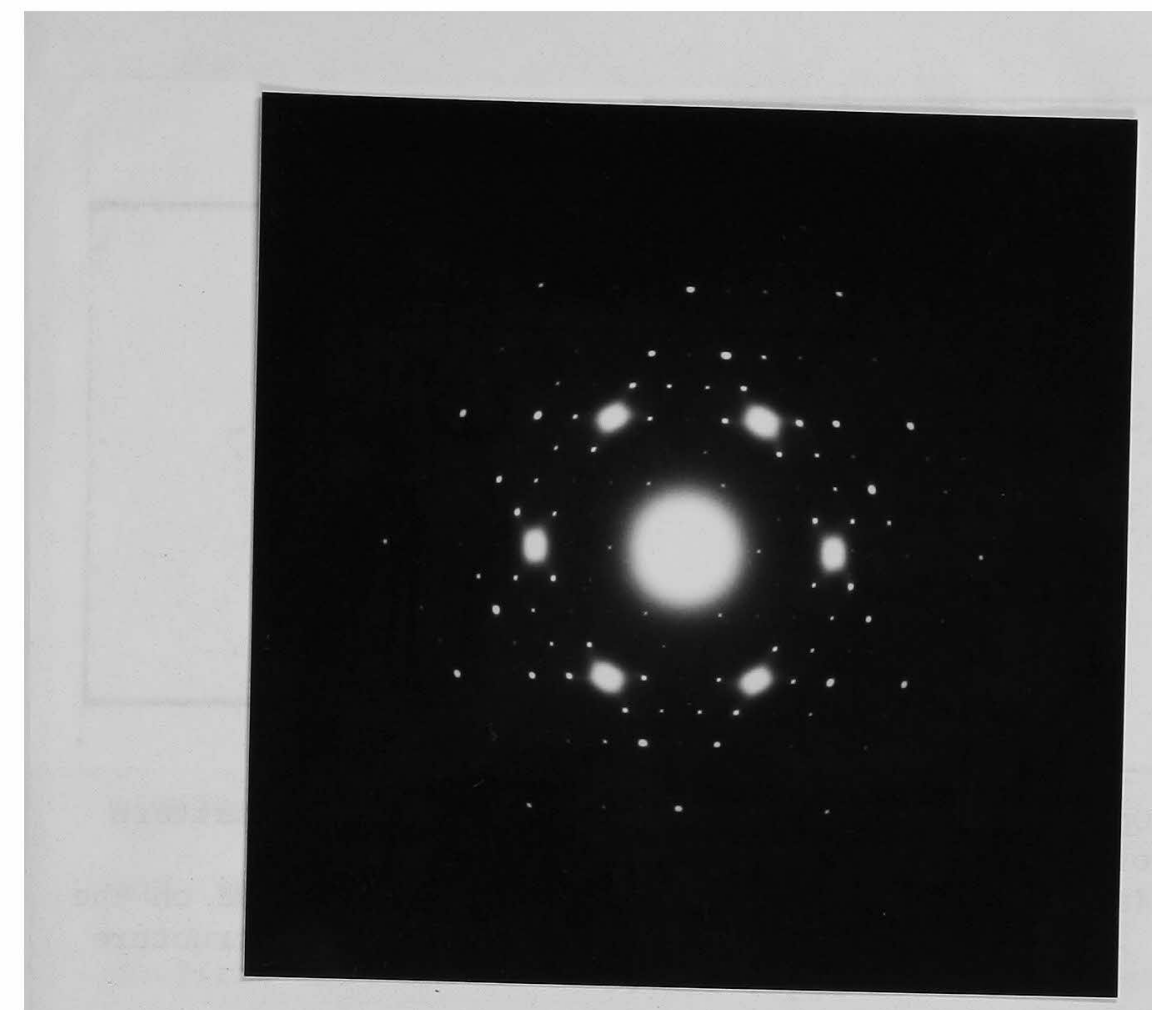

Fig.5-5 Selected-area electron diffraction pattern of a PPX $\beta$-form single crystal ( $500 \mathrm{KV}$ electrons).
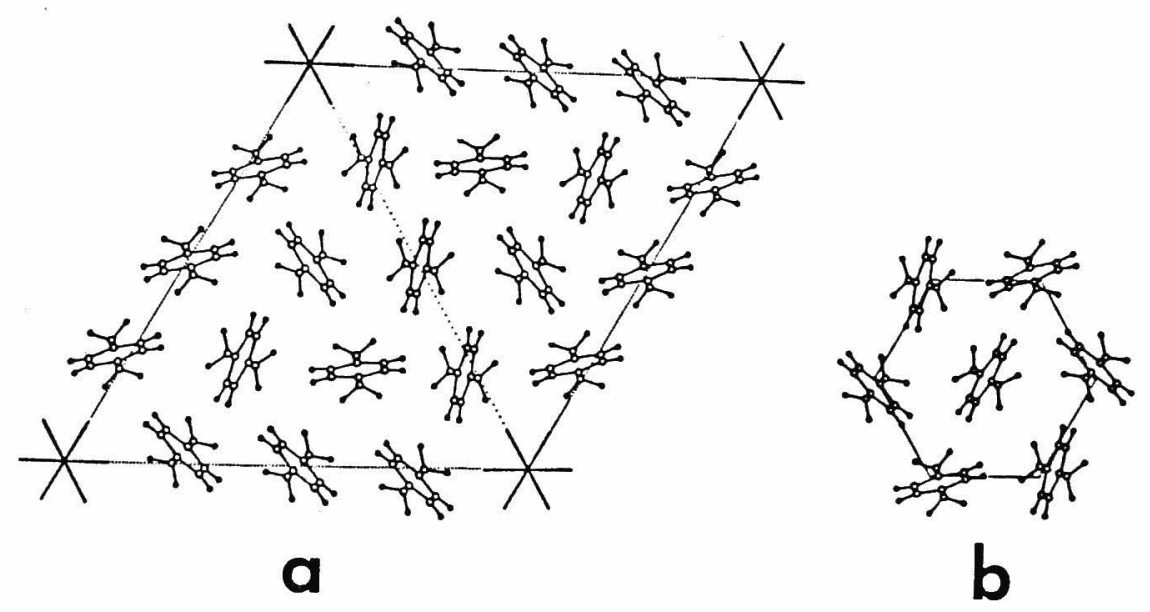

Fig.5-6 Two-dimensional structure corresponding to Table 5-3(a). ( analysed by electron diffraction ).

(b) ; one of 3 equivalent molecular orientations at the origin of a unit cell ( $*$ in $(a))$. 


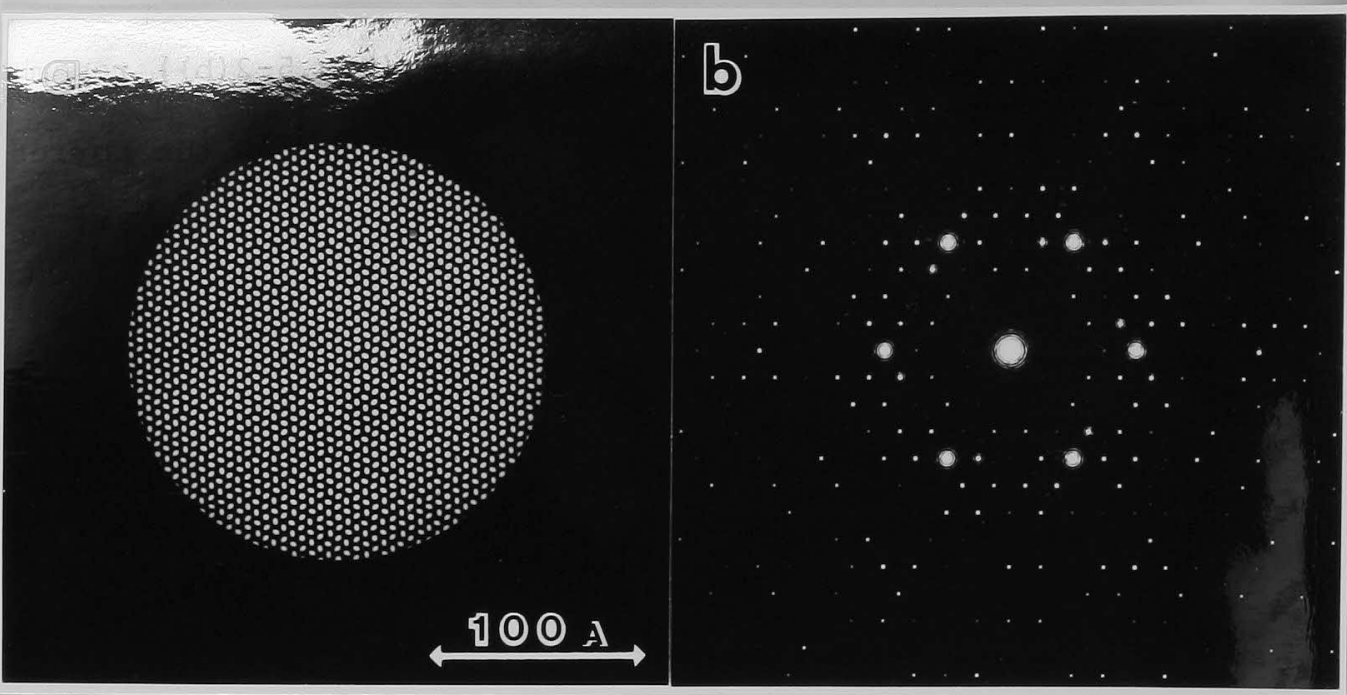

Fig.5-7 Optical analogy to the electron diffraction pattern of a PPX $\beta$-form single crystal.

(a) Mask for optical transform, which was made on the basis of Table $5-3(a)$ and has the same structure as that of Fig.5-2(b).

(b) Optical transform of (a).

Fig.5-8 X-ray diffraction photograph of a PPX $\beta$-form specimen with a cylindrical camera (camera radius; $35.0 \mathrm{~mm}$ ). 
and others with ellipses, this model (Fig.5-2(b)) resembles the micrograph (Fig.5-2(a)) fairly well, and some of the corresponding ellipses and spots in these figures coincide in position and in orientation with each other.

Moreover we made an optical mask having the same structure as this model and performed the optical transform of the mask in order to ascertain our result. Figures 5-7(a) and (b) show the optical mask and the corresponding optical diffraction, respectively. The similarity between the $500 \mathrm{KV}$-electron diffraction pattern (Fig.5-5) and the optical diffraction pattern (Fig.5-7(b)) of the model confirms that our model properly represents the crystal structure of the PPX $\beta$-form.

5-2 Crystal structure analysis of the PPX $\beta$-form by $\mathrm{x}$-ray diffraction

The X-ray diffraction intensities from a PPX specimen of the B-modification which had been elongated 6 times at $285^{\circ} \mathrm{C}$, ( the temperature is higher than the $\alpha \rightarrow \beta$ irreversible transition temperature. See Fig.2-1 in Chapter 2) were measured by an ordinary photographic method. Here monochromatized X-rays with a graphite crystal and a cylindrical camera were used. The specimen was rotated around the elongation axis during photographing. An 
example of the X-ray diffraction photographs is shown in Fig.5-8, which shows a typical fiber pattern. The camera radius is $35 \mathrm{~mm}$. The optical density of the films was measured with a mircrodensitometer, and relative intensities were calculated from density data using a curve-fitting program. Inseparable reflections were used in the lump. The reflections were indexed from the lattice constants: $a=b=20.52 \AA, \quad c$ (molecular axis) $=6.55 \AA$, and $\gamma=120^{\circ}$. The method of least squares was used for the analysis by $x$-ray diffraction.

5-2-1 Two-dimensional analysis using $\mathrm{x}$-ray equatorial reflections First of all, we analysed the 2-dimensional structure projected on the ab-plane in order to confirm the result of the analysis by electron diffraction. The 17 independent reflections on the equator were used and the resulting structure parameters were listed in Table 5-4. In this case, $R=13.5 \%$ and $B=7.6 \AA^{2}$ (isotropic). The same molecular conformation as that of the $\alpha$-form was adopted since it lowered the reliability factor; that is, benzene rings are perpendicular to the zigzag plane of $-\mathrm{CH}_{2}-\mathrm{CH}_{2}-$. Figure 5-9 shows the schematic structure model. The mutual positions and orientations of molecules are almost identical to those analysed by the electron diffraction (Fig.5-6 or Table 5-3(a)). In the 3-dimensional analysis, therefore, this structure in the ab-plane projection was fixed. 
Table 5-4

Resulting parameters analysed using $X$-ray equatorial reflections ( $R=13.5 \%, B=7.56 \AA^{2}$ )

\begin{tabular}{cllll}
\hline $\begin{array}{c}\text { No. of } \\
\text { molecule }\end{array}$ & $\mathbf{u}$ & $\mathbf{v}$ & $\mathrm{w}$ & $\begin{array}{c}\theta \\
\text { (degree) }\end{array}$ \\
\hline 1 & 0.0 & 0.0 & - & 120 \\
2 & 0.257 & 0.054 & - & 123 \\
3 & 0.5 & 0.0 & - & 120 \\
7 & 0.504 & 0.266 & - & 83 \\
\hline
\end{tabular}

cf. $u, v, w$; fractional coordinates ( see Fig.5-4).

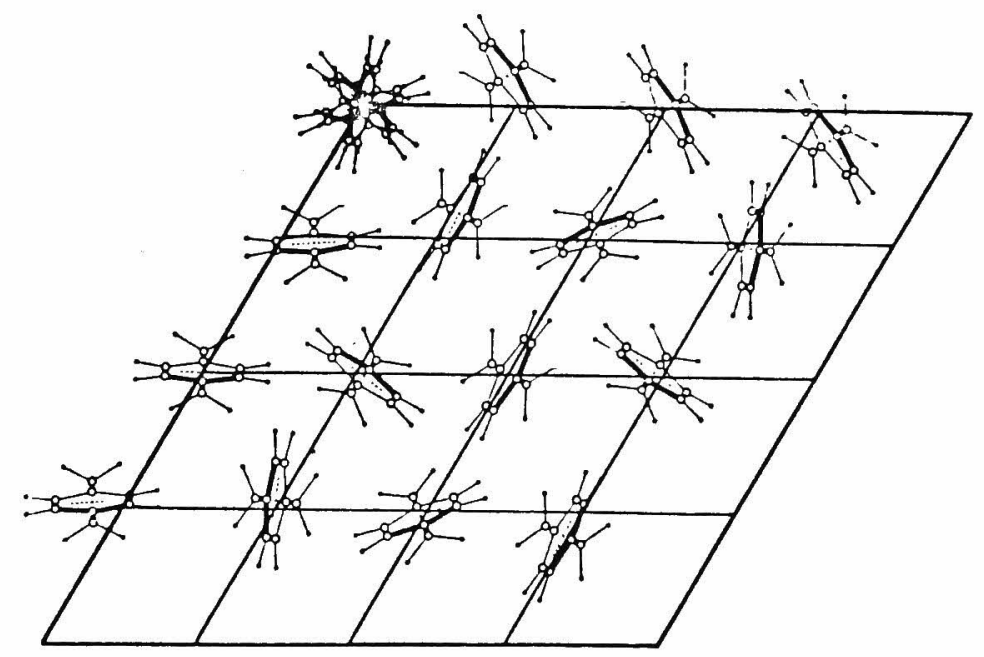

Fig.5-9 Two-dimensional structure corresponding to Table 5-4. ( analysed using $\mathrm{x}$-ray equatorial reflections). 


\section{5-2-2 Three-dimensional structure analysis}

The intensity distribution of meridional reflections of the PPX $\beta$-form crystal was close to that of the $\alpha$-form. Therefore, the corresponding atoms of individual molecular segments in a unit cell must lie on the same plane as is the case in the $\alpha$-form. This requirement should be approximately fulfilled.

The two molecular orientations ( those coincide with each other by $180^{\circ}$ rotation about the molecular axis ) can give the identical projection on the ab-plane. ( Such a problem took place also in the case of the $\alpha$-Form crystal structure analysis, and Iwamoto \& Wunderlich determined the orientation using the reflections on the first layer line.) The 3-dimensional space group of the PPX $\beta$-form crystal is to give the 2-dimensional space group $p 6$ in the projection on the ab-plane, so that it should be either $\mathrm{P} 3$ or $\mathrm{p}^{\frac{28}{3}}$. We determined the following structure whose space group is P3 (trigonal), because the structure having the P3 symmetry lowered the reliability factor than $\mathrm{P} \overline{3}$. Resulting parameters ( see Fig.5-4) are shown in Table 5-5, with the calculated structure amplitude $\left(\mid F_{\text {cal }}.\right)$ and the observed one $\left(\left|F_{\text {obs. }}\right|\right)$. In this case, $R=26.3 \%$ and $B=7.56 \AA^{2}$ (isotropic). The schematic model structure is shown in Fig.5-10. The orientation of the molecule (NO.1) at the origin of a unit cell is assigned statistically to one of three equivalent orientations in Fig.5-10. The bold lines of the molecular backbone represent the upper part of a benzene ring, in this figure. 
Table 5-5(a)

Resulting 3-dimensional parameters analysed by $\mathrm{X}$-ray diffraction ( 3 -dimensional space group ; $\mathrm{P} 3$ )

\begin{tabular}{cllcc}
$\begin{array}{c}\text { No. of } \\
\text { molecule }\end{array}$ & $\mathrm{u}$ & $\mathrm{v}$ & $\mathrm{w}$ & $\begin{array}{c}\theta \\
\text { (degree) }\end{array}$ \\
\hline 1 & 0.0 & 0.0 & 0.0 & 99 \\
2 & 0.259 & 0.023 & -0.017 & 318 \\
3 & 0.5 & 0.0 & 0.487 & 157 \\
6 & 0.236 & 0.259 & 0.104 & 198 \\
7 & 0.518 & 0.289 & 0.135 & 258 \\
10 & 0.229 & 0.518 & 0.002 & 318 \\
\hline
\end{tabular}

cf. $u, v, w$; fractional coordinates ( see Fig.5-4).

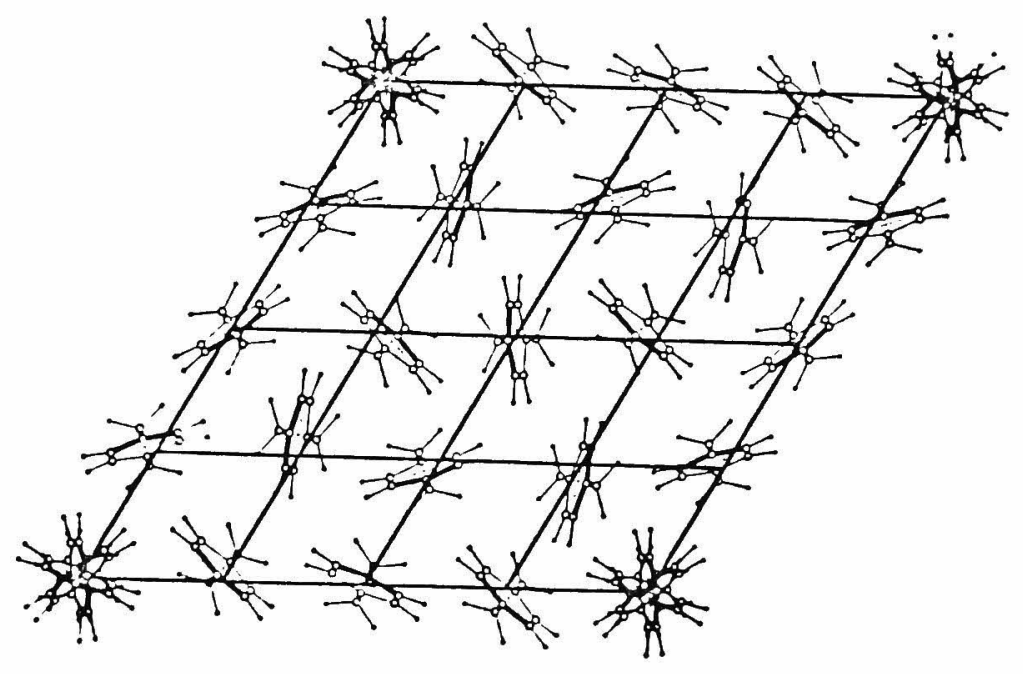

Fig.5-10 Three-dimensional structure corresponding to Table 5-5(a).

Bold lines of a molecule represent the upper part of a benzene ring. 
Table 5-5(b)

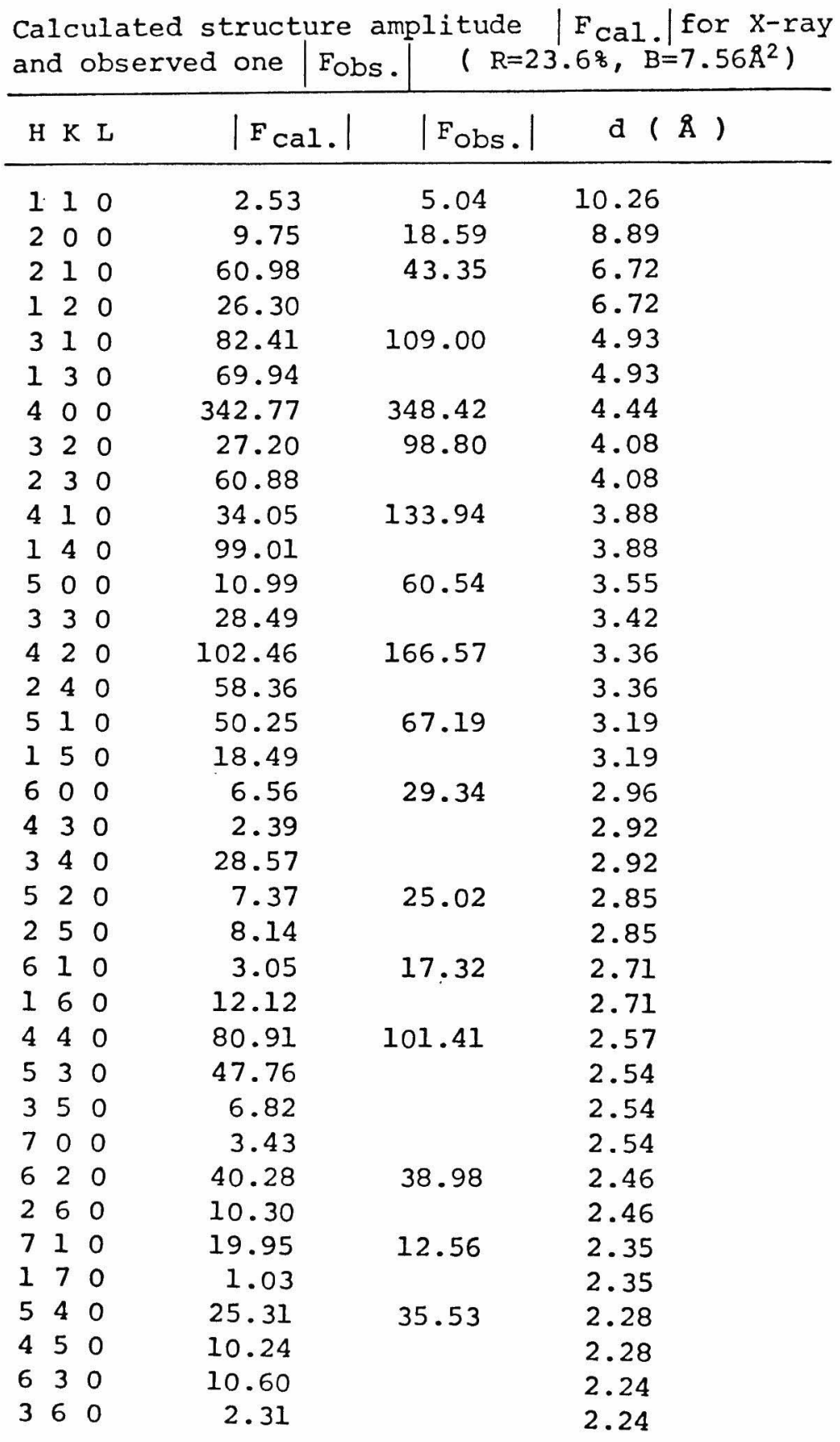


Table 5-5(b) ( continued )

\begin{tabular}{rrrrrr}
\hline$H$ & $K$ & $L$ & $\left|F_{\text {Cal }}\right|$ & $\left|F_{\text {obs }}\right|$ & $d(\AA)$ \\
\hline 2 & 0 & 1 & 60.46 & 65.84 & 5.29 \\
0 & 2 & 1 & 21.23 & & 5.29 \\
2 & 1 & 1 & 14.93 & 9.70 & 4.70 \\
1 & 2 & 1 & 25.66 & & 4.70 \\
-2 & 3 & 1 & 11.68 & & 4.70 \\
-1 & 3 & 1 & 15.92 & & 4.70 \\
3 & 1 & 1 & 12.20 & 41.50 & 3.95 \\
1 & 3 & 1 & 9.79 & & 3.95 \\
-3 & 4 & 1 & 48.06 & & 3.95 \\
-1 & 4 & 1 & 21.94 & & 3.95 \\
3 & 2 & 1 & 15.55 & & 3.47 \\
2 & 3 & 1 & 20.01 & & 3.47 \\
-3 & 5 & 1 & 31.75 & & 3.47 \\
-2 & 5 & 1 & 34.51 & & 3.47 \\
4 & 0 & 1 & 11.36 & 75.19 & 3.68 \\
0 & 4 & 1 & 24.58 & & 3.68 \\
4 & 1 & 1 & 20.14 & 55.87 & 3.34 \\
1 & 4 & 1 & 8.72 & & 3.34 \\
-4 & 5 & 1 & 11.84 & & 3.34 \\
-1 & 5 & 1 & 68.81 & & 3.34 \\
4 & 2 & 1 & 118.42 & 181.54 & 2.99 \\
2 & 4 & 1 & 38.07 & & 2.99 \\
-4 & 6 & 1 & 61.92 & & 2.99 \\
-2 & 6 & 1 & 60.01 & & 2.99 \\
3 & 3 & 1 & 80.60 & & 3.03 \\
-3 & 6 & 1 & 30.95 & & 3.03 \\
5 & 1 & 1 & 34.76 & 30.89 & 2.87 \\
1 & 5 & 1 & 54.53 & & 2.87 \\
-5 & 6 & 1 & 38.47 & & 2.87 \\
-1 & 6 & 1 & 50.21 & & 2.87 \\
6 & 0 & 1 & 27.04 & 40.04 & 2.70 \\
0 & 6 & 1 & 3.93 & & 2.70 \\
4 & 3 & 1 & 20.33 & & 2.67 \\
3 & 4 & 1 & 27.08 & & 2.67 \\
-4 & 7 & 1 & 25.59 & & 2.67 \\
-3 & 7 & 1 & 53.49 & & \\
& & & & \\
\hline
\end{tabular}


Table 5-5(b) (continued)

\begin{tabular}{|c|c|c|c|c|c|}
\hline $\mathrm{H}$ & $\mathrm{K}$ & $L$ & $F_{\text {cal. }} \mid$ & $\left|F_{\text {obs. }}\right|$ & $d(\AA)$ \\
\hline 5 & 2 & 1 & 40.60 & 40.45 & 2.61 \\
\hline 2 & 5 & 1 & 32.78 & & 2.61 \\
\hline-5 & 7 & 1 & 43.68 & & 2.61 \\
\hline-2 & 7 & 1 & 20.01 & & 2.61 \\
\hline 6 & 1 & 1 & 14.86 & & 2.51 \\
\hline$I$ & 6 & 1 & 36.28 & & 2.51 \\
\hline-6 & 7 & 1 & 56.63 & & 2.51 \\
\hline-1 & 7 & 1 & 40.45 & & 2.51 \\
\hline 5 & 3 & 1 & 17.86 & 90.43 & 2.37 \\
\hline 3 & 5 & 1 & 62.33 & & 2.37 \\
\hline-5 & 8 & 1 & 37.52 & & 2.37 \\
\hline-3 & 8 & 1 & 34.26 & & 2.37 \\
\hline 0 & 7 & 1 & 21.79 & & 2.37 \\
\hline 7 & 0 & 1 & 69.46 & & 2.37 \\
\hline 5 & 4 & 1 & 16.49 & 52.40 & 2.15 \\
\hline 4 & 5 & 1 & 22.75 & & 2.15 \\
\hline-5 & 9 & 1 & 64.62 & & 2.15 \\
\hline-4 & 9 & 1 & 19.15 & & 2.15 \\
\hline 4 & 2 & 2 & 8.41 & 34.67 & 2.35 \\
\hline 2 & 4 & 2 & 7.67 & & 2.35 \\
\hline-4 & 6 & 2 & 9.54 & & 2.35 \\
\hline-2 & 6 & 2 & 17.30 & & 2.35 \\
\hline 5 & 1 & 2 & 9.94 & & 2.29 \\
\hline 1 & 5 & 2 & 14.24 & & 2.29 \\
\hline-5 & 6 & 2 & 1.82 & & 2.29 \\
\hline-1 & 6 & 2 & 11.46 & & 2.29 \\
\hline 6 & 0 & 2 & 7.90 & 34.13 & 2.20 \\
\hline 0 & 6 & 2 & 23.99 & & 2.20 \\
\hline 4 & 3 & 2 & 8.03 & & 2.18 \\
\hline 3 & 4 & 2 & 6.42 & & 2.18 \\
\hline-4 & 7 & 2 & 11.18 & & 2.18 \\
\hline-3 & 7 & 2 & 15.07 & & 2.18 \\
\hline 5 & 3 & 2 & 19.69 & 100.09 & 2.01 \\
\hline 3 & 5 & 2 & 9.30 & & 2.01 \\
\hline-5 & 8 & 2 & 24.37 & & 2.01 \\
\hline-3 & 8 & 2 & 13.45 & & 2.01 \\
\hline 7 & 0 & 2 & 7.37 & & 2.01 \\
\hline 0 & 7 & 2 & 3.77 & & 2.01 \\
\hline 4 & 4 & 2 & 20.36 & & 2.02 \\
\hline-4 & 8 & 2 & 31.37 & & 2.02 \\
\hline
\end{tabular}




\begin{tabular}{|c|c|c|c|c|c|}
\hline $\mathrm{H}$ & $\mathrm{K}$ & L & $F_{\mathrm{cal}}$. & I $F_{\text {obs. }}$ & $\mathrm{d}(\AA)$ \\
\hline 6 & 2 & 2 & 37.48 & 55.24 & 1.97 \\
\hline 2 & 6 & 2 & 17.03 & & 1.97 \\
\hline-6 & 8 & 2 & 13.53 & & 1.97 \\
\hline-2 & 8 & 2 & 26.11 & & 1.97 \\
\hline 7 & 1 & 2 & 13.18 & & 1.91 \\
\hline 1 & 7 & 2 & 21.42 & & 1.91 \\
\hline-7 & 8 & 2 & 9.95 & & 1.91 \\
\hline-1 & 8 & 2 & 26.84 & & 1.91 \\
\hline 5 & 4 & 2 & 10.71 & 77.81 & 1.87 \\
\hline 4 & 5 & 2 & 11.12 & & 1.87 \\
\hline-5 & 9 & 2 & 15.23 & & 1.87 \\
\hline-4 & 9 & 2 & 12.27 & & 1.87 \\
\hline 6 & 3 & 2 & 26.49 & & 1.85 \\
\hline 3 & 6 & 2 & 16.70 & & 1.85 \\
\hline-6 & 9 & 2 & 11.47 & & 1.85 \\
\hline-3 & 9 & 2 & 31.13 & & 1.85 \\
\hline 8 & 0 & 2 & 13.76 & & 1.84 \\
\hline 0 & 8 & 2 & 40.59 & & 1.84 \\
\hline 4 & 0 & 3 & 23.03 & 96.06 & 1.97 \\
\hline 0 & 4 & 3 & 32.74 & & 1.97 \\
\hline 3 & 2 & 3 & 34.39 & & 1.93 \\
\hline 2 & 3 & 3 & 30.05 & & 1.93 \\
\hline-3 & 5 & 3 & 38.11 & & 1.93 \\
\hline-2 & 5 & 3 & 45.92 & & 1.93 \\
\hline 4 & 2 & 3 & 28.41 & 70.59 & 1.84 \\
\hline 2 & 4 & 3 & 37.59 & & 1.84 \\
\hline-4 & 6 & 3 & 22.49 & & 1.84 \\
\hline-2 & 6 & 3 & 4.04 & & 1.84 \\
\hline 5 & 1 & 3 & 19.71 & & 1.81 \\
\hline 1 & 5 & 3 & 16.55 & & 1.81 \\
\hline-5 & 6 & 3 & 19.87 & & 1.81 \\
\hline-1 & 6 & 3 & 26.80 & & 1.81 \\
\hline 5 & 3 & 3 & 18.81 & 46.93 & 1.66 \\
\hline 3 & 5 & 3 & 22.26 & & 1.66 \\
\hline-5 & 8 & 3 & 15.74 & & 1.66 \\
\hline-3 & 8 & 3 & 13.15 & & 1.66 \\
\hline 7 & 0 & 3 & 11.18 & & 1.66 \\
\hline 0 & 7 & 3 & 16.22 & & 1.66 \\
\hline 4 & 4 & 3 & 22.49 & & 1.67 \\
\hline-4 & 8 & 3 & 3.60 & & 1.67 \\
\hline
\end{tabular}




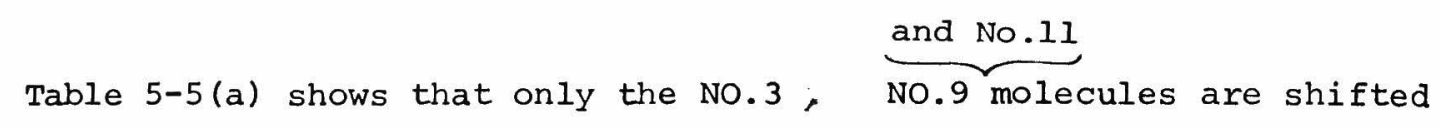
by about half of the period along the c-axis, and other molecules locate at almost the same level.

Here we have reached the stage where the consistency of the result of the $\mathrm{X}$-ray analysis and the high resolution electron micrograph should be examined. Kobayashi says that seeing is not always believing, near the focus of a microscope at its resolution limit. The image intensity is greatly affected by the defocus and the spherical aberration through $\sin \chi(u)$ in phase contrast electron microscopy, as shown in Eq.(1-18) in Chapter 1. Therefore it is necessary for us to compare the obtained electron micrograph with simulated through-focal images, so as to confirm authenticity of the micrograph as well as the result of structure analysis. Thus we simulated, with an electronic computer, the through-focusing images to be taken with JEM-500 ( accelerating voltage, 500KV; spherical aberration coefficient, $C_{s}=1.06 \mathrm{~mm}$, resolving power, $\left.1.4 \AA\right)$. In the calculation, an imaginary crystal with the final structure parameters (Table 5-5(a)) was assumed. The computer simulation was performed according to the kinematical imaging theory ( see Chapter 1), and the results were displayed with the facsimile receiver ( see Chapter 4). The result is shown in Fig.5-11. This computer-simulation confirms that the obtained micrograph (Fig.5-2) is nearly the best one that can be expected from JEM-500. Therefore, we can not expect to resolve up to the individual carbon 
atoms of a PPX molecule in the ab-plane projection in which atoms are close together.

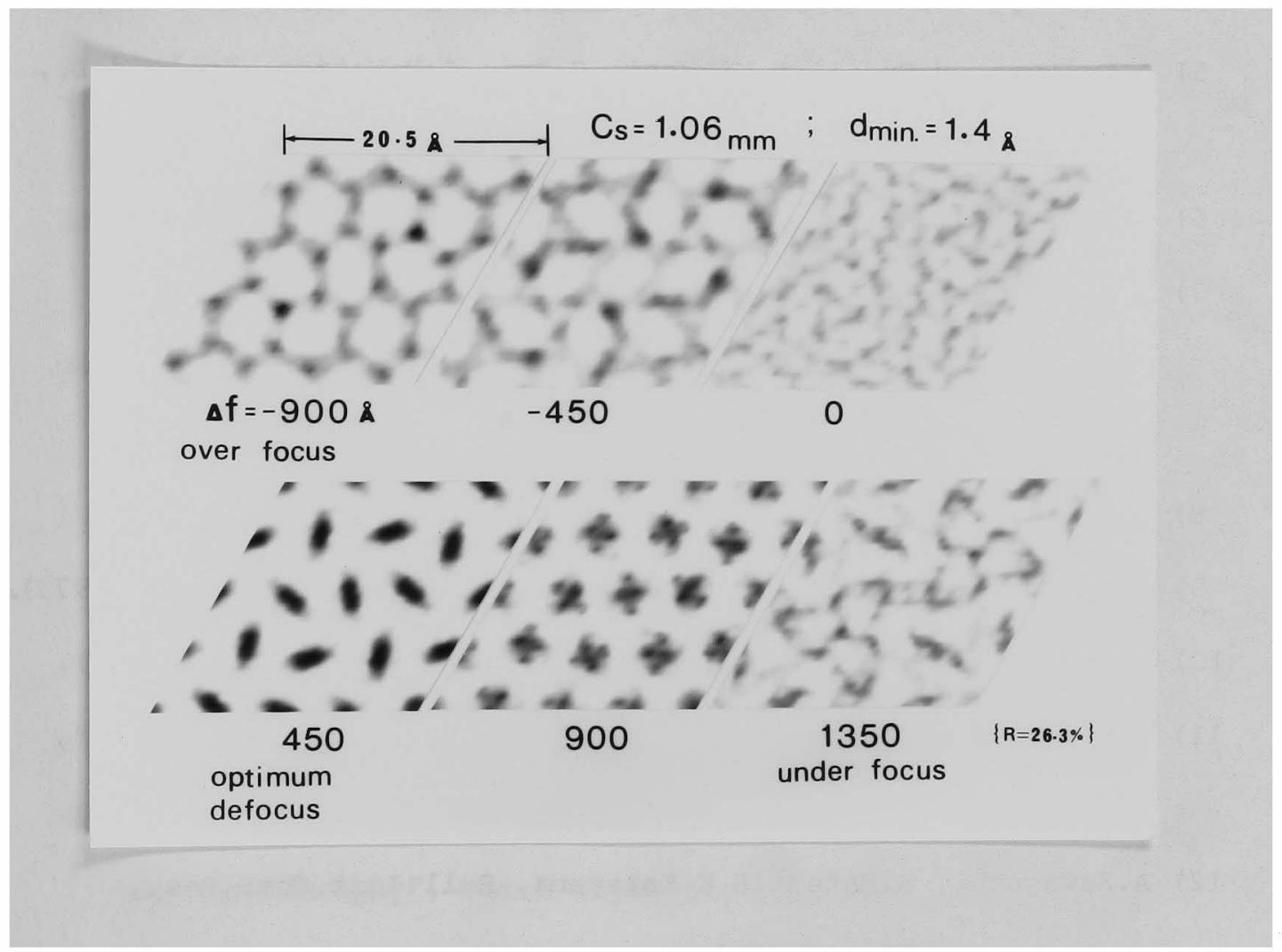

Fig.5-11 Simulated through-focal images to be taken with JEM-500 ( wave length; $0.0142 \AA$ ).

$\Delta f$; amount of defocusing (positive for under-focus). 
References

1) C.J.Brown \& A.C.Farthing, J.Chem.Soc., 1953, 3270 (1953).

2) M.H.Kaufman, H.F.Mark \& R.B.Mesrobian, J.Polymer Sci., 13, $3(1954)$

3) W.D.Niegisch, J.Appl.Phys., 37, 4041 (1966).

4) W.D.Niegisch, J.Polymer Sci., B-4, 531 (1966).

5) A.Kajiura, M.Fujii, K.Kikuchi, S.Irie \& H.Watase, Kolloid-Z., $\underline{224}, 124(1968)$.

6) S.Kubo \& B.Wunderlich, J.Appl.Phys., 42, 4565(1971).

7) L.Lieser, Thesis, Naturwissenschaftliche Fakultat der Johannes-Gutenberg-Universität zu Mainz, Mainz, (1971); cited in Ref.(8).

8) S.Kubo \& B.Wunderlich, Makromol.Chem., 162, 1(1972).

9) R.Iwamoto \& B.Wunderlich, J.Polymer Sci.-Phys., 11, 2403 (1973).

10) M.Miles \& H.Gleiter, J.Macromol.Sci.-Phys., B-15, 613(1978).

11) B.K.Vainshtein, "Structure Anlysis by Electron Diffraction", Pergamon-Press, Chapter 3, (1964).

12) A.Kawaguchi, R.Matsui \& K.Katayama, Bull.Inst.Chem.Res., Kyoto Univ., 58, $470(1980)$.

13) R.J.Samuels \& R.Y.Yee, J.Polymer Sci.:A-2, 10, 385(1972).

14) T.Uchida \& H.Tadokoro, ibid., 5, 63(1967).

15) G.Natta \& P.Corradini, Makromol.Chem., 16, 77(1955).

16) G.Natta, Angew.Chem., 68, 393(1956). 
17) S.Sasaki, Y.Takahashi \& H.Tadokoro, Discuss.Meet.Soc.Polym.Sci.Japan, Osaka, Preprint, 491 (1972).

18) P.Corradini \& I.W.Bassi, J.Polymer Sci.:C, 16, 3233(1968).

19) C.A.Sperati \& H.W.Starkweather JR, Adv.Polymer Sci., $\underline{2}$, 465 (1961).

20) Z.Mencik, J.Polymer Sci.-Phys., 11, 1585 (1973).

21) Y.Kinoshita, Makromol.Chem., 33, 21 (1959).

22) H.Tadokoro, Y.Takahashi, Y.Chatani \& H.Kakida, Makromol.Chem., $109,96(1967)$.

23) H.Tadokoro, "Structure of Crystalline Polymers", John Wiley \& Sons, Chapter 7, (1979).

24) L.E.Alexander, "X-ray Diffraction Methods in Polymer Science", John Wiley \& Sons, Appendix 3, (1969).

25) R.L.Miller, in "Polymer Handbook", 2-nd ed., Ed. by J.Brandrup \& E.H.Immergut, John Wiley \& Sons, (1975).

26) W.D.Niegisch, J.Appl.Phys., 38, 4110 (1967).

27) S.Kubo \& B.Wunderlich, J.Polymer Sci.-Phys., 10, 1949 (1972).

28) N.F.M.Henry \& K.Lonsdale Ed., "International Tables for X-ray Crystallography", vol.1 (Symmetry Groups), Kynoch, (1965).

29) A.Kawaguchi, Thesis, Kyoto University, Chapter 2, (1979).

30) K.Kobayashi, Proc.EMAG-75, Bristol, 251 (1976). 
Chapter 6 : Direct observation of the lattice defects in $\mathrm{PPX}$ single crystals

High resolution electron microscopy has developed to such an iff extent that details on a molecular or atomic scale can be observed and the information on atomic positions be obtained directly. In : itc contrast to microscopy, diffraction experiments (x-rays, neutrons, defe electrons) give only statistical data averaged over the whole wnt sample. The electron microscope (EM) is therefore a powerful and inar unique tool with which to study defects in the periodic structure of the a crystal visually.

Polymer crystals are much more imperfect than crystals of sinc ordinary low-molecular-weight materials, as shown by $\mathrm{X}$-ray diffraction. Based on the electron micrographs with moiré-image 3-7

interference fringes ( usually explained in terms of double Bragg

diffraction of the electron beam) or dislocation networks 
(observed by virtue of diffraction contrast) in multi-layered polymer single crystals, the imperfections in polymer crystals have been made considerably clear. Lattice defects in a polyethylene (PE) single crystal due to electron irradiation were also investigated by the moiré method. It was reported that the image intensity of the moiré fringe patterns in electron micrographs of two superposed thin crystals is "the square of the Patterson distribution", magnified by the factor $1 / 2 \sin (\varepsilon / 2)(\varepsilon$ is the relative rotation of the crystals ). Therefore it is difficult to analyse the structure of dislocation on a molecular or atomic scale. Moreover, the analysis of dislocation networks is much more difficult than that of moiré fringes.

In the cases of inorganic or organic crystals which are very strong against electron irradiation, high resolution images of defects and dislocations can be obtained. Polymer crystals, on the contrary, are so weak against electron irradiation that the lattice images including the defects have never been obtained. We have for the first time succeeded in obtaining a high resolution lattice image which resolves directly the edge dislocation in a PPX $\beta$-form single crystal, as described in Chapter 4. In this chapter, this image is considered in the light of dislocation models. 
6-1 Models of dislocation in a polymer crystal

Since the constituent atoms in a polymer crystal are linked together with covalent bonds to form molecules, the direction of the backbone chain is a unique direction in the crystal. The classification of the dislocations in PE orthorhombic crystal was first carried out by Keith \& Passaglia. Seto classified dislocations in polymer crystals into 5 types ( Table 6-1) on the basis of the directional relationship among the Burgers vector $\mathbf{b}$, the vector of the backbone chain direction $\mathbf{m}$, and the vector of the dislocation-line direction I. Figure 6-1 schematically shows dislocation models corresponding to the 5 types in Table 6-1. Wada \& Matsui ${ }^{16}$ reclassified the dislocations in PE in terms of these 5 types. The characteristics of the dislocation types shown in Table 6-1 and Fig.6-1 are summarized as follows:

Type (1) : This is the screw dislocation that does not change the conformation of polymer chains. This can be first produced at the side face of a thin polymer single crystal owing to thermal motion, and then proceeds into the crystal.

Type (2) : This edge dislocation was proposed in order to account for the slip bands in plastic deformation of Nylon $66^{18}$ "This dislocation is considered to be much less likely because of the registance to glide motion, especially in polymers where the 
Table 6-1

Dislocations in Polymer Crystals

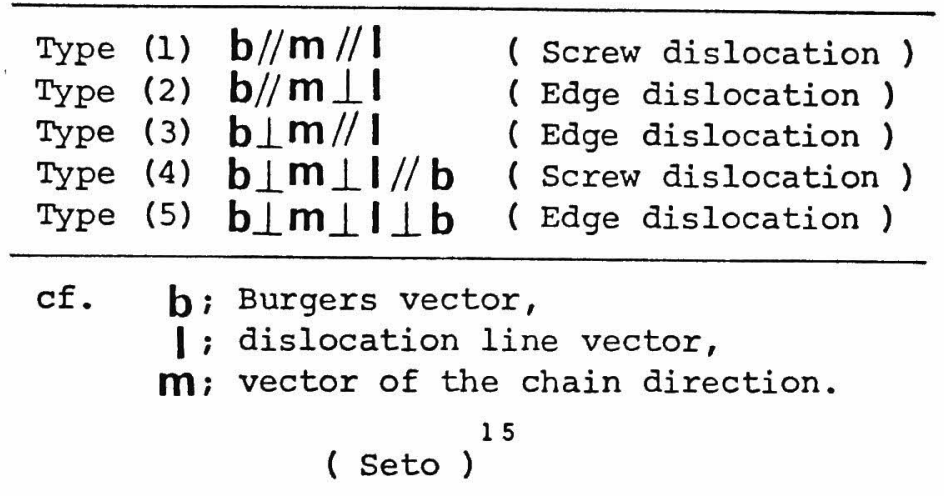
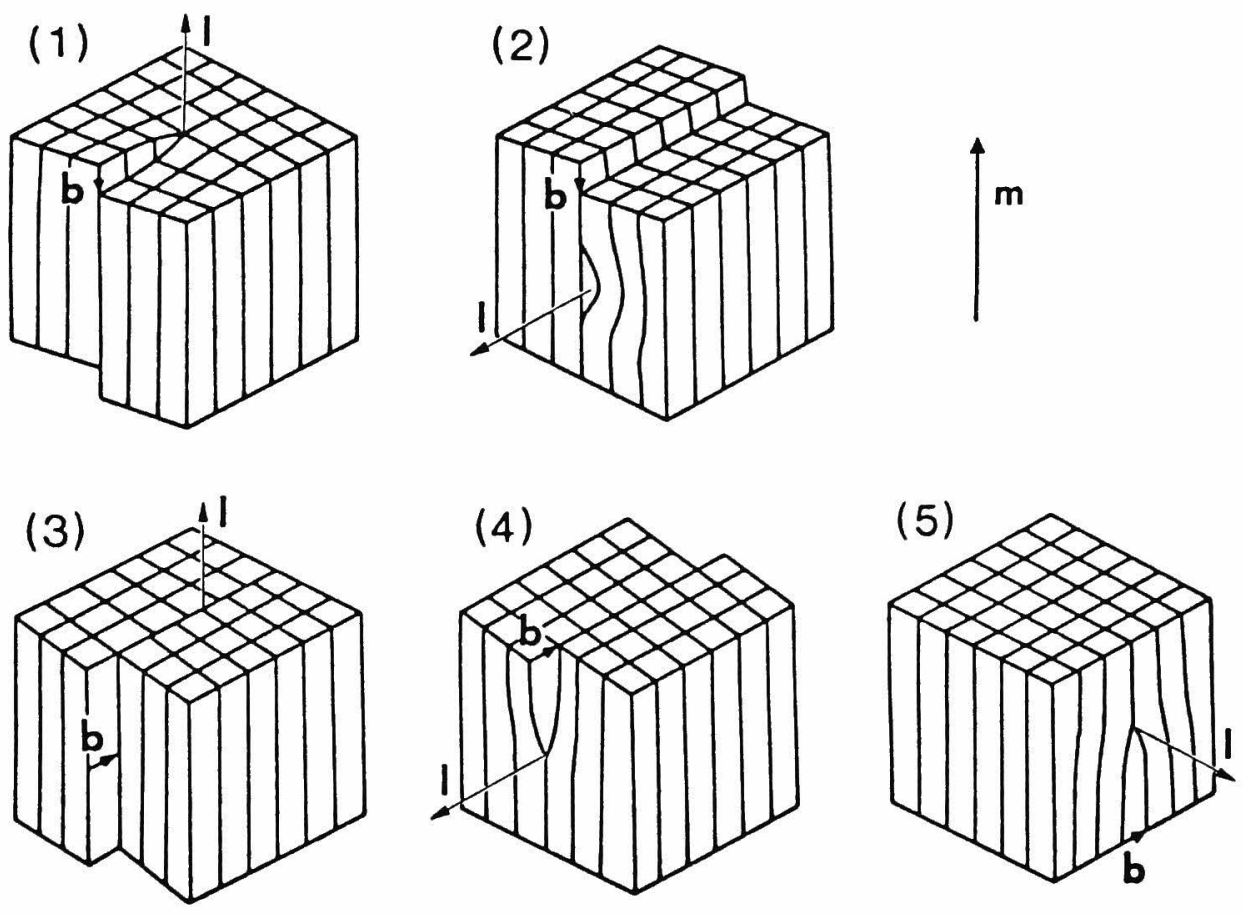

Fig.6-1 Models of dislocations in polymer crystals corresponding to Table 6-1.

A square prism corresponds to a molecular chain.

$$
(\text { Seto })^{15}
$$


chemical repeat distance is very long.

Type (3) : This dislocation is most stable because this does not h change the conformation of the polymer chains and passes through a di lamellar crystal in the shortest course. This was observed through moiré fringes in lamellar crystals ( such as polyoxymethylene, 5 and so on ) with EM.

Type (4) : This dislocation is energitically unstable. A concentration of dislocations of this type generates a twist boundary 19

in a crystal. Hosemann et al. cosidered that the polymer single crystals are mosaic crystals from the line profile analysis of on (hho) $\mathrm{x}$-ray reflections, and ascribed the mosaic nature of polymer dos cystals to this type of dislocation.

Type (5) : This type of dislocation is generated when the ends of res chains stand in a line. A tilt boundary is caused by a anc concentration of dislocations of this type 20

20

The screw dislocation of Type (1) which has the Burgers vector agi parallel to the molecular axis and the edge dislocation of Type (3) whose Burgers vector is perpendicular to the molecular axis, should wit be very easily introduced in lamellar crystals. When the molecular dis axis in the thin lamellar crystal is perpendicular to the crystal pk end-surface, it seems practically difficult to observe the dislocations of Types (1) and (2) because their Burgers vectors are it parallel to incident electrons. In the case of Types (3), (4) and in 
(5) dislocations can be observed through EM. The dislocation which has been observed in a PE single crystal corresponds to the partial dislocation of Type $(3)^{7,2}$.

6-2 High resolution electron microscopy for observing dislocations in PPX single crystals

The edge dislocations in inorganic crystals have been observed $22-25$
on an atomic scale, and the point defects have also been directly 25-28

observed, by high resolution electron microscopy. A high-angle tilt grain boundary in a thin crystal of germanium was imaged with atomic resolution and was shown to consist of alternating columns of fiveand seven-membered rings of germanium atoms. The dislocations of various types are observed in the crystal of organic compounds such as hexadeca-chlorocopper phthalocyanine when the specimen is strong against electron irradiation.

In order to obtain high resolution images of crystal defects with an electron microscope, the defects should extend along the direction of incident electrons, forming columns which produce proper contrast in an image.

The edge dislocation image of a PPX $B$-form single crystal in Fig.4-10(c) is recited in Fig.6-2, which is the optically filtered image. The arrangement of molecules in the projection of the 
ab-plane is not very clear. However, the appearance of the dislocation shown in Fig.6-2 is similar to Bragg \& Nye's bubble 30

model of the edge dislocation, which is shown in Fig.6-3. Either $O^{\prime} X^{\prime}$ or $O^{\prime} Y^{\prime}$ in Fig.6-3 is possibly an additional extra plane. Similarly, either OX or OY in Fig.6-2 should be an extra plane. The obtained dislocation should be the partial one of Type (3), with the magnitude of the Burgers vector being one fourth of the lattice constant $(a=20.52 \AA$, see Chapter 5). Neither the mutual arrangement nor orientations of molecular chains are able to be defined in Fig.6-2, owing to lack of higher resolution. Thus stacking faults associated with this partial dislocation are not confirmed.

We were also able to obtain high resolution (020) lattice images including lattice defects, of a PPX $\alpha$-form single crystal ( Fig.6-4; Fig.4-9(c) recited, which was optically processed). Figure 6-4 suggests that the additional plane is parallel to (010) 


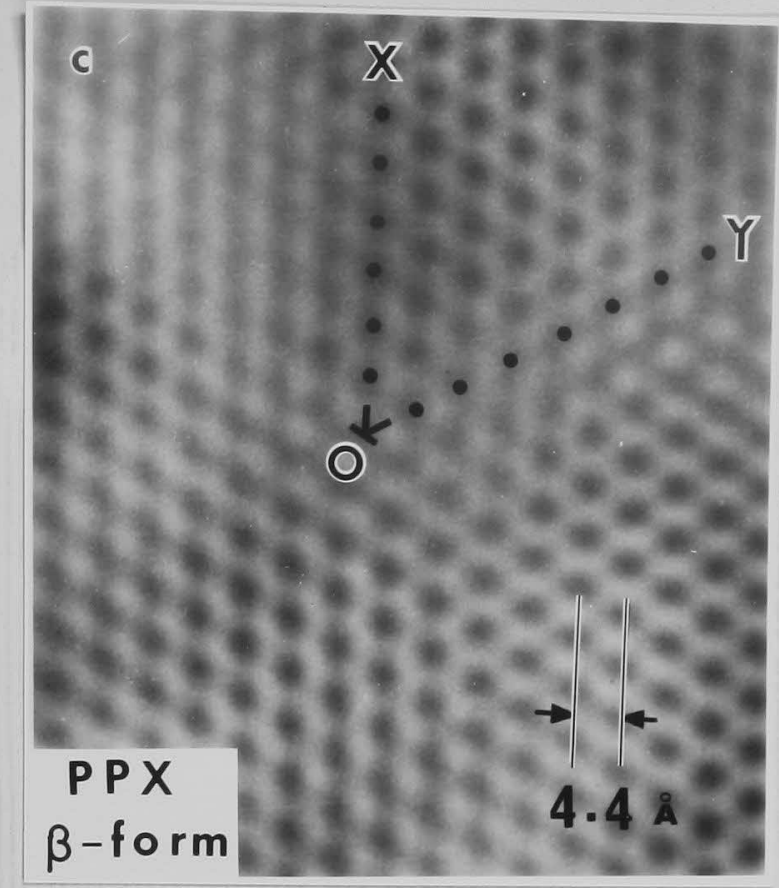

Fig.6-2 Edge dislocation in a PPX $\beta$-form single crystal.

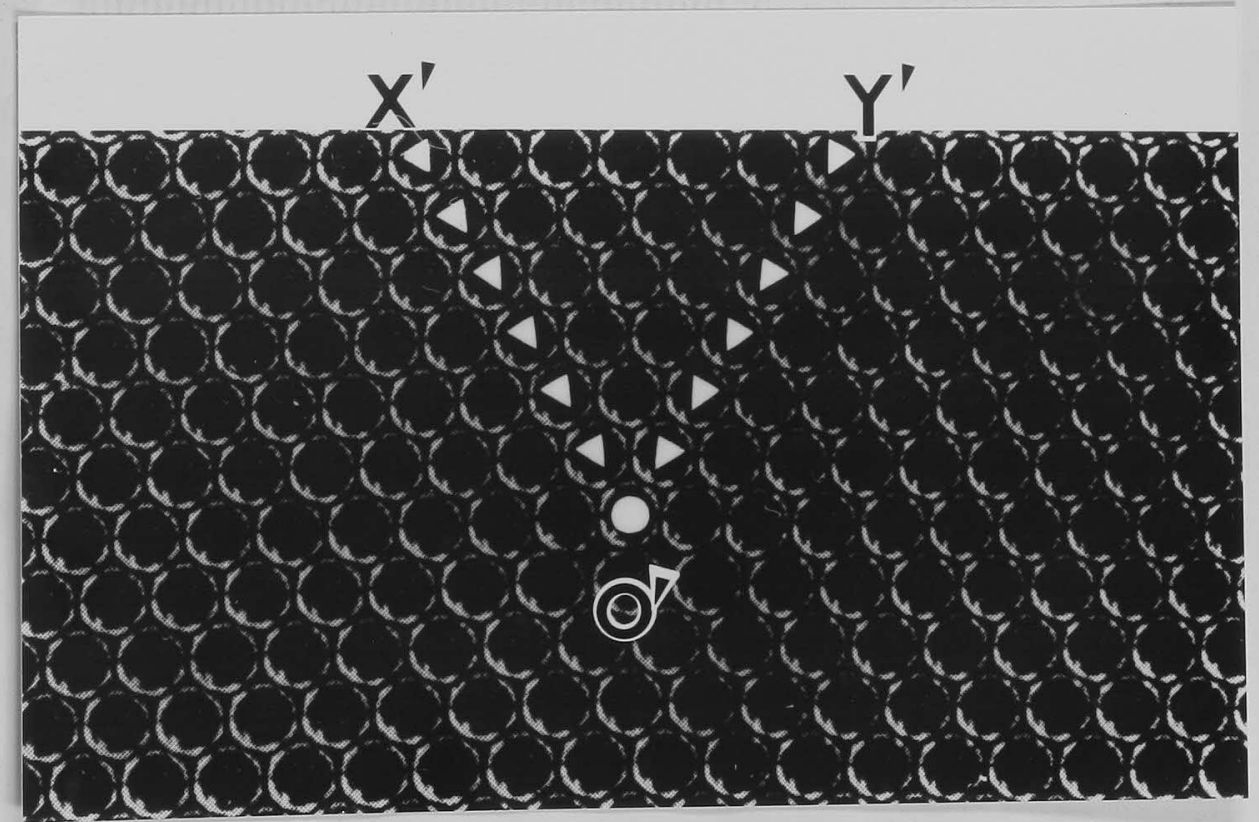

Fig.6-3 Edge dislocation in the bubble-raft.

$$
\text { ( Bragg \& Nye })^{30}
$$




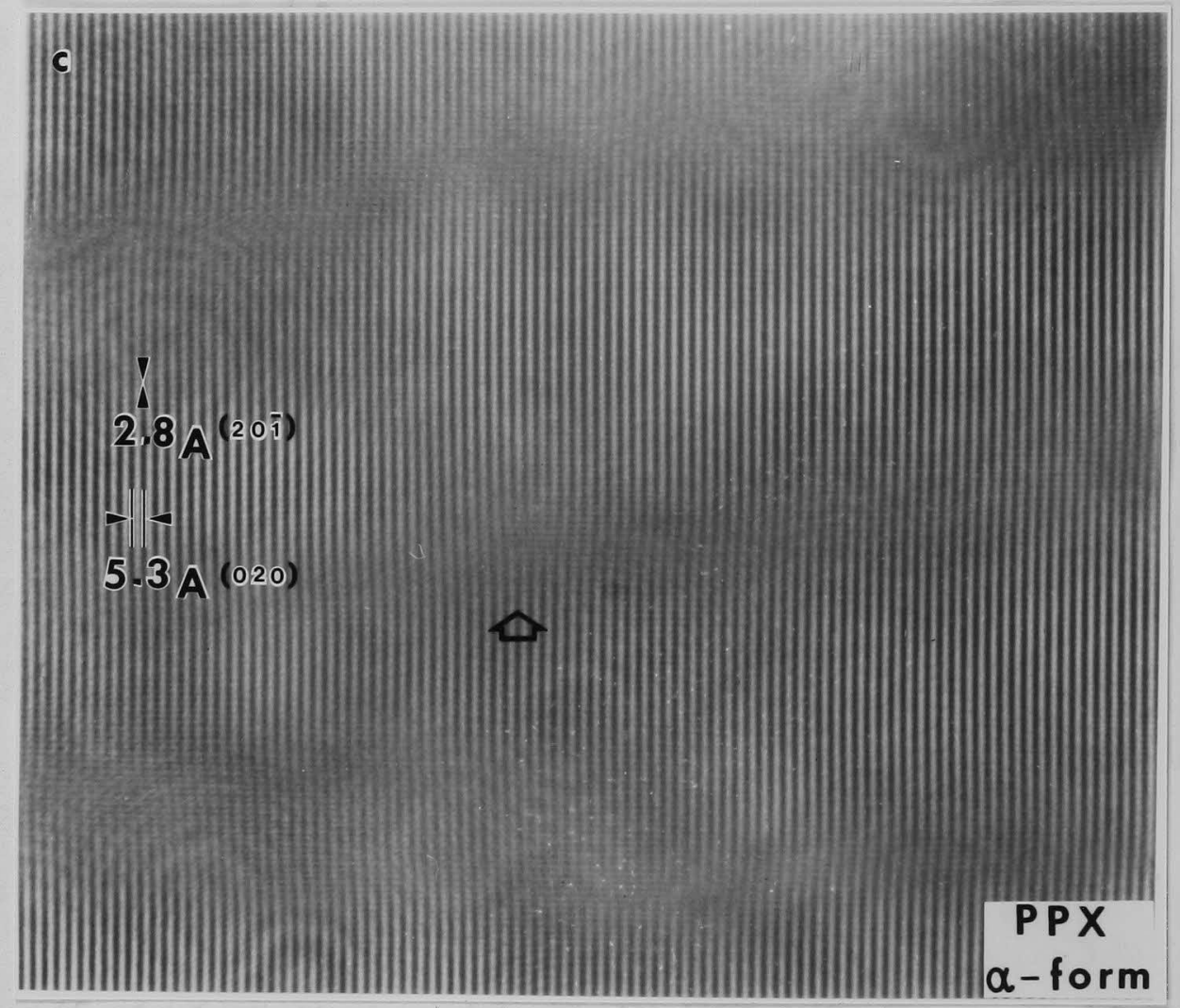

Fig.6-4 Edge dislocation in a PPX $\alpha$-form single crystal. An arrow shows the position of a dislocation. 
References

1) S.Iijima \& J.G.Allpress, Acta Cryst., A $\underline{30}, 22(1974)$.

2) A.J.Skarnulis, S.Iijima \& J.M.Cowley, Acta Cryst., А32, $799(1976)$

3) A.W.Agar, F.C.Frank \& A.Keller, Phil.Mag., 4 , 32(1959).

4) V.F.Holland, J.Appl.Phys., 35, 1351 (1964).

5) D.C.Bassett, Phil.Mag., 10, 595 (1964).

6) V.F.Holland, J.Appl.Phys., 35, 3235 (1964).

7) P.H.Lindenmeyer, J.Polymer Sci., C-15, 109 (1966).

8) V.F.Holland \& P.H.Lindenmeyer, Science, 147, 1296 (1965).

9) V.F.Holland, P.H.Lindenmeyer, R.Trivedi \& S.Amelinckx, Phys.stat.Sol., 10, $543(1965)$.

10) V.F.Holland \& P.H.Lindenmeyer, J.Appl.Phys., 10, 3049 (1965).

11) T.Nagasawa \& K.Kobayashi, J.Appl.Phys., 4l , 4276 (1970).

12) W.C.T.Dowell, J.L.Farrant \& A.L.G.Rees, Proc.4-th Int.Conf.EM, (Berlin 1958), Springer-Verlag, 367(1960).

13) Y.Murata, T.Baird \& J.R.Freyer, Nature, 262, 721 (1976).

14) H.D.Keith \& E.Passaglia, J.Res.NBS, $\underline{\text { A-68, }} 513(1964)$.

15) T.Seto, Zairyo-kagaku, 4, 178 (1967).

16) Y.Wada \& M.Matsui, Kobunshi(High Polyms.Japan), 19, 658(1970).

17) J.M.Peterson, J.Appl.Phys., 37, 4047 (1966) .

18) D.A.Zaukelis, J.Appl.Phys., 33, 2797(1962). 
19) R.Hosemann, W.Wilke \& F.J.Balta Calleja, Acta Cryst., 21, $118(1966)$.

20) P.Predecki \& W.O.Statton, J.Appl.Phys., 37, 4053(1966).

21) G.A.Bassett, J.W.Menter \& D.W.Pashley, Proc.Roy.Soc.London, $\underline{A-246}, 345$ (1958).

22) A.Bourret, J.Desseaux \& A.Renault, J.Microsc.Spectosc.Electron, 2, 467 (1977) .

23) A.Bourret \& J.Desseaux, Nature, 272, 151 (1978).

24) A.Bourret \& J.Desseaux, Phil.Mag., A-39, 405 (1979).

25) A.Bourret \& J.Desseaux, ibid., $\underline{A-39}, 419$ (1979).

26) S.Iijima, Acta Cryst., A-29, 18(1973).

27) S.Iijima, S.Kimura \& M.Goto, Acta Cryst., A-29, 632(1973).

28) S.Iijima, S.Kimura \& M.Goto, ibid., $\underline{A-30}, 251$ (1974).

29) O.L.Krivanek, S.Isoda \& K.Kobayashi, Phil.Mag., 36, 931 (1977).

30) W.L.Bragg \& J.F.Nye, Proc.Roy.Soc., A-190, 474 (1947). 
Summary

Chapter 1 : Some aspects of high resolution electron microscopy

Poly (p-xylylene) [PPX] crystals are about 10 times as strong as $\mathrm{PE}$ crystals against electron irradiation. The chain direction in a thin single crystal of the PPX $B$-form is perpendicular to the crystal end-surface. Thus the individual chains composing this crystal are expected to be resolved.

Scattering of electrons through such a weak phase object and image formation in high resolution electron microscopy are outlined, followed by some practical problems in taking high resolution electron micrographs of PPX single crystals with JEM-500. 
Chapter 2 : Morphology of PPX single crystals

Morphology of both $\alpha$ - and $\beta$-form PPX single crystals, which were to be adopted as specimens for high resolution electron microscopy, was discussed. Two entirely different types of single crystals developed from dilute solution. A rectangular crystal is the $\alpha$-form and a hexagonal crystal the $\beta$-form.

In the case of multi-layered $\alpha$-form crystals, it was confirmed that the chain axis in the basal single layer is not perpendicular to the support-film, but the chain axis in other layers is perpendicular to it. Moiré patterns were observed in the multi-layered crystal of the $\alpha$-form.

We have confirmed that the electron diffraction pattern of the B-form single crystal shows no systematic absences of reflections and has no symmetry planes, but shows only the 6-fold rotational symmetry. Twin-like crystals and spirally grown crystals of the $\beta$-form were observed, but no moiré fringes were observed.

At the end of this chapter, morphological changes of both $\alpha$ and $\beta$-form single crystals due to electron irradiation were also discussed. 
Chapter 3 : High resolution electron microscopy

of PPX single crystals

Changes of lattice spacings of PPX $\alpha$ - and $\beta$-form single crystals due to $500 \mathrm{KV}$-electron irradiation were measured, in order to estimate the radiation-resistivity of PPX crystals for $500 \mathrm{KV}$ electrons. The total end point dose (TEPD), namely the electron dose necessary for complete damage of a PPX single crystal was about 0.4 Coulombs $/ \mathrm{cm}^{2}$ (on the specimen).

Based on this result, the conditions for taking high resoiution electron micrographs of PPX single crystals, such as magnification and so on, were established. We have attempted to resolve individual chains of PPX with JEM-500, and finally have succeeded in taking high resolution electron micrographs of both the PPX $\alpha$ - and $\beta$-form single crystals. Especially the individual chains composing a $\beta$-form single crystal were able to be observed in the projection on the ab-plane.

Chapter 4 : Image processing for electron microscopy

The similarity between the electron diffraction pattern of a PPX $B$-form single crystal and the optical diffractogram of its high resolution micrograph confirms that the micrograph sufficiently 
reflects the crystal structure of the $\beta$-form, though the details expected from the diffractogram were obscured by the low $\mathrm{S} / \mathrm{N}$ ratio due to the photographic graininess. In order to extract the desired information from such a micrograph, random noises due to graininess should be eliminated by image processing.

In this chapter, first we described the construction of image processing systems and also discussed the image averaging by spatial frequency filtering. Especially the effect of the pinhole size of a filter grating in optical filtering was theoretically discussed.

The high resolution micrograph of a $P P X \quad \beta$-form single crystal was thus processed optically with our system and the periodical structure in the micrograph was effectively accentuated. This processed image showed mutual positions of the chains in the crystal in the ab-plane projection. Moreover, micrographs with lattice defects were also processed using proper filters to improve the $\mathrm{S} / \mathrm{N}$ ratio.

Chapter 5 : Crystal structure analysis of PPX B-form crystal

As regards the PPX $\beta$-form crystal, the detailed crystal structure is not known. It is concluded that a unit cell (hexagonal or trigonal) of the $\beta$-form crystal contains 16 chain-segments ( the segmental length is the fiber period, , from its observed density 
and lattice constants. This fact made it difficult to analyse the crystal structure of the $\beta$-form.

The optically filtered image of a PPX $\beta$-form single crystal indicates the mutual arrangement of chains in the ab-plane projection and gives the starting point of crystal structure analysis. We first assumed a 2-dimensional structure model (space group : p6 ), taking van der Waals' radii, the electron microscopic image, and the 6-fold rotational symmetry in the electron diffraction pattern into account. The orientation of the chain at the origin of a unit cell was assigned statistically to one of three equivalent orientations to satisfy the symmetry of p6. The refinement of the structure analysis was performed by the least square method based on the electron diffraction intensity data. The $X$-ray diffraction intensities from an elongated PPX specimen of the $\beta$-form were measured to analyse the 3-dimensional crystal structure. The result of the refinement using $x$-ray equatorial reflections was very similar to that by electron diffraction. Therefore the 3-dimensional analysis by the least square method was carried out with the constraint that the ab-plane projection should have the symmetry of p6. The 3-dimensional space group of the resulting structure is P3. Our result was confirmed by comparing the electron microscopic image with computer-simulated images. 


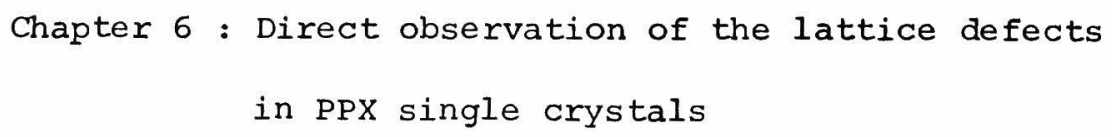

Lattice defects (edge dislocation) in PPX single crystals were resolved by high resolution electron microscopy. The edge dislocation observed in a $\beta$-form single crystal was discussed in terms of 5 types of dislocations predicted to exist in polymer crystals. The appearance of the edge dislocation in a $B$-form single crystal is well represented by the bubble model. The dislocation should be the partial one, with the magnitude of the Burgers vector being one fourth of the lattice constant. 
List of publications

1) "Construction of Image Processing Systems and Application to the Random Noise Removal in Electron Micrographs", Bull.Inst. Chem.Res., Kyoto Univ., 55, 237-247 (1977).

2) "Resolution of Individual Chains Composing Polymer Single Crystals with the High resolution Electron Microscope", Polymer Preprints, Japan, 29, 555 (1980).

3) "An Application of High Resolution Electron Micrographs to Crystal structure Analysis, --- Poly-p-Xylylene Crystal --- ", Polymer Preprints, Japan, 29, 556 (1980).

4) "Resolution of Individual Chains Composing Polymer Single Crystals with the High resolution Electron Microscope", Polymer Preprints, Japan, 29, 2111-2114(1980). 


\section{Acknowledgments}

The present study was carried out at the Laboratory of Polymer Crystals, the Institute for Chemical Research, Kyoto University, from 1975 to 1980 .

The author wishes to express his sincere gratitude to Professor Ken-ichi Katayama for his constant guidance, encouragement in the course of this study, and detailed criticism on the manuscript.

He also wishes to express his thanks to Emeritus Professor Keinosuke Kobayashi for his introduction to high resolution electron microscopy and his continuous encouragement.

Thanks are much due to Dr. Akiyoshi Kawaguchi, Mr. Seiji Isoda and Mr. Masayoshi O'hara for their kind collaboration throughout the study .

Thanks are also due to Dr. Kanji Kajiwara for his critical reading of the manuscript, and Mr. Shozo Murakami for his kind assistance in the preparation of the manuscript.

The author would like to acknowledge Professor Natsu Uyeda and Mr. Yoshinori Fujiyoshi for their permission to use their electron micrograph of vanadium oxide, and Dr. Kazuo Ishizuka for his permission to use his computer-program for kinematical image-simulation.

He is grateful to Tomoe Engineering Co. Ltd. for the supply of a PPX specimen ( Parylene $N$ ). 


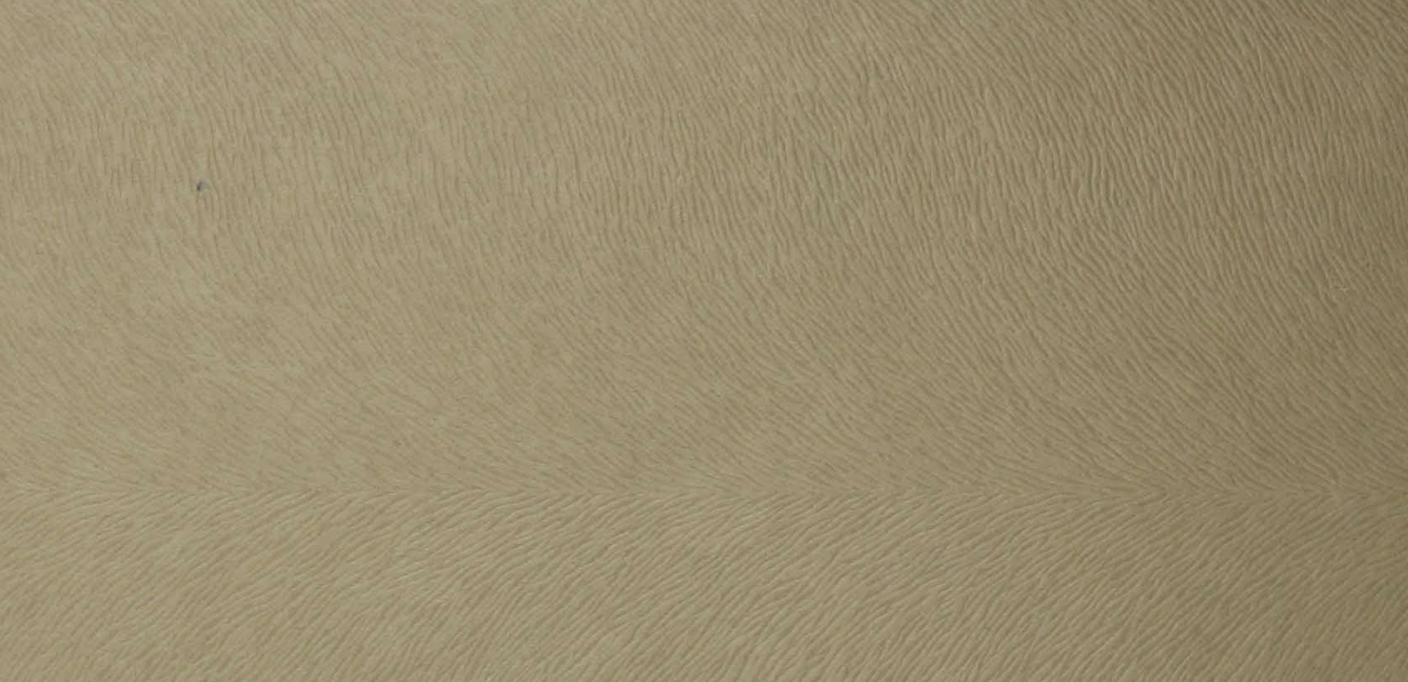

-

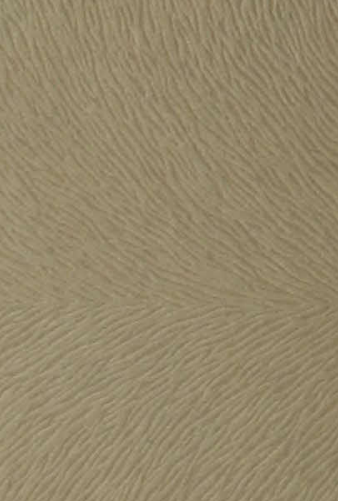
Hem

H. (c)

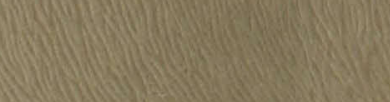
Halm

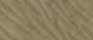

Whind

NAS N N N N (20)

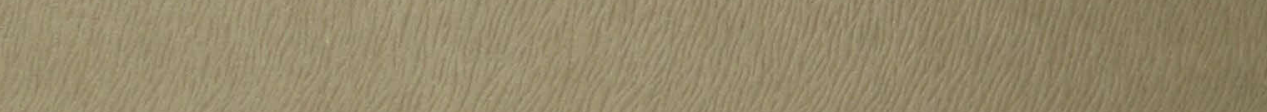
Q10

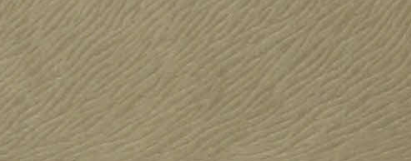
(1)

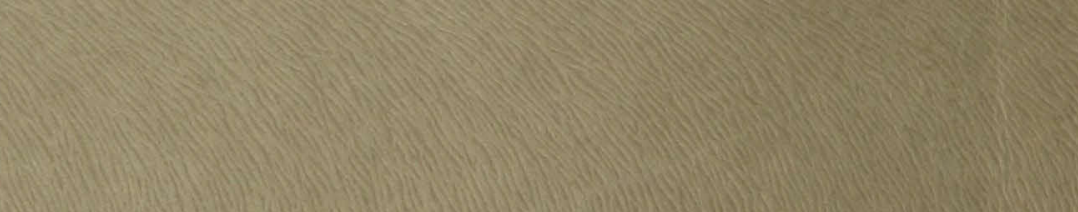

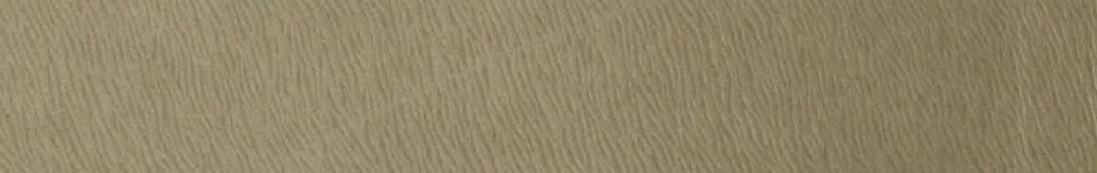
Whathom

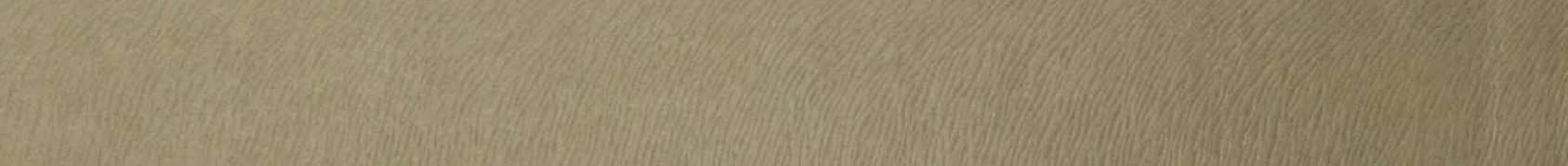
SW (2) 